\title{
Mannich Curcuminoids as Potent Anticancer Agents
}

Márió Gyuris', László Hackler Jr. ${ }^{1}$, Lajos I. Nagy ${ }^{1}$, Róbert Alföldi ${ }^{1}$, Eszter Rédei ${ }^{1}$, Annamária Marton ${ }^{2}$, Tibor Vellai ${ }^{3}$, Nóra Faragó ${ }^{1}$, Béla Ózsvári ${ }^{1}$, Anasztázia Hetényi ${ }^{4}$, Gábor K. Tóth ${ }^{4}$, Péter Sipos ${ }^{5}$, Iván Kanizsai id ${ }^{1}$, and László G. Puskás ${ }^{1 *}$

1 AVIDIN Ltd., Szeged, Hungary

2 Institute of Biochemistry, Biological Research Centre, Hungarian Academy of Sciences, Szeged, Hungary

${ }^{3}$ Faculty of General Medicine, Department of Medical Chemistry, University of Szeged, Szeged, Hungary

${ }^{4}$ Department of Genetics, Eötvös Loránd University, Budapest, Hungary

${ }^{5}$ Faculty of Pharmacy, Department of Pharmaceutical Technology, University of Szeged, Szeged, Hungary

A series of novel curcuminoids were synthesised for the first time via a Mannich-3CR/organocatalysed Claisen-Schmidt condensation sequence. Structure-activity relationship (SAR) studies were performed by applying viability assays and holographic microscopic imaging to these curcumin analogues for anti-proliferative activity against A549 and H1975 lung adenocarcinoma cells. The TNF $\alpha$-induced $\mathrm{NF}-\kappa \mathrm{B}$ inhibition and autophagy induction effects correlated strongly with the cytotoxic potential of the analogues. Significant inhibition of tumour growth was observed when the most potent analogue $\mathbf{4 4}$ was added in liposomes at one-sixth of the maximally tolerated dose in the A549 xenograft model. The novel spectrum of activity of these Mannich curcuminoids warrants further preclinical investigations.

Keywords: Anticancer / Autophagy / Curcuminoids / Mannich / NF-кB inhibition

Received: January 5, 2017; Revised: April 25, 2017; Accepted: April 25, 2017

DOI 10.1002/ardp.201700005

Additional supporting information may be found in the online version of this article at the publisher's web-site.

\section{Introduction}

Since cancer arises via multiple pathological or signalling pathways, certain natural compounds or their derivatives have the potential to be developed into optimum pharmaceuticals for cancer because of their ability to modulate multiple pathways. Numerous findings have demonstrated the relevance of herbal medicines for cancer therapy and prevention, including dietary phytochemicals among others [1]. Recent attention has focused on curcumin, also known as diferuloylmethane, a polyphenolic, yellow pigment found in the rhizome of turmeric (Curcuma longa). Curcumin has been shown to possess anti-oxidant, anti-inflammatory,

Correspondence: Dr. Iván Kanizsai, AVIDIN Ltd., Alsó kikötő sor 11, Szeged H-6726, Hungary.

E-mail: i.kanizsai@avidinbiotech.com

Fax: +3662202108 anti-angiogenic and anti-proliferative properties [1-3]. This broad spectrum of activity has been attributed to its ability to affect multiple targets, including transcription factors, growth factors, kinases, inflammatory cytokines, adhesion molecules, apoptosis-related proteins [4] and signalling pathways involving NF-kB, Akt, MAPK, Wnt, Notch and p53 [5-7]. One of the main targets of curcumin is the NF-кB cell signalling pathway [8]. Curcumin directly inhibits IKK and the $26 \mathrm{~S}$ proteasome $[9,10]$ to block NF-кB activation. Several studies have revealed that curcumin can induce death of cells that are resistant to apoptosis [11, 12], and treatment with curcumin has been reported to induce autophagic cell death in malignant cells [13-26].

Although numerous preclinical and clinical studies have demonstrated the biological potential of curcumin to treat

\footnotetext{
*Additional correspondence: Dr. László G. Puskás, E-mail: laszlo@avidinbiotech.com
} 
cancer patients, either alone or in combination with already existing therapeutic regimens [2], the clinical application of curcumin has been limited by its low potency and poor pharmacokinetics $[17,18]$. Therefore, in order to improve its activity and pharmaceutical profile, several structurally related compounds have recently been synthesised and evaluated as anticancer agents [19-32].

Different synthetic strategies have been reported to expand the molecular diversity, from side-chain and diketone transformations to alkyl and alkenyl functionalisations on C-4. Selected reports, including structure-activity relationship (SAR) studies on C-4-modified curcumin analogues 2-4 have disclosed significantly higher cytotoxic values than that of curcumin itself (1, Fig. 1) [30-32]. Structural modification on the central carbon through formation of a new $\mathrm{C}-\mathrm{C}$ double bond led to analogue 2, which displays higher activity against the growth of human lung adenocarcinoma cells $\mathrm{H} 1944$ and $\mathrm{A} 549$, with submicromolar $\mathrm{GI}_{50}$ values [30]. The 4-benzylated derivative 3 exhibits notable growth suppression of SW480 and other human colon cancer cells [31]. Furthermore, Michael addition to ethyl propiolate gave access to derivative 4, which exhibits low micromolar growth-inhibitory effects against MCF-7 and SKBR3 breast cancer cell lines [32].

The present report describes the synthesis of novel Mannich-type curcumin species as a new class of potential anticancer agents. These curcumin analogues were screened for their cytotoxicity in in vitro assays and for their NF$\kappa \mathrm{B}$-inhibitory activities. In vivo test results on a selected compound in a human lung cancer/SCID mouse tumour xenograft model are presented.

\section{Results and discussion}

\section{Chemistry}

In order to generate more potent curcumin analogues with favourable medicinal properties, we exploited the potential of multicomponent reactions. Precursors $\mathbf{5}$ were synthesized via a Mannich-type one-pot three-component assembly of pentane-2,4-dione, an aromatic aldehyde and an alkyl or alkenyl amide [33]. By a slight modification of the reported procedure, the multicomponent Mannich transformations were carried out in the presence of $\mathrm{TMSCl}$, affording $\mathrm{N}$-(2-acetyl-3-oxo-1-phenylbutyl)amide derivatives $\mathbf{5}$ in yields of $22-81 \%$ (Scheme 1 ).

In contrast with literature data, aromatic aldehydes bearing electron-donating groups did not undergo reaction under the conditions applied. For further variability, the Mannich intermediate with $\mathrm{R}_{1}=\mathrm{Ph}, \mathrm{R}_{2}=$ ethylene was subjected to Heck coupling, which resulted in $\mathrm{N}$-(2-acetyl-3-oxo-1-phenylbutyl)cinnamamide 6 . The curcumin backbone was constructed through a morpholinium chloroacetate-catalysed double aldol (Claisen-Schmidt) condensation (Scheme 1). To the best of our knowledge, this was the first utilization of morpholinium chloroacetate as an effective organocatalyst in synthetic transformations [34]. It should be noted that by the application of $n$-butyl amine (Pabon-method) or piperidine in<smiles>COc1cc(/C=C/C(=O)/C=C/c2ccc(/C=C/C(=O)CC(=O)/C=C/c3ccc(O)c(OC)c3)cc2OC)ccc1O</smiles><smiles>COc1ccc(/C=C/C(=O)C(=Cc2ccc(F)cc2)C(=O)/C=C/c2ccc(OC)c(OC)c2)cc1OC</smiles>

A549: $\mathrm{GI}_{50}=0.7 \mu \mathrm{M}$ $\mathrm{H} 1944: \mathrm{Gl}_{50}=0.28 \mu \mathrm{M}$<smiles>COc1cc(/C=C/C(=O)C(Cc2ccc(O)c(OC)c2)C(=O)/C=C/c2ccc(O)c(OC)c2)ccc1O</smiles>

$\mathrm{KM} 12: \mathrm{IC}_{50}=3.97 \mu \mathrm{M}$ SW480: $I \mathrm{C}_{50}=4.78 \mu \mathrm{M}$<smiles>CCOC(=O)/C=C/C(C(=O)/C=C/c1ccc(OC)c(OC)c1)=C(O)/C=C/c1ccc(OC)c(OC)c1</smiles>

Figure 1. Structures of curcumin $\mathbf{1}$ and compounds 2-4. 


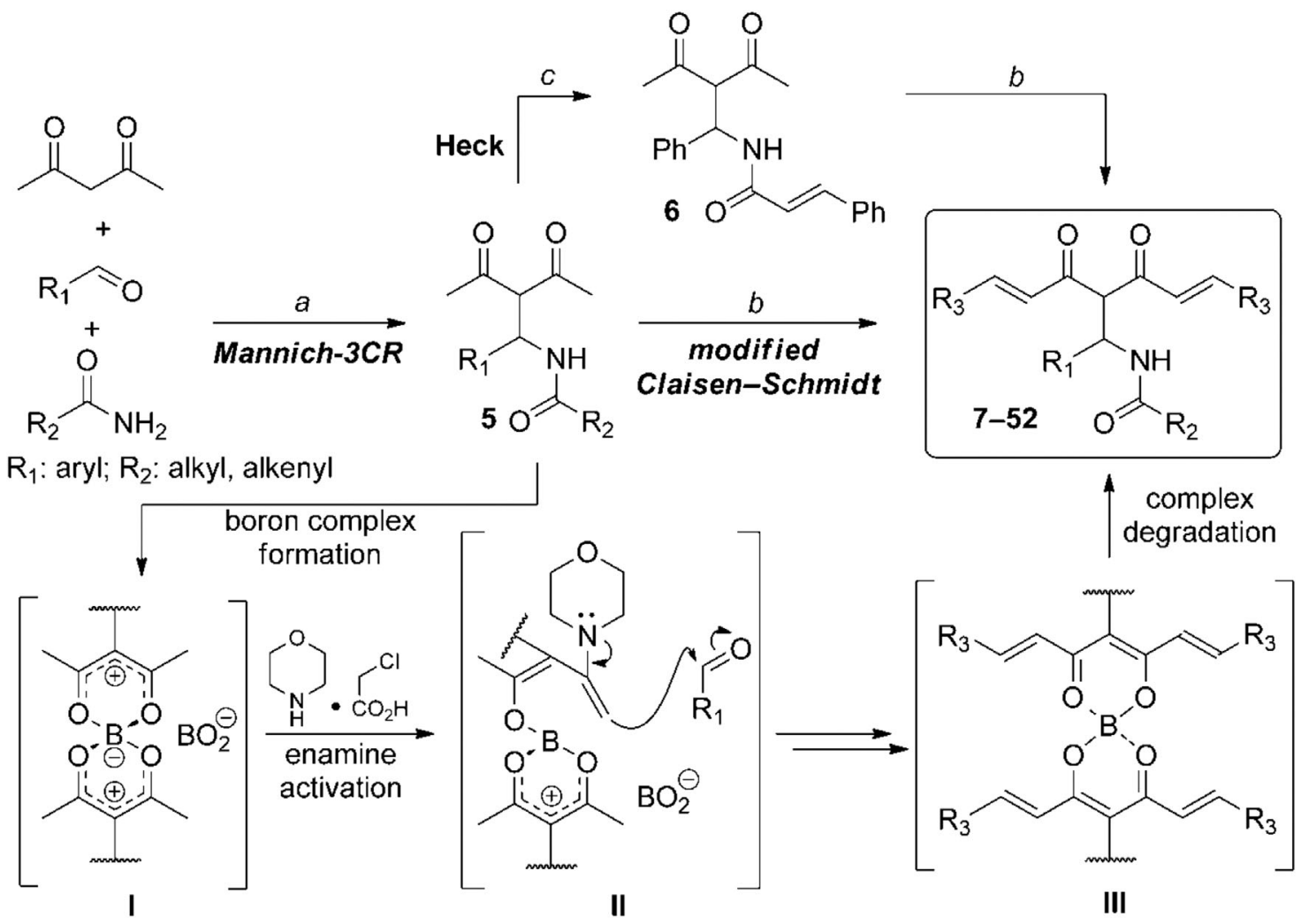

Scheme 1. Reagents and conditions: (a) $\mathrm{TMSCl}^{\mathrm{Et}} \mathrm{E}_{2} \mathrm{O} / \mathrm{MeCN}(1: 1), \mathrm{rt}, 12 \mathrm{~h} ; 22-81 \%$; (b) $\mathrm{B}_{2} \mathrm{O}_{3}, \mathrm{dry} \mathrm{DMF}, 75^{\circ} \mathrm{C}, 30 \mathrm{~min}, \mathrm{R}_{3} \mathrm{CHO}, \mathrm{B}(\mathrm{OBu})_{3}$, $75^{\circ} \mathrm{C}, 5 \mathrm{~min}$, morpholinium chloroacetate, $75^{\circ} \mathrm{C}, 4 \mathrm{~h}, 5 \%$ aqueous $\mathrm{AcOH}$ solution, $75^{\circ} \mathrm{C}, 1 \mathrm{~h} ; 40-75 \%$; (c) iodobenzene, $\mathrm{Pd}(\mathrm{OAc})_{2}, \mathrm{PPh}$, TEA, toluene, reflux, $2 \mathrm{~h} ; 71 \%$.

Claisen-Schmidt condensations, no reaction or complex reaction mixtures were observed.

The proposed mechanism includes the in situ formation of an intermediate spirocyclic boroncomplex I and its subsequent ring opening and closure, involving secondary amine saltcatalysed multiple aldol condensation via enamine activation, permitting access to a rosocyanine-type transition state III. Finally, acid-mediated degradation yielded curcuminoids 7-52 in isolated yields of $40-75 \%$.

However, the application of o-substituted benzaldehydes led to complex reaction mixtures during the modified ClaisenSchmidt condensation, presumably due to both steric and electronic effects.

With this synthetic approach, a 46-membered curcuminoid library was generated and characterized. Scale-up trials on $\mathbf{4 4}$ revealed that the protocol proceeds on a multigram scale without any alteration in terms of yield, purity or reaction time (see Experimental section). NMR analysis of 7-52 confirmed the exclusive presence of the 1,3-diketo form in DMSO- $d_{6}$; keto-enol tautomerism could not be detected, in contrast with the reported tautomeric equilibrium of curcumin (1) [35].

\section{Biological results and SAR}

In vitro cytotoxicity assays were carried out with two different lung cancer cell lines (A549 and H1975) in order to evaluate the anticancer activities of the new derivatives 7-52. A total of $95 \%$ of all the toxicity measurements exhibited less than $10 \%$ variation. For comparison, concentration-response curves were obtained to determine $\mathrm{GI}_{50}$ for each compound, using curcumin (1) and reference compound 2 [35] as positive controls. The $\mathrm{Gl}_{50}$ values obtained after an exposure time of $72 \mathrm{~h}$ are reported in Tables $1-5$.

The synthesised analogues were categorised into three main groups on the basis of their structural similarity and the modifications of $R_{1}, R_{2}$ and $R_{3}$ : acetamide analogues 7-36 (group 1 subclasses 1-3), acrylamide derivatives 37-48 (group 2) and cinnamides 49-52 (group 3).

Acetamide derivatives - subclass 1

The levels of in vitro anticancer efficiency of 7-19 were examined and the results were compared with those of 1 and 2. In this subclass, $R_{1}$ varied with fixed $R_{2}=M e(-N H A c)$ and $\mathrm{R}_{3}=3,4-\mathrm{MeOC}_{6} \mathrm{H}_{3}$ units (Table 1 ). With two exceptions (18 and 19), the substituted phenyl derivatives 8-17 exerted 
Table 1. Acetamide derivatives 7-19 (subclass 1) versus A549 and H1975 cancer cell lines.

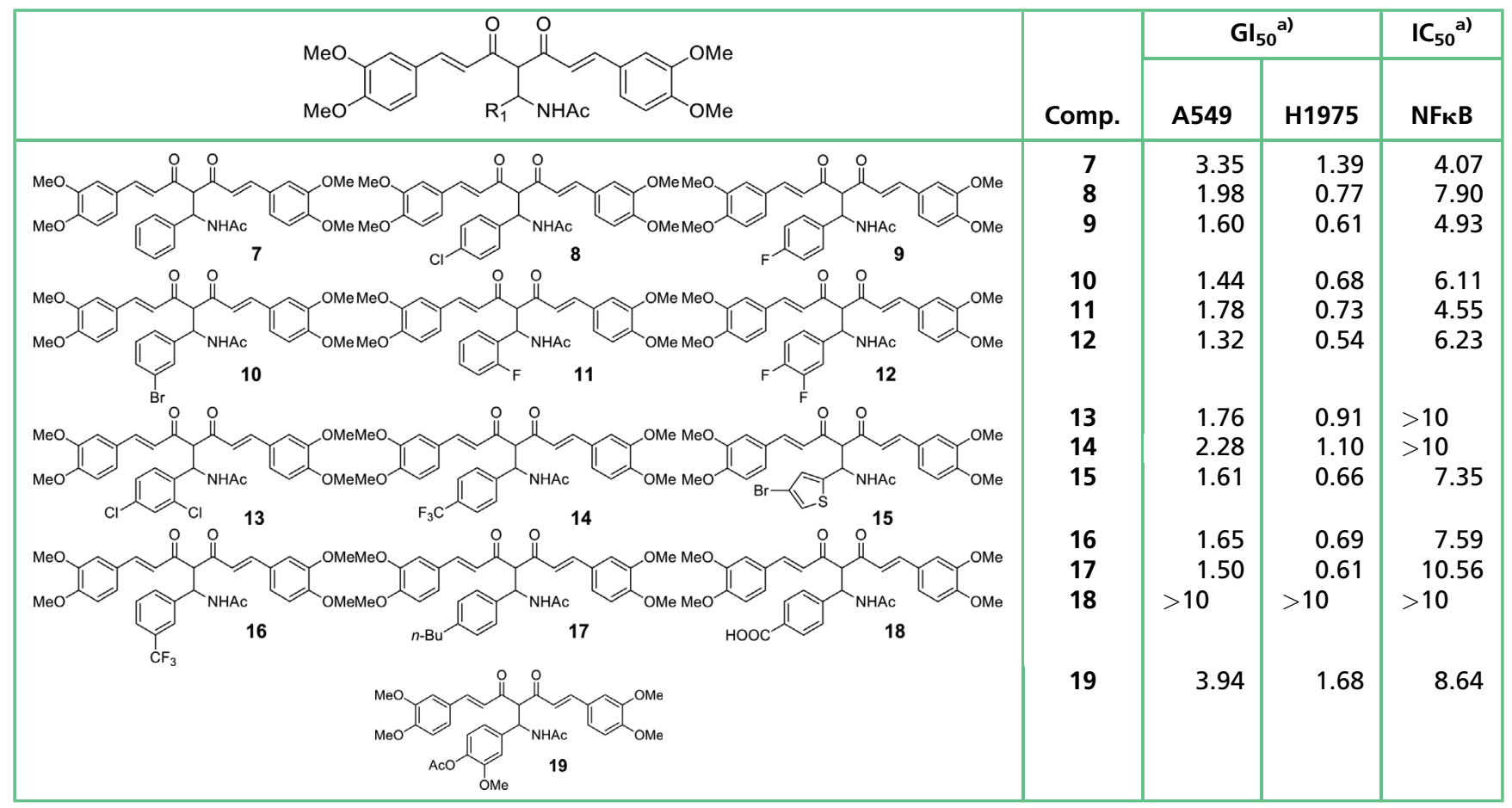

a) Concentration in $\mu \mathrm{M}$; reference $\mathrm{Gl}_{50}$ and $\mathrm{IC}_{50}$ values for comparison: curcumin 121.37 (A549), 25.80 (H1975), 104.10 (NF- $\left.\mathrm{KB}\right)$ and compound 21.21 (A549), 0.63 (H1975), 9.68 (NF-кB), in $\mu \mathrm{M}$.

higher in vitro cytotoxic activities on A549 and H1975 human lung adenocarcinoma cells than those of the unsubstituted derivative $7\left(\mathrm{R}_{1}=\mathrm{Ph}\right)$ or the parent compound 1 . Compounds 9, 12 and 17 displayed levels of efficacy similar to those of $\mathbf{2}$ on both cell lines. Introduction of a para- $\mathrm{CF}_{3}$ group (14) resulted in a lower activity than those of either the regioisomer meta$\mathrm{CF}_{3}$ analogue $\mathbf{1 6}$ or any mono- or di-halogenated compound. A para-COOH group (18) completely abolished the cytotoxic

Table 2. Acetamide derivatives 20-27 (subclass 2) versus A549 and H1975 cancer cell lines.

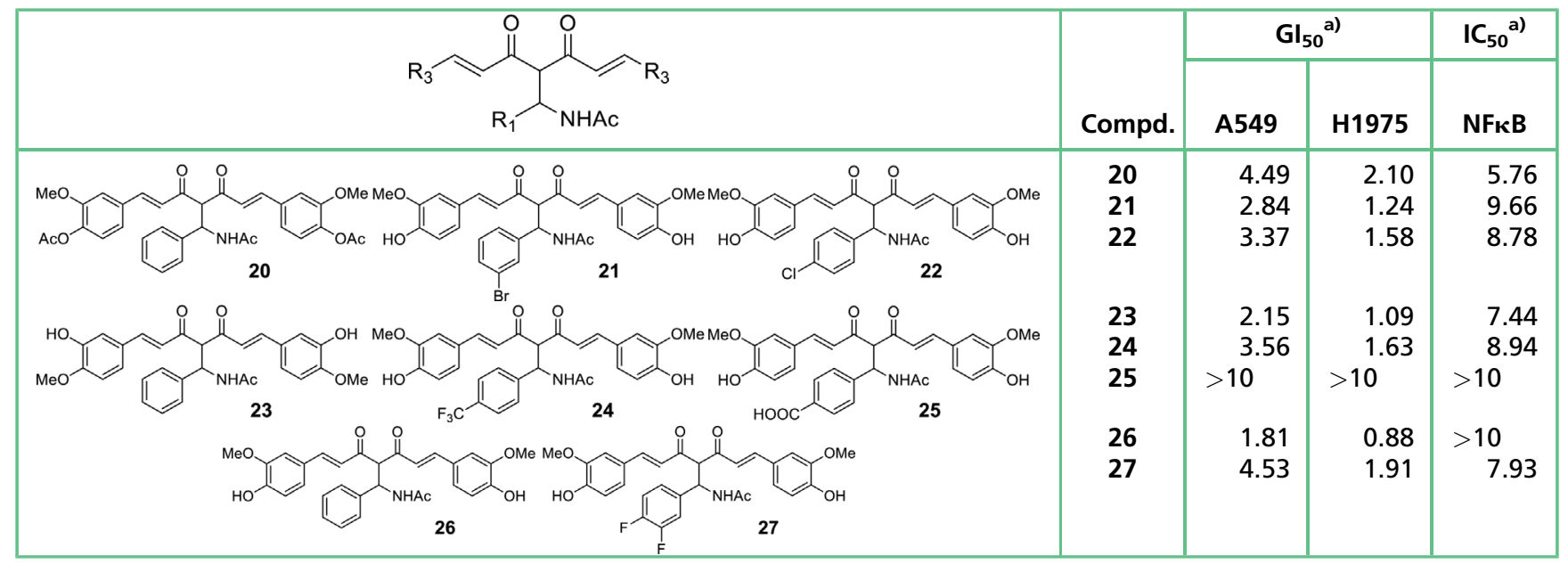

a) Concentration in $\mu \mathrm{M}$; reference $\mathrm{Gl}_{50}$ and $\mathrm{IC}_{50}$ values for comparison: curcumin $121.37(\mathrm{~A} 549), 25.80(\mathrm{H} 1975), 104.10(\mathrm{NF}-\kappa \mathrm{B})$ and compound 21.21 (A549), 0.63 (H1975), 9.68 (NF-кB), in $\mu \mathrm{M}$. 
Table 3. Acetamide derivatives 28-36 (subclass 3) versus A549 and H1975 cancer cell lines.

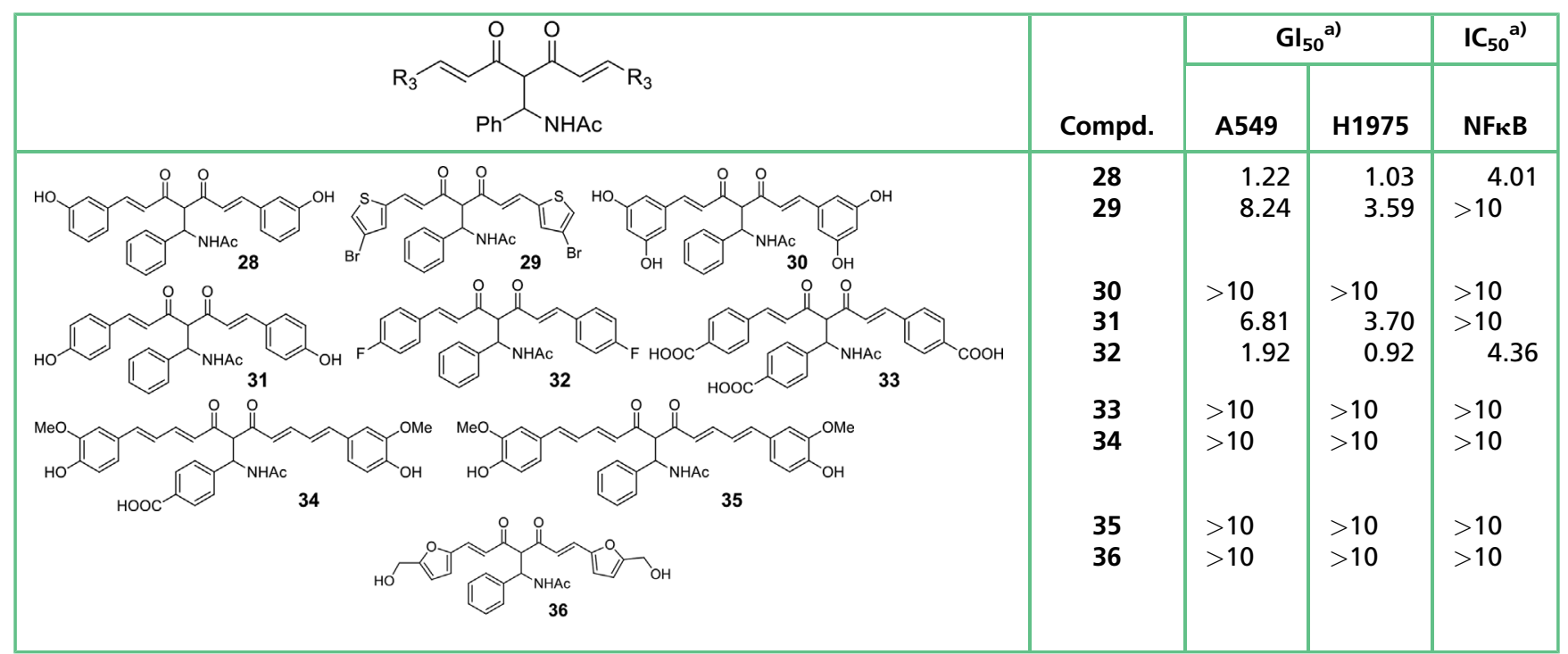

a) Concentration in $\mu \mathrm{M}$, reference $\mathrm{Gl}_{50}$ and $\mathrm{IC}_{50}$ values for comparison: curcumin 121.37 (A549), 25.80 (H1975), 104.10 (NF- $\mathrm{B}$ ) and compound 21.21 (A549), 0.63 (H1975), 9.68 (NF-кB), in $\mu \mathrm{M}$.

Table 4. Acrylamide derivatives 37-48 versus A549 and H1975 cancer cell lines.

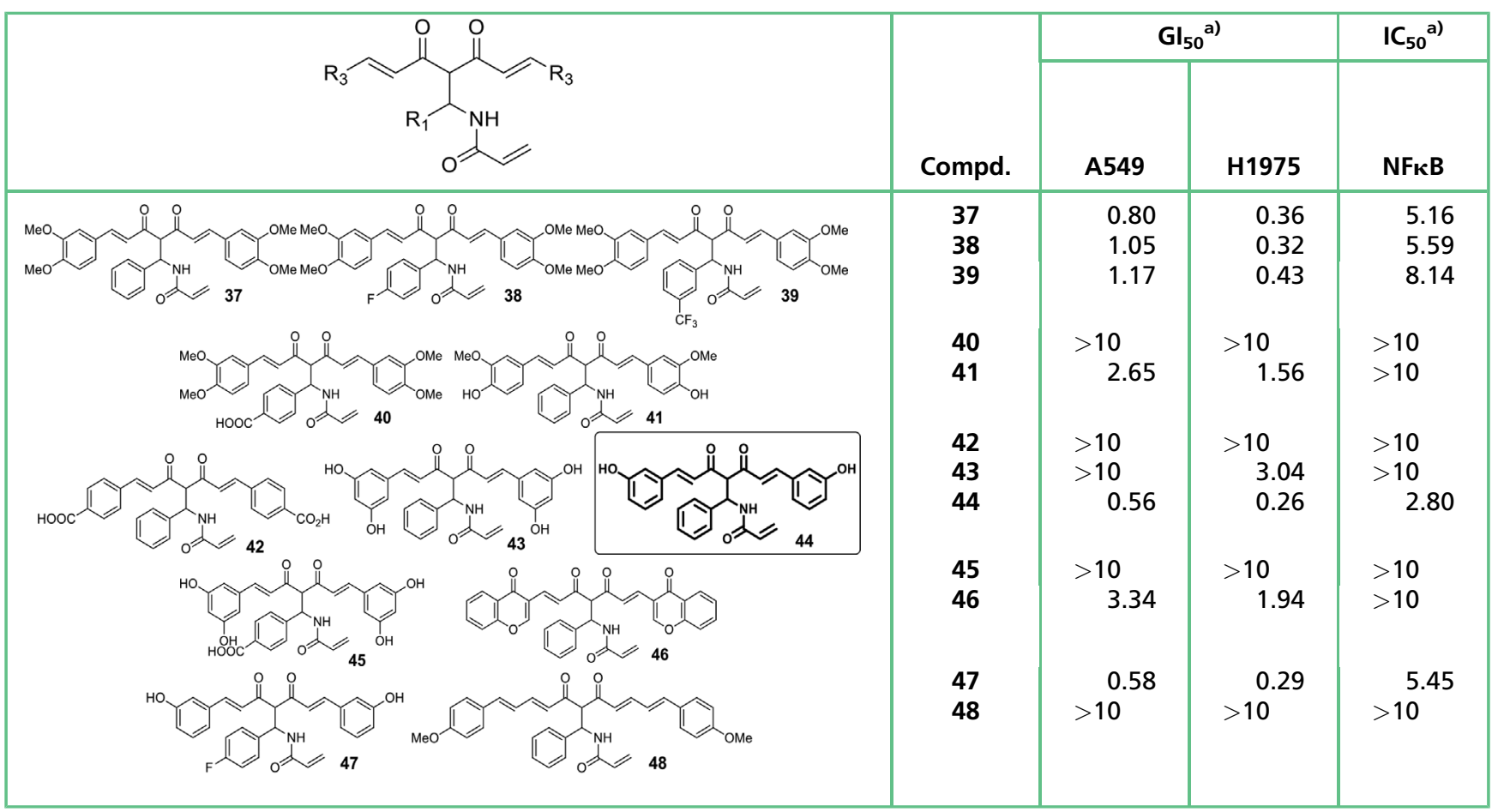

a) Concentration in $\mu \mathrm{M}$; reference $\mathrm{Gl}_{50}$ and $\mathrm{IC}_{50}$ values for comparison: curcumin $121.37(\mathrm{~A} 549), 25.80(\mathrm{H} 1975), 104.10(\mathrm{NF}-\mathrm{\kappa} \mathrm{B})$ and compound 21.21 (A549), 0.63 (H1975), 9.68 (NF-кB), in $\mu \mathrm{M}$. 
Table 5. Cinnamide derivatives 49-52 versus A549 and H1975 cancer cell lines.

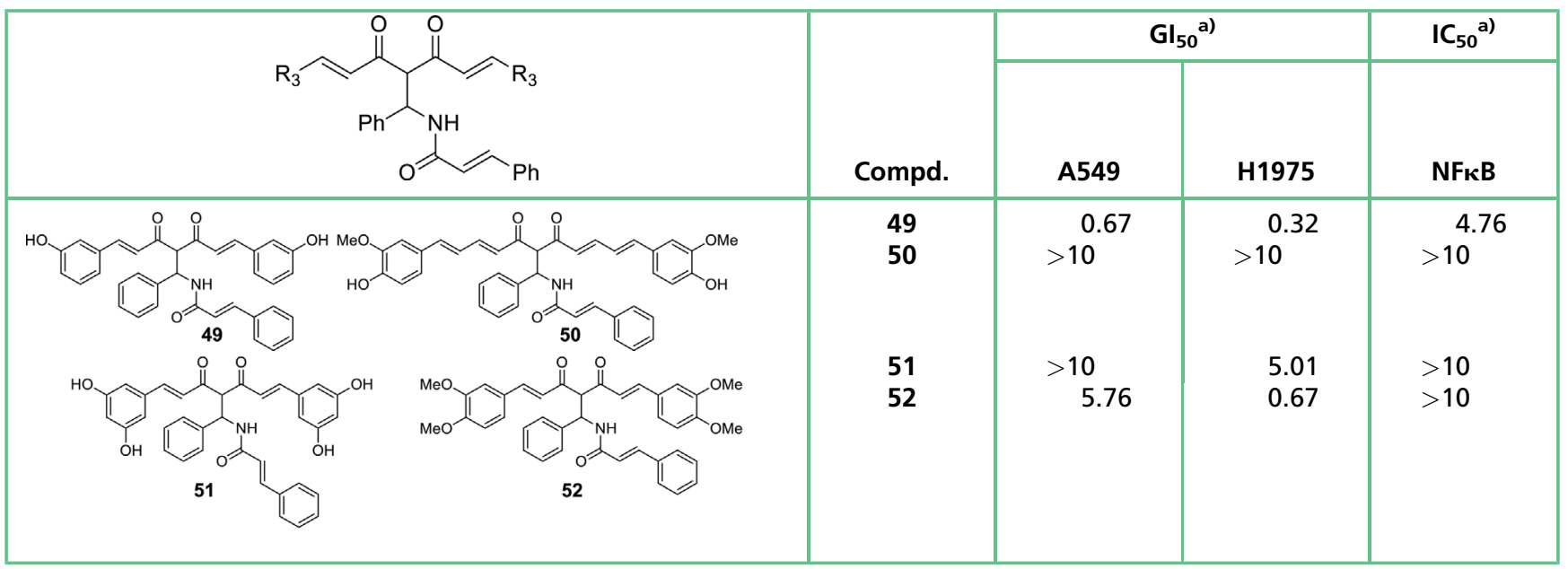

a) Concentration in $\mu \mathrm{M}$; reference $\mathrm{Gl}_{50}$ and $\mathrm{IC}_{50}$ values for comparison: curcumin 121.37 (A549), 25.80 (H1975), 104.10 (NF-кB) and compound 21.21 (A549), 0.63 (H1975), 9.68 (NF-kB), in $\mu \mathrm{M}$.

activity. The in vitro NF-кB inhibition tests indicated that, apart from 17, the compounds in this subclass exerted stronger action than 2. Compounds 7-17 gave rise to a one order of magnitude higher NF-кB inhibition than that of $\mathbf{1}$.

\section{Acetamide derivatives - subclass 2}

In this subset, $R_{1}$ and $R_{3}$ varied, while $R_{2}$ was fixed $\left(R_{2}=M e\right.$; -NHAc) (Table 2). Analogues 20-27 demonstrated a lower level of cytotoxic activity as compared with 8-17, except for the activities of $23\left(\mathrm{R}_{1}=\mathrm{Ph}, \mathrm{R}_{3}=3-\mathrm{HO}-4-\mathrm{MeOC}_{6} \mathrm{H}_{3}\right)$ and 26 $\left(\mathrm{R}_{1}=\mathrm{Ph}, \mathrm{R}_{3}=4-\mathrm{HO}-3-\mathrm{MeOC}_{6} \mathrm{H}_{3}\right)$. Similarly as in subclass 1 , the introduction of a $p-\mathrm{HOOCC}_{6} \mathrm{H}_{4}$ group as $\mathrm{R}_{1}$ (derivative 25 ) led to the complete loss of anticancer activity. It should be noted that the activity was mainly influenced by the nature of $R_{1}$ : the best results were obtained when it was an unsubstituted phenyl function (23 and 26) or a hydroxy group. Modification of $R_{3}$ to the acylated form of isovanillin diminished the cytotoxic potential (compound 20).

\section{Acetamide derivatives - subclass 3}

In analogues 28-36, $\mathrm{R}_{3}$ varied, while $\mathrm{R}_{1}$ was $\mathrm{Ph}$ or $4-\mathrm{HOOCC}_{6} \mathrm{H}_{4}$ (Table 3 ). Compounds containing a heteroaromatic $R_{3}$, e.g. 3bromothiophene (29) exhibited relatively low potency, while furan derivative $\mathbf{3 6}$ was inactive in the tested range, similarly to the compounds containing a 3,5- $\mathrm{HOC}_{6} \mathrm{H}_{3}(30)$ or a 2-MeO-4vinyl- $\mathrm{C}_{6} \mathrm{H}_{3}$ side-chain (34 and 35). Appreciable activity against the tested lung cancer cells was achieved when $\mathrm{R}_{3}$ was either $3-\mathrm{HOC}_{6} \mathrm{H}_{4}$ or $4-\mathrm{FC}_{6} \mathrm{H}_{4}$ (28 and 32). Conversely, the cytotoxicity was completely lost when a carboxyl group was present, regardless of its position (33 and 34 ).

\section{Acrylamide derivatives}

The replacement of acetyl by acryloyl as group $R_{2}$ resulted in significantly improved cytotoxic activity against both cell lines, with nanomolar $\mathrm{Gl}_{50}$ values (Table 4, 37, 44 and 47). Moreover, derivative 44 , in which $\mathrm{R}_{1}=\mathrm{Ph}$ and $\mathrm{R}_{3}=3-\mathrm{HOC}_{6} \mathrm{H}_{4}$ exerted excellent NF-kB inhibition also and was therefore assigned as lead compound for further investigations.

\section{Cinnamide derivatives}

Intermediate 6 was synthesized via the Heck reaction and was converted to 49-52 (Table 5). Although the in vitro activity of 49 was much more pronounced than those of $\mathbf{5 0 - 5 2}$, it was not significantly different from that of lead compound $\mathbf{4 4}$. Interestingly, $\mathbf{3 7}$ (Table 4) was significantly less active than $\mathbf{5 1 .}$ Replacement of the acrylamide framework by cinnamide generally leads to a decrease in anticancer activity.

The overall in vitro results allowed the following conclusions concerning the SAR: When $\mathrm{R}_{1}=\mathrm{Ph}, 4-\mathrm{FC}_{6} \mathrm{H}_{4}$ or $3-\mathrm{F}_{3} \mathrm{CC}_{6} \mathrm{H}_{4}, \mathrm{R}_{2}=$ vinyl and/or $\mathrm{R}_{3}=3-\mathrm{HOC}_{6} \mathrm{H}_{4}$ or $3,4-\mathrm{MeOC}_{6} \mathrm{H}_{3}$, we observed noteworthy potency in terms of in vitro cytotoxicity and NF-кB inhibition. Interestingly, introduction of a carboxyl functionality at any position completely abolished the cytotoxicity. It was not determined that this lack of activity was due to decreased activity at molecular targets or due to limited cell permeability of carboxyl group bearing compounds. Moreover, none of the derivatives with a 3,4- $-\mathrm{HOC}_{6} \mathrm{H}_{3}$ side-chain, regardless of the nature of $\mathrm{R}_{1}$ and $\mathrm{R}_{2}$, exhibited activity against lung carcinoma cell growth in the tested concentration range. The most active novel analogues displayed 40- to 100-fold better potency than 1 in these assays. While 1 exhibited $\mathrm{Gl}_{50}$ values of 21.4 and $25.8 \mu \mathrm{M}$ against A549 and H1975 cancer cell lines, respectively, and the previously described 2 exhibited improved anti-proliferation activities (1.2 and $0.56 \mu \mathrm{M})$, lead compound 44 exerted even better anticancer activity $\left(\mathrm{Gl}_{50}\right.$ values of 0.56 and $\left.0.26 \mu \mathrm{M}\right)$, and is probably the most potent synthetic curcumin derivative reported to date (Fig. 2). 

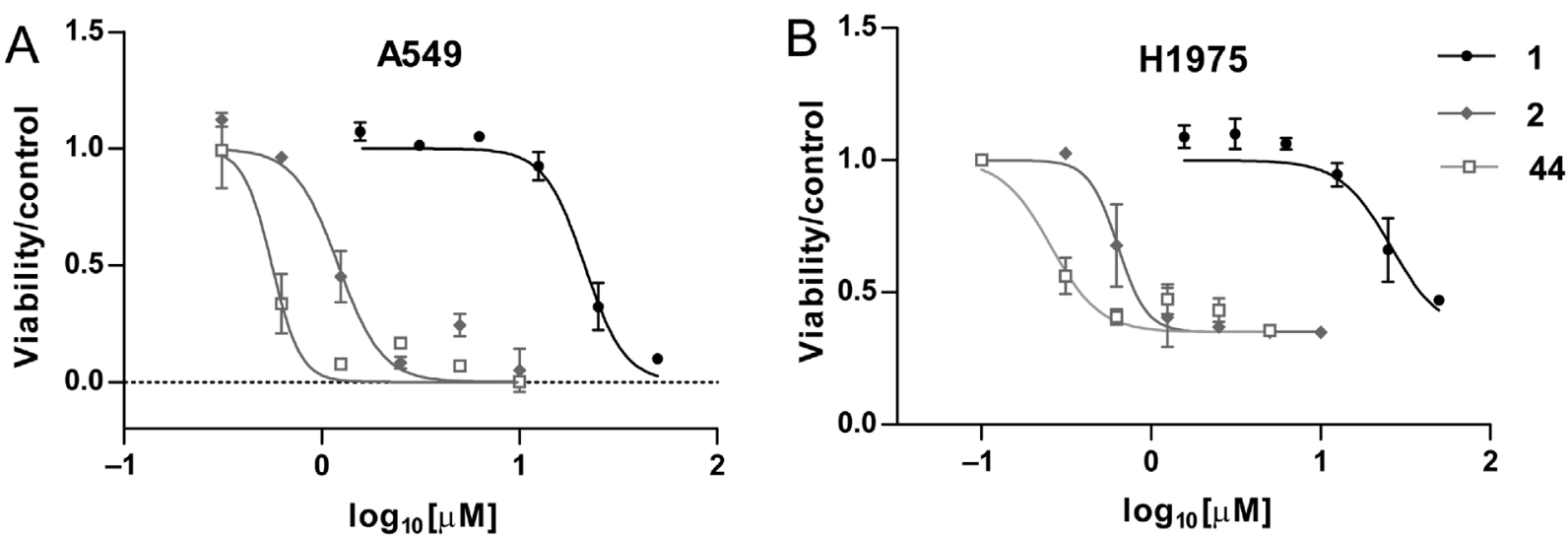

Figure 2. Effects on lung cancer cell viability of 1, 2 or 44, applied for $72 \mathrm{~h}$. Cell viability was assessed by the MTS method and expressed as a fraction of the vehicle control (DMSO). (A) Lung adenocarcinoma, A549. (B) Lung adenocarcinoma, H1975.

\section{Holographic cell analysis}

To visualise the cytotoxic effects of the most potent analogue 44, holographic microscopic analysis was performed. With this technology, morphological parameters of treated cells such as area, thickness and volume can be followed in a label-free way [36, 37]. A549 human lung adenocarcinoma cells were incubated with $0.5 \mu \mathrm{M} 44$ or $25 \mu \mathrm{M} 1$ as a control, and holographic images were taken before and $24 \mathrm{~h}$ after treatment. The morphological changes induced by the cytotoxic effects after $24 \mathrm{~h}$ are illustrated through holographic images in Fig. 3. A decrease in cell surface and an increase in average optical thickness were registered after treatment, as the affected cells started to show the typical profile of dying cells, changing to a spherical shape and becoming detached from the surface. Similar shifts in the parameters of area and thickness were observed after treatment with $\mathbf{1}$ or $\mathbf{4 4}$, but the effect of $\mathbf{4 4}$ was more pronounced than that of 1 , even at a 50 times lower concentration. The dynamics of the morphology change indicated that the effect of the treatment was already manifested after only $8 \mathrm{~h}$ of incubation in the case of $\mathbf{4 4}$, although with a slight shift in distribution (data not shown).

Inhibition of $T N F \alpha$ induced NF- $\kappa B$ activation by the new analogues

$\mathrm{NF}-\mathrm{KB}$ proteins influence the expression of genes involved in a large number of physiological processes, including immune response, cell survival, differentiation, and proliferation [38]. One of the main targets of curcumin is the NF- $\mathrm{kB}$ cell signalling pathway [8]. The correlation between the NF$\kappa \mathrm{B}$-inhibitory potential of different curcumin analogues and their cytotoxicity was reported recently [39].

A B16 cell line stably expressing an NF-KB luciferase reporter construct was used to screen the sythesised analogues for their NF-кB-inhibitory properties. Preincubation of cells with increasing concentrations of curcumin and its analogues dosedependently inhibited the TNF $\alpha$-induced NF-кB expression (while cell viability was not affected). $\mathbf{4 4}$ proved to be very potent, inhibiting NF- $\mathrm{kB}$ activation at micromolar concentration $\left(I C_{50}=2.8 \mu \mathrm{M}\right)$. This value is about 40 times lower than that of $1\left(\mathrm{IC}_{50}=104 \mu \mathrm{M}\right)$.

The data in Fig. 4 , where determined inhibitory $I_{50}$ values were compared with $\mathrm{Gl}_{50}$ values obtained in the cell toxicity assays, suggest an important correlation between the antiproliferative activity and the NF- $\mathrm{KB}$-inhibitory activity of the tested compounds. Active curcumin analogues behaved consistently in both assays, with $\mathbf{4 4}$ being the most potent in both. Analogues 30, 42 and 45, which did not exert antiproliferative activity, were also inactive in NF- $\mathrm{KB}$ activation inhibition. These results strongly support the notion that the NF-KB pathway is one of the major molecular targets for curcumin and its analogues.

\section{Unfolded protein response-related gene expression analysis}

Several factors are known to be responsible for the ER stressmediated transcriptional activation of HSPA5 (GRP78), including XBP-1 and activated ATF-4. ATF-4 is a cAMP response element-binding transcription factor that activates the transcription of genes involved in UPR and ER stress response [40]. Curcumin has been shown to induce apoptotic cell death initiated by the activation of the UPR signalling pathway [41]. UPR-related gene expression changes following treatment with the novel analogues were investigated. A549 cells were treated with the selected compounds at 1 and $5 \mu \mathrm{M}$, and harvested after $6 \mathrm{~h}$ of incubation. Table 6 lists the results of the QRT-PCR measurements. The selected inactive analogue 40 did not alter the expression of XBP-1 or DDIT3, whereas the active analogues $2,7,37,40$ and 41 increased the expression of both genes significantly, in a dose-dependent manner. 1 increased the expression of DDIT3 and HSPA5 only at $25 \mu \mathrm{M}$, but no induction was registered for the ATF4 and XBP1 genes. The expression of HSPA5 also displayed a significant, dose-dependent increase after treatment. 
A

B
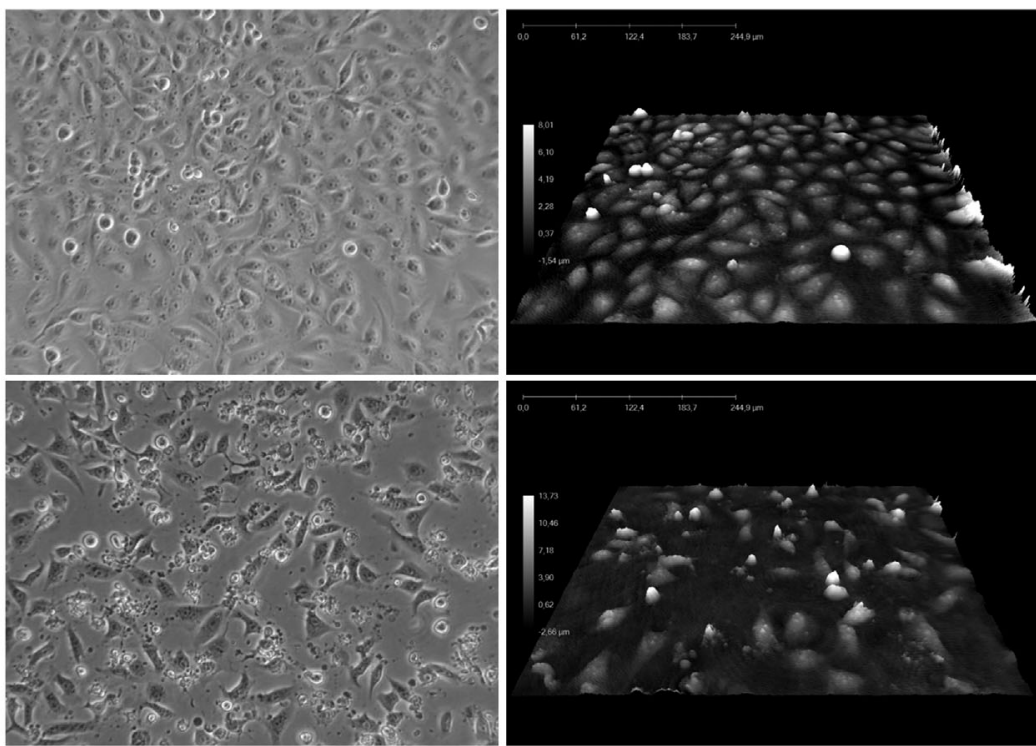

C

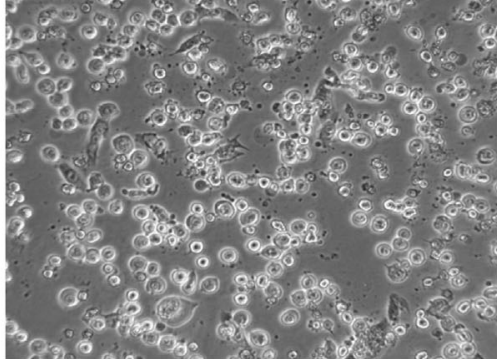

D

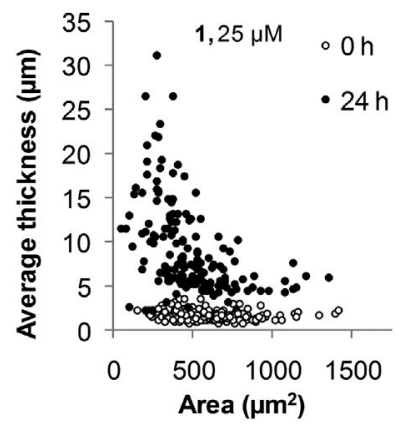

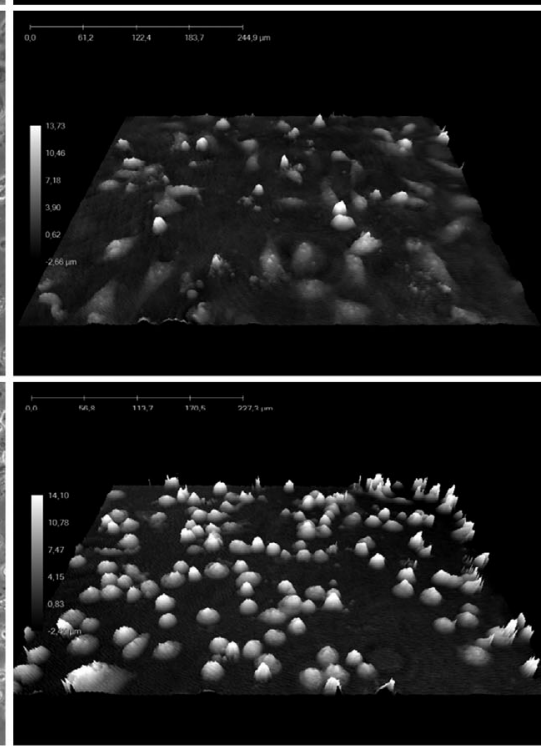

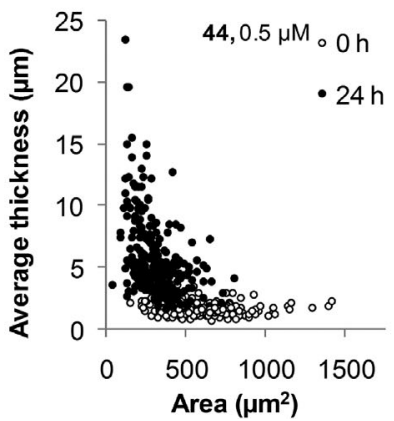

Figure 3. (A) Phase-contrast (left) and holographic image (right) of untreated A549 cells. (B) $25 \mu \mathrm{M}$ 1-treated cells at $24 \mathrm{~h}$ posttreatment. (C) $0.5 \mu \mathrm{M} 44$-treated cells at $24 \mathrm{~h}$ post-treatment. (D) Change in distribution of phenotypic parameters (cell area vs. average cell thickness) of 1-treated (left) and 44-treated (right) cells.
Effects of $\mathbf{4 4}$ on the autophagic machinery

It was earlier shown that bis-dehydroxycurcumin not only induces ER stress, but, as a downstream consequence, together with mitochondrial-dependent apoptosis, induces the autophagic machinery [42]. Recently, a TFEB activating novel curcumin analog was shown to promote autophagy [43]. We have investigated the effect of our most potent compound $\mathbf{4 4}$ on autophagy with three methodologies.

First, a cell-based assay was used to investigate the autophagic induction potential of $\mathbf{4 4}$. To show the delivery of autophagosomes to the lysosomes, an RFP-GFP (red fluorescent protein-green fluorescent protein)-tagged LC3B construct expressing HeLa cell line was used [44]. This experimental setup enabled us to discriminate early autophagic organelles (GFP-positive and mRFP-positive) from acidified autolysosomes (GFP-negative and mRFP-positive), due to quenching of the GFP signal exclusively inside acidic compartments [44]. After a $6 \mathrm{~h}$ incubation in the presence of 44 or controls (autophagy activator: rapamycin, autophagy inhibitor: bafilomycin), images were taken with a fluorescent confocal microscope in both red and green channels.

Figure 5 shows representative images of 44, rapamycin and bafilomycin treated cells. $\mathbf{4 4}$ treatment, similarly to rapamycin, resulted in marked increase of red puncta that represent acidic autolysosomes, suggesting an activated autophagic machinery. 


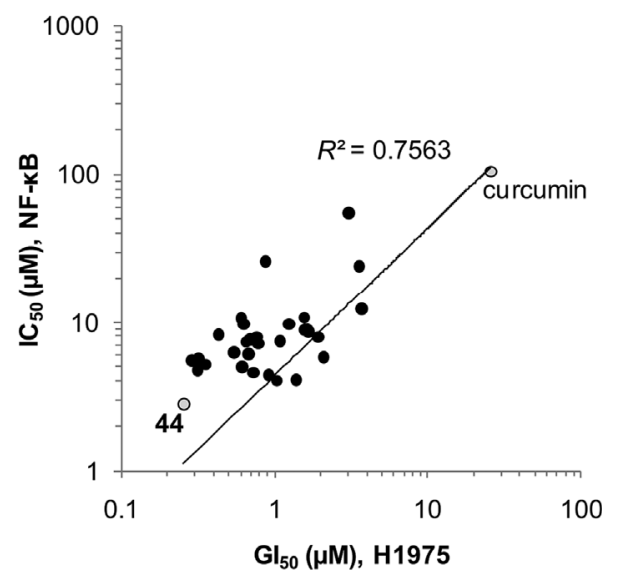

Figure 4. Correlation of $\mathrm{Gl}_{50}$ values obtained with $\mathrm{H} 1975$ cells and NF- $\mathrm{KB}$ induction inhibition $\mathrm{IC}_{50}$ values. $\mathrm{GI}_{50}$ and $\mathrm{IC}_{50}$ values are plotted for those analogs that were active in both measurements. A linear regression was fitted on the scatterplot.

Then, autophagy-related genes were selected and their expression was measured in A549 cells following treatment with 44 along with rapamycin. Gene expression was measured at 6,12 and $24 \mathrm{~h}$ after treatment. All tested genes showed time or dose-dependent activation by $\mathbf{4 4}$ (Fig. 6). Expression of MAP1LC3B a key molecule in autophagy was activated by $\mathbf{4 4}$ even after $6 \mathrm{~h}$, while rapamycin activated its expression only after $12 \mathrm{~h}$. Even the lowest applied concentration of $\mathbf{4 4}$ $(0.5 \mu \mathrm{M})$ activated the expression of BECN1, GABARAP and SQSTM1.

Thereafter, the expression of LC3B and p62 protein was measured by Western blot analysis in A549 cells following 44 treatment. 44 decreased the amount of LC3B-II while the cytosolic LC3B-I was still present suggesting an active autophagic process (Fig. 7B). Bafilomycin treatment inhibited autophagy evidenced by the accumulation of LC3B-II protein.

A considerable decrease in p62 protein level was detected only after $24 \mathrm{~h}$ in treated cells but not at 6 or $12 \mathrm{~h}$ (data not shown). Here only the $0.5 \mu \mathrm{M}$ concentration was tested for $\mathbf{4 4}$ since cells above this dose were showing massive signs of cell death preventing protein analysis. At $24 \mathrm{~h}$ the used positive control, rapamycin decreased significantly the level of p62. 44 treatment also resulted in a slight decrease of protein level (Fig. 7D).

\section{Liposome-encapsulated $\mathbf{4 4}$ reduced the size of human lung tumour xenografts}

The effects of the most potent analogue $\mathbf{4 4}$ were tested against subcutaneously implanted human lung cancer (A549) in the SCID mouse xenograft model. To circumvent bioavailability problems, we used a liposome delivery system for the encapsulation of $\mathbf{4 4}$ and intravenous administration. Liquid phase 44-containing liposomes were prepared by hydrating and redispersing a lipid film of CHOL/PC/DSPE-mPEG and 44 in PBS solution to a final concentration of $1 \mathrm{mg} / \mathrm{mL}$ of 44 . The droplet size, SSA (specific surface area) and PDI (polydispersity index) of the liposomes were measured by laser diffractometry $(n=5)$ by the wet method. The $d(0.1), d(0.5)$ as median and $d(0.9)$ droplet sizes, SSA and PDI were $75 \pm 19 \mathrm{~nm}$, $114 \pm 20 \mathrm{~nm}, \quad 178 \pm 24 \mathrm{~nm}, \quad 54.5 \mathrm{~m}^{2} / \mathrm{g}$ and $0.901 \pm 0.20$, respectively.

Administration of 44-containing liposomes, as an intravenous injection, was initiated on day 7 and subsequently applied three times a week for 4 weeks at one-sixth of the tolerated dose $(3 \mathrm{mg} / \mathrm{kg})$. A significant reduction in tumour

Table 6. Summary of QRT-PCR analyses on UPR-related gene expression.

\begin{tabular}{|c|c|c|c|c|c|c|c|c|c|c|}
\hline $\begin{array}{l}\mathrm{Gl}_{50} \\
(\mu \mathrm{M})\end{array}$ & Compd. & $\mu \mathrm{M}$ & HSPA5 & $p$-Value & ATF4 & $p$-Value & XBP1 & $p$-Value & DDIT3 & $p$-Value \\
\hline \multirow[t]{2}{*}{21.37} & 1 & 10 & 1.18 & 0.465 & 0.85 & 0.713 & 1.02 & 0.911 & 1.24 & 0.672 \\
\hline & & 25 & 4.27 & 0.002 & 1.24 & 0.667 & 1.28 & 0.177 & 4.96 & 0.002 \\
\hline \multirow[t]{2}{*}{1.214} & 2 & 1 & 4.14 & 0.001 & 3.81 & 0.020 & 2.60 & 0.005 & 19.52 & 0.001 \\
\hline & & 5 & 7.01 & $<0.001$ & 3.89 & 0.019 & 3.27 & 0.002 & 27.79 & 0.001 \\
\hline \multirow[t]{2}{*}{3.35} & 7 & 1 & 2.03 & 0.016 & 4.41 & 0.014 & 2.17 & 0.010 & 6.22 & 0.007 \\
\hline & & 5 & 6.45 & $<0.001$ & 3.73 & 0.021 & 4.17 & 0.001 & 89.68 & $<0.001$ \\
\hline \multirow[t]{2}{*}{0.8} & 37 & 1 & 2.30 & 0.009 & 2.79 & 0.045 & 2.16 & 0.011 & 17.11 & 0.001 \\
\hline & & 5 & 5.94 & 0.001 & 3.39 & 0.027 & 3.55 & 0.002 & 96.11 & $<0.001$ \\
\hline \multirow[t]{2}{*}{$>10$} & 40 & 1 & 2.30 & 0.009 & 1.05 & 0.891 & 1.40 & 0.121 & 0.71 & 0.388 \\
\hline & & 5 & 1.75 & 0.033 & 2.14 & 0.099 & 1.57 & 0.056 & 1.60 & 0.255 \\
\hline \multirow[t]{2}{*}{2.65} & 41 & 1 & 3.68 & 0.002 & 2.13 & 0.101 & 1.61 & 0.048 & 4.05 & 0.017 \\
\hline & & 5 & 9.09 & $<0.001$ & 2.22 & 0.089 & 4.20 & 0.001 & 9.83 & 0.003 \\
\hline \multirow[t]{3}{*}{0.5603} & 44 & 0.5 & 1.26 & 0.138 & 1.43 & 0.254 & 1.13 & 0.471 & 3.32 & 0.014 \\
\hline & & 1 & 2.73 & 0.005 & 1.71 & 0.156 & 1.93 & 0.012 & 9.62 & 0.006 \\
\hline & & 5 & 6.76 & 0.001 & 2.40 & 0.141 & 3.04 & 0.004 & 55.46 & $>0.000$ \\
\hline
\end{tabular}

Gene expression values are fold changes relative to untreated control cells. Statistically significant relative expression changes are highlighted in bold. $p$-Values were calculated with the independent two-sample t-test. 

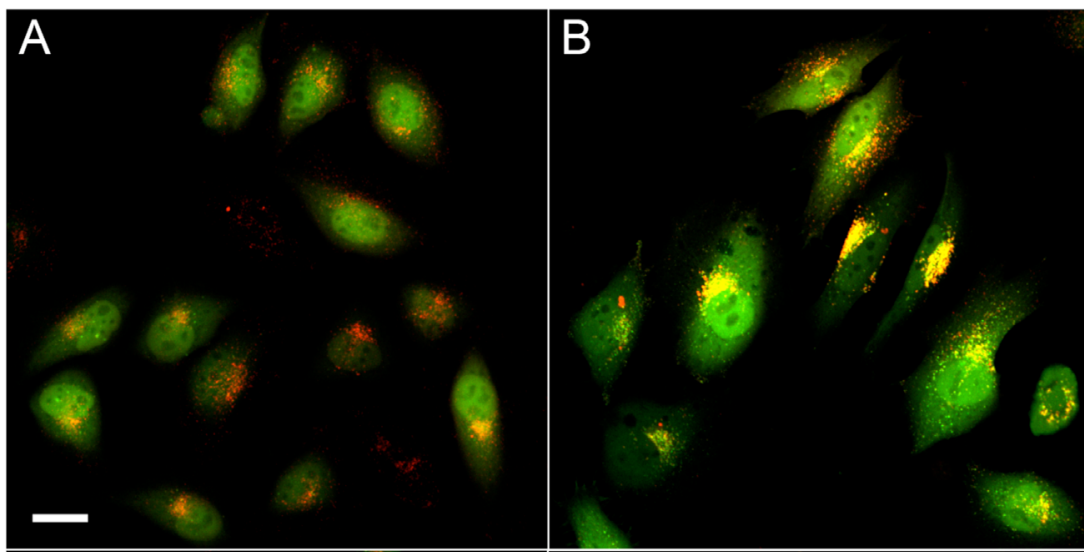

Figure 5. Representative confocal images of HeLa cells stably expressing GFP-mRFP-LC3B after $6 \mathrm{~h}$ treatment. Merged images of red and green fluorescent scans of untreated cells (A), cells treated with bafilomycin $(100 \mathrm{nM})(\mathrm{B})$, rapamycin $(500 \mathrm{nM})(\mathrm{C})$ or with $0.5 \mu \mathrm{M}$ analogue 44 (D). The scale bar on panel (A) represents $30 \mu \mathrm{m}$. (E) Quantification of images $A-D$, error bars represent standard deviation, ${ }^{* *} p<0.01$ (Student's t-test, control vs. treatment).
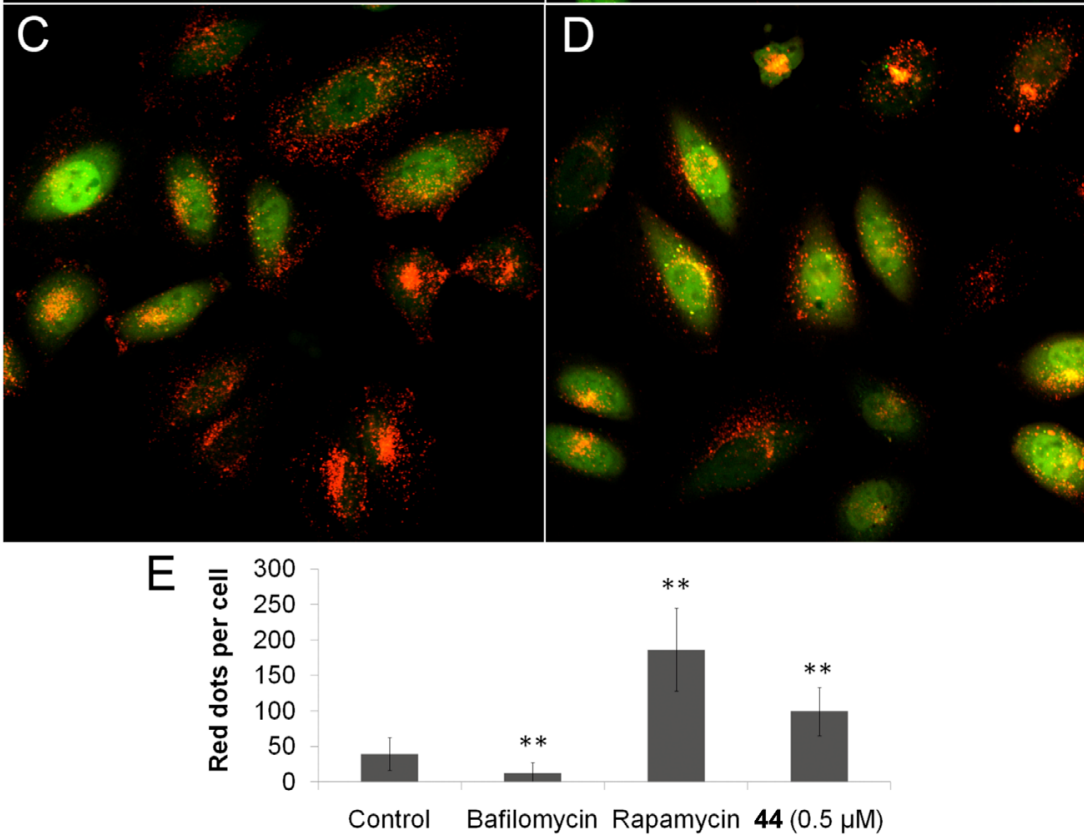

size relative to that in the control animals $(n=8)$ was detected after day 23 in the treated group $(n=9)$. The tumour mass remained significantly lower in the treated animals even weeks after the final administration (Fig. 8).

\section{Conclusion}

The present report has described the synthesis of novel Mannich-type curcumin derivatives as a new class of potential anticancer agents. Screening of these analogues for cytotoxicity, coupled with biochemical and gene expression studies, revealed that they have significantly better NF-кB-inhibitory activity than the parent compound curcumin. The favourable in vitro anticancer results led to in vivo tests with the lead compound 44. This compound effectively reduced the size of human lung tumours in SCID mice and displayed little toxicity.
Together with the noteworthy in vitro data, this suggests that $\mathbf{4 4}$ may potentially be an effective chemotherapeutic agent.

\section{Experimental}

\section{Chemistry}

General procedure for the synthesis of 7-52

To a stirred solution of the appropriate Mannich precursor 5 $(0.79 \mathrm{mmol})$ in $1.25 \mathrm{~mL}$ dry DMF under an argon atmosphere, $\mathrm{B}_{2} \mathrm{O}_{3}$ (55 mg, 1 equiv.) was added. The reaction mixture was stirred at $75^{\circ} \mathrm{C}$ for $30 \mathrm{~min}$, followed by the addition of $\mathrm{B}(\mathrm{OBu})_{3}$ ( $427 \mu \mathrm{L}, 2$ equiv.) and the appropriate aldehyde ( 2 equiv.). After stirring for $5 \mathrm{~min}$, morpholinium chloroacetate $(36 \mathrm{mg}$, $25 \mathrm{~mol} \%$ ) was added to the solution. The resulting mixture was stirred for a further $4 \mathrm{~h}$ at $75^{\circ} \mathrm{C}$ and monitored by TLC 


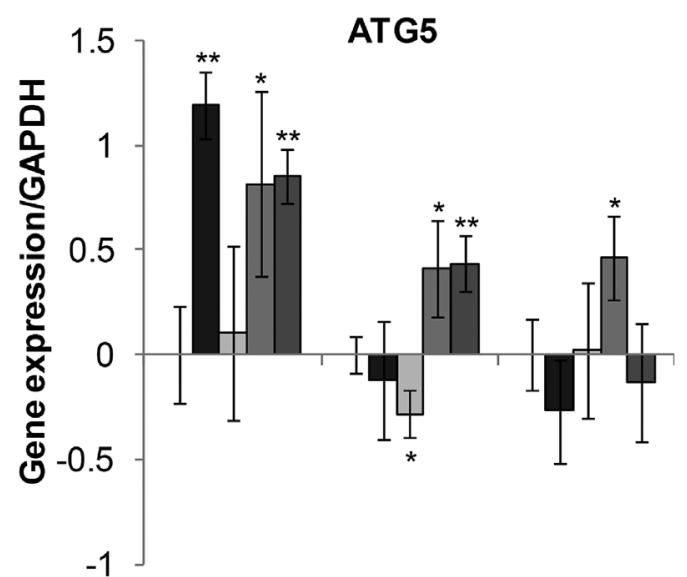

$6 \mathrm{~h}$

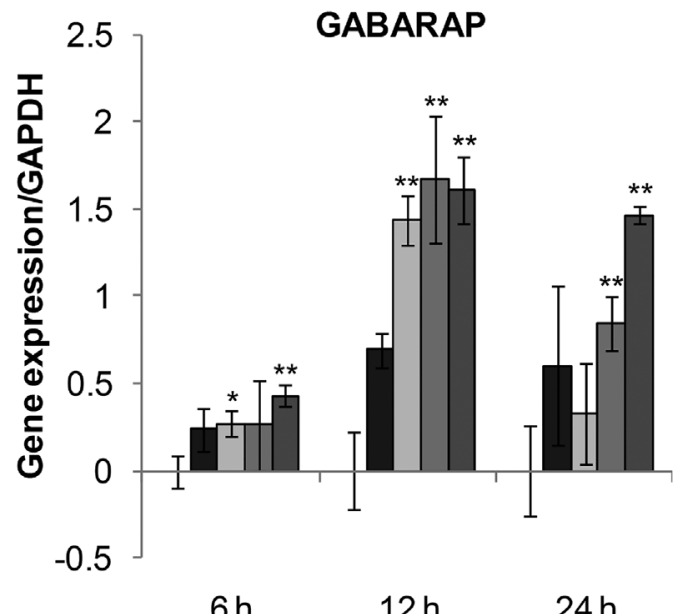

$6 \mathrm{~h}$

$12 \mathrm{~h}$

$24 \mathrm{~h}$

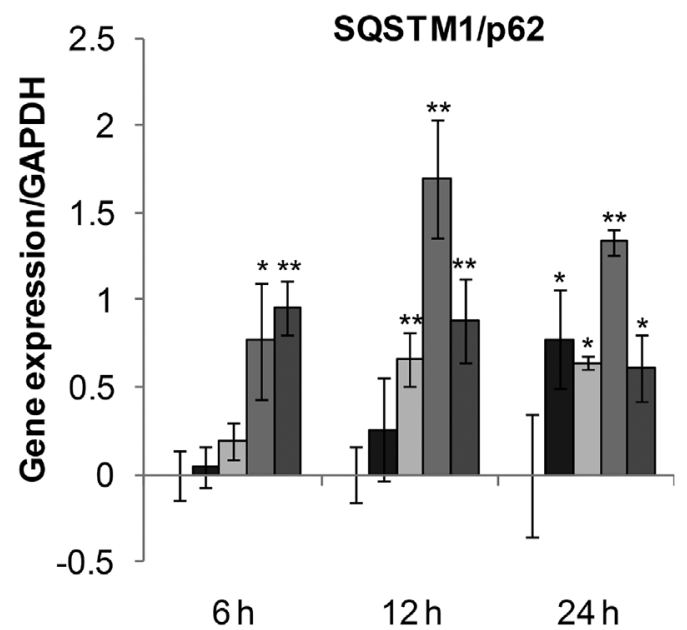

BECN1

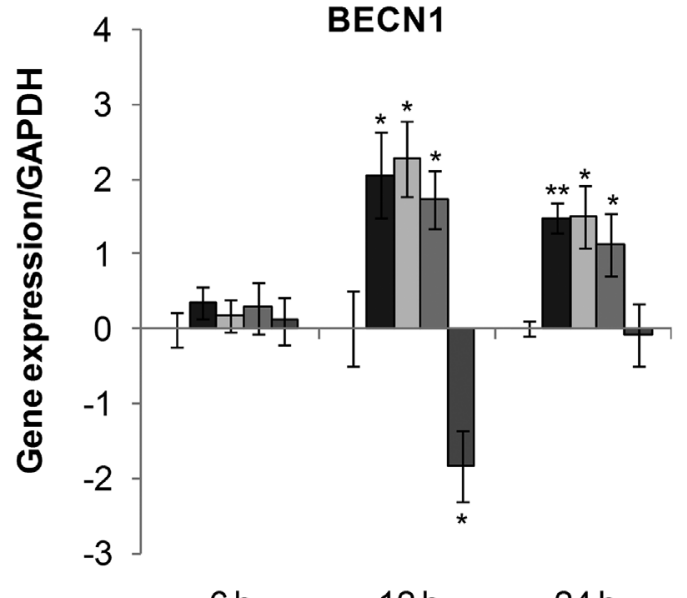

$12 \mathrm{~h} \quad 24 \mathrm{~h}$

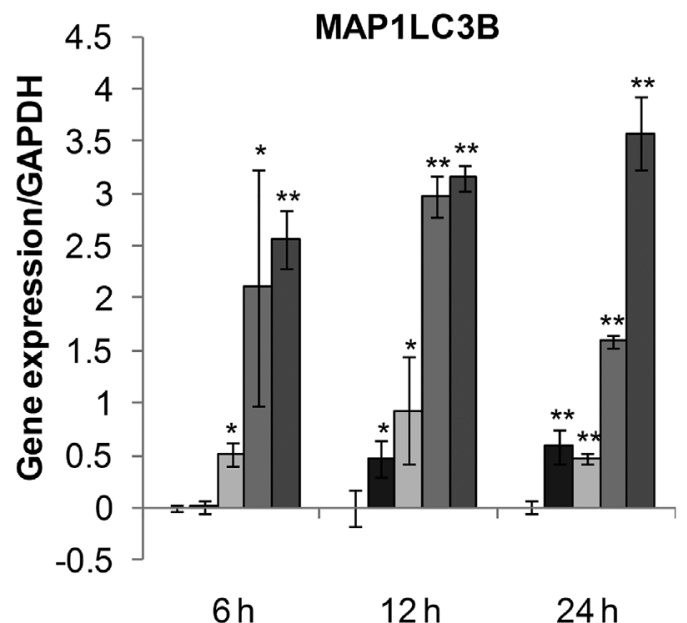

$\square$ Control

- Rapamycin

$\square 440.5 \mu \mathrm{M}$

$\square 441 \mu \mathrm{M}$

$\square 445 \mu \mathrm{M}$

Figure 6. QRT-PCR anylysis of autophagy related genes. A549 cells were treated with rapamycin ( $500 \mathrm{nM})$ or with $44(0.5,1$ or $5 \mu \mathrm{M})$. Expression is plotted on a $\log _{2}$ scale. Error bars represent standard deviation $(n=3),{ }^{*} p<0.05 ;{ }^{* *} p<0.01$ (Student's t-test, time matched control vs. treatment). 
A

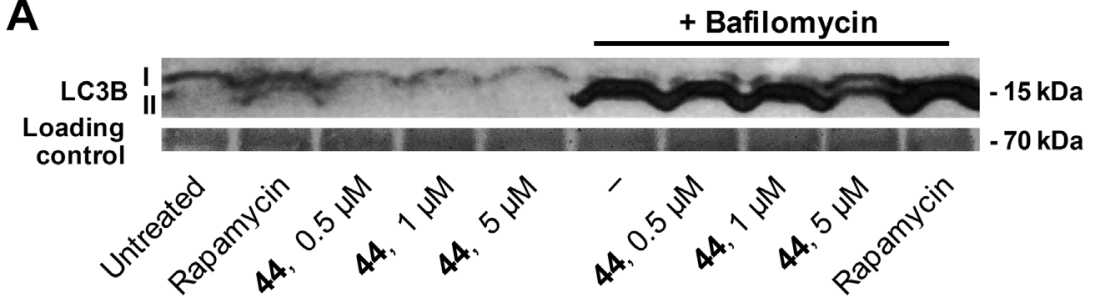

Figure 7. Western blot analysis of $L C 3 B$ and p62 protein expression. (A, B) LC3B expression. A549 cells were treated with rapamycin $(500 \mathrm{nM})$, bafilomycin $(100 \mathrm{nM})$, or with 44 $(0.5,1$ or $5 \mu \mathrm{M})$ and their combinations for $6 \mathrm{~h}$. (C, D) p62 expression. A549 cells were treated with rapamycin $(500 \mathrm{nM})$, or with $\mathbf{4 4}$ $(0.5 \mu \mathrm{M})$ for $24 \mathrm{~h}$.

B

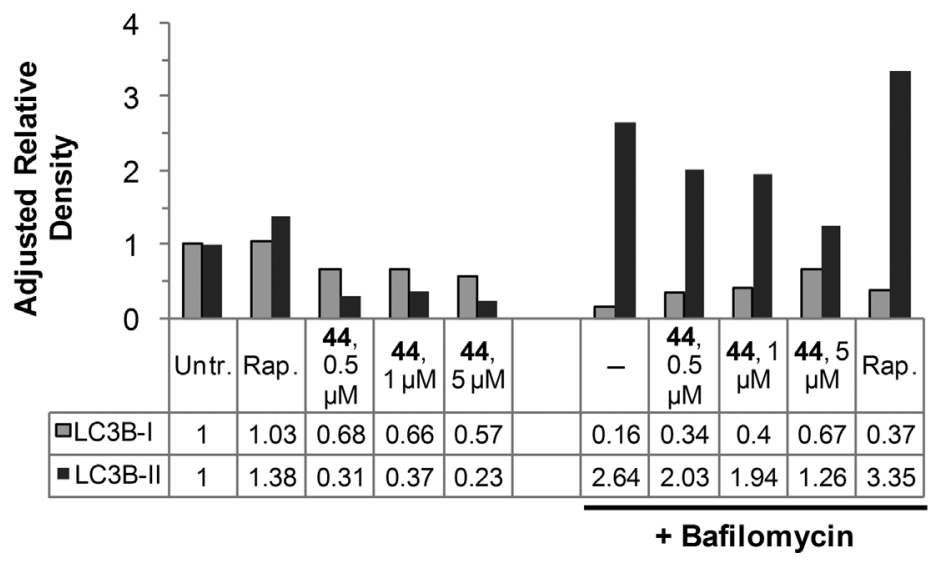

C

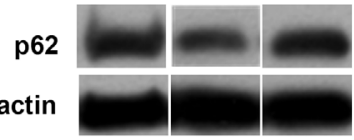

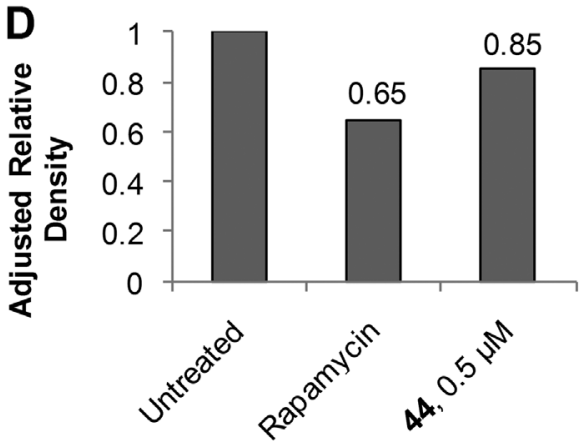

(eluent: $\mathrm{CHCl}_{3} / \mathrm{MeOH}$ mixture). After the mixture had been cooled to room temperature, $5 \mathrm{~mL}$ of $5 \mathrm{w} / \mathrm{w} \%$ aqueous $\mathrm{AcOH}$ solution was added dropwise in $15 \mathrm{~min}$ to quench the reaction, and the mixture was then stirred for a further $1 \mathrm{~h}$ at $75^{\circ} \mathrm{C}$. In the event of precipitation, the solid formed was collected by filtration, washed with water $(2 \times 25 \mathrm{~mL})$ and dried in vacuum. Crude products were recrystallised from either $\mathrm{Et}_{2} \mathrm{O}$ or $\mathrm{Et}_{2} \mathrm{O} / \mathrm{EtOH}$ mixtures. In the event of unsuccessful precipitation, the reaction mixture was extracted with EtOAc $(2 \times 20 \mathrm{~mL})$. The combined organic layers were washed with $40 \mathrm{~mL}$ of $5 \mathrm{w} / \mathrm{w} \%$ aqueous $\mathrm{NaHCO}_{3}$ solution and brine, dried over $\mathrm{Na}_{2} \mathrm{SO}_{4}$, evaporated and purified by column chromatography on silica (eluent: $\mathrm{CHCl}_{3} / \mathrm{MeOH}$ mixture).

The InChl codes of the investigated compounds together with some biological activity data are provided as Supporting Information.
Figure 8. In vivo efficacy study of 44 in the A549 xenograft model. Error bars represent SEM. ${ }^{*} p<0.05$, Student's t-test. 
Multigram-scale synthesis of lead compound $44-a$ representative example

Lead compound $\mathbf{4 4}$ was synthesised by the general procedure, starting from $5 \mathrm{~g}(19.3 \mathrm{mmol})$ of the corresponding Mannich precursor 5. A yellow solid precipitated during the acidic degradation of the boron complex. The solid formed was collected by vacuum filtration, and washed with water $(2 \times 25 \mathrm{~mL})$. After drying, the crude product was washed with $100 \mathrm{~mL} \mathrm{Et}{ }_{2} \mathrm{O} / \mathrm{EtOH}$ mixture (4:1) and left at room temperature for $3 \mathrm{~h}$. The solid was then filtered, washed with $\mathrm{Et}_{2} \mathrm{O}(50 \mathrm{~mL})$ and dried in vacuum, yielding $5.2 \mathrm{~g}(57 \%)$ of 44 as a paleyellow powder.

\section{N-((E)-5-(3,4-Dimethoxyphenyl)-2-((E)-3-(3,4-dimethoxy-} phenyl)acryloyl)-3-oxo-1-phenyl-pent-4-enyl)acetamide (7) $\mathrm{C}_{32} \mathrm{H}_{33} \mathrm{NO}_{7}, \mathrm{M}=543.2$; yield: $43 \%$; m.p.: $191-193^{\circ} \mathrm{C}$. ${ }^{1} \mathrm{H}$ NMR $\left(500.13 \mathrm{MHz}, \mathrm{DMSO}-d_{6}\right): \delta 1.70\left(s, 3 \mathrm{H}, \mathrm{CH}_{3} \mathrm{C}(\mathrm{O})\right), 3.78(s, 12 \mathrm{H}$, $\left.\mathrm{OCH}_{3}\right), 5.03-5.20(m, 1 \mathrm{H}, \mathrm{C}(\mathrm{O}) \mathrm{CHC}(\mathrm{O})), 5.66-5.85(m, 1 \mathrm{H}$, $\mathrm{NHCH}), 6.82-7.09(m, 4 \mathrm{H}),, 7.00-7.04(m, 10 \mathrm{H}), 7.66(d, 1 \mathrm{H}$, $J=15.0 \mathrm{~Hz}), 8.41(s, 1 \mathrm{H}, \mathrm{NHC}(\mathrm{O})) ;{ }^{13} \mathrm{C} \mathrm{NMR}(125.76 \mathrm{MHz}$, DMSO- $\left.d_{6}\right): \delta 23.6,52.7,56.5,68.1,111.49,111.53,112.5,112.6$, $124.0,124.3,124.4,124.7,127.6,127.9,128.0,128.4,129.0$, $142.3,144.85,144.92,149.9,152.3,152.4,169.0,192.8,193.2$; IR (FTIR, $\mathrm{cm}^{-1}$ ): 982, 1022, 1142, 1263, 1514, 1591, 1653, 2361 and 3298; MS (ES, neg. mode) $\mathrm{m} / \mathrm{z}=542.2[\mathrm{M}-\mathrm{H}]^{-}$.

N-((E)-1-(4-Chlorophenyl)-5-(3,4-dimethoxyphenyl)-2-((E)3-(3,4-dimethoxyphenyl)acryloyl)-3-oxopent-4-enyl)acetamide (8)

$\mathrm{C}_{32} \mathrm{H}_{32} \mathrm{CINO}_{7}, \mathrm{M}=577.2$, yield: $45 \%$; ${ }^{1} \mathrm{H}$ NMR $(500.13 \mathrm{MHz}$, DMSO- $\left.d_{6}\right)$ : $\delta 1.72\left(s, 3 \mathrm{H}, \mathrm{CH}_{3} \mathrm{C}(\mathrm{O})\right), 3.77-3.83\left(m, 12 \mathrm{H}, \mathrm{OCH}_{3}\right)$, $5.15(d, 1 \mathrm{H}, J=10.7 \mathrm{~Hz}, \mathrm{C}(\mathrm{O}) \mathrm{CHC}(\mathrm{O})), 5.74(t, 1 \mathrm{H}, J=9.6 \mathrm{~Hz}$, $\mathrm{NHCH}), 6.94-7.06(m, 4 \mathrm{H}), 7.21-7.46(m, 9 \mathrm{H}), 7.71(d, 1 \mathrm{H}$, $J=16.2 \mathrm{~Hz}), 8.46(d, 1 \mathrm{H}, J=8.8 \mathrm{~Hz}, \mathrm{NHC}(\mathrm{O})) ;{ }^{13} \mathrm{C} \mathrm{NMR}$ $\left(125.76 \mathrm{MHz}, \mathrm{DMSO}-d_{6}\right): \delta, 23.5,52.1,56.5,67.8,111.50$, $111.52,112.5,112.6,123.8,124.4,124.5,124.8,127.5,127.8$, $129.0,130.3,132.5,141.3,145.1,145.2,149.8,149.9,152.4$, 152.5, 169.2, 192.6, 192.9; IR (FTIR, $\mathrm{cm}^{-1}$ ): 984, 1022, 1142, 1263, 1512, 1594, 1653, 2362 and 3294; MS (ES, neg. mode) $m / z=576.2[\mathrm{M}-\mathrm{H}]^{-}$.

N-((E)-5-(3,4-Dimethoxyphenyl)-2-((E)-3-(3,4-dimethoxyphenyl)acryloyl)-1-(4-fluorophenyl)-3-oxopent-4-enyl)acetamide (9)

$\mathrm{C}_{32} \mathrm{H}_{32} \mathrm{FNO}_{7}, \mathrm{M}=561.2$; yield: $47 \%$; m.p.: $200-202^{\circ} \mathrm{C} ;{ }^{1} \mathrm{H}$ NMR $\left(500.13 \mathrm{MHz}, \mathrm{CDCl}_{3}\right): \delta 1.95\left(s, 3 \mathrm{H}, \mathrm{CH}_{3} \mathrm{C}(\mathrm{O})\right), 3.89(s, 12 \mathrm{H}$, $\left.\mathrm{OCH}_{3}\right), 4.75(d, 1 \mathrm{H}, J=6.9 \mathrm{~Hz}, \mathrm{C}(\mathrm{O}) \mathrm{CHC}(\mathrm{O})), 5.97(t, 1 \mathrm{H}$, $J=7.8 \mathrm{~Hz}, \mathrm{NHCH}), 6.70(d, 1 \mathrm{H}, J=13.1 \mathrm{~Hz}), 6.73(d, 1 \mathrm{H}$, $J=13.4 \mathrm{~Hz}), 6.80-6.86(m, 2 \mathrm{H}), 6.96(d, 2 \mathrm{H}, J=8.5 \mathrm{~Hz}), 7.00$ $7.04(m, 2 \mathrm{H}), 7.05-7.15(m, 3 \mathrm{H}), 7.34-7.40(m, 2 \mathrm{H}), 7.53(d, 1 \mathrm{H}$, $J=15.6 \mathrm{~Hz}), 7.60(d, 1 \mathrm{H}, J=15.6 \mathrm{~Hz}) ;{ }^{13} \mathrm{C}$ NMR $(125.76 \mathrm{MHz}$, $\left.\mathrm{CDCl}_{3}\right): \delta 22.9,51.4,55.5,66.8,109.6,109.7,110.6,114.9$, $115.1,121.2,121.7,123.5,123.7,126.4,128.1,128.2,135.5$, 145.0, 145.1, 148.9, 151.6, 160.6, 162.5, 169.0, 192.0, 194.5; IR (FTIR, $\mathrm{cm}^{-1}$ ): 984, 1022, 1142, 1261, 1514, 1591, 1651, 2361 and 3433; MS (ES, neg. mode) $\mathrm{m} / \mathrm{z}=560.2[\mathrm{M}-\mathrm{H}]^{-}$.
N-((E)-1-(3-Bromophenyl)-5-(3,4-dimethoxyphenyl)-2-((E)3-(3,4-dimethoxyphenyl)acryloyl)-3-oxopent-4-enyl)acetamide (10)

$\mathrm{C}_{32} \mathrm{H}_{32} \mathrm{BrNO}_{7}, \mathrm{M}=603.1$; yield: $48 \%$; m.p.: $215-218^{\circ} \mathrm{C} ;{ }^{1} \mathrm{H}$ NMR $\left(500.13 \mathrm{MHz}, \mathrm{CDCl}_{3}\right): \delta 1.98\left(s, 3 \mathrm{H}, \mathrm{CH}_{3} \mathrm{C}(\mathrm{O})\right), 3.91(s, 12 \mathrm{H}$, $\left.\mathrm{OCH}_{3}\right), 4.73(d, 1 \mathrm{H}, J=6.5 \mathrm{~Hz}, \mathrm{C}(\mathrm{O}) \mathrm{CHC}(\mathrm{O})), 5.94(t, 1 \mathrm{H}$, $J=8.0 \mathrm{~Hz}, \mathrm{NHCH}), 6.67(d, 1 \mathrm{H}, J=15.4 \mathrm{~Hz}), 6.74(d, 1 \mathrm{H}$, $J=15.7 \mathrm{~Hz}), 6.84(t, 2 \mathrm{H}, J=8.8 \mathrm{~Hz}), 7.02(s, 1 \mathrm{H}), 7.05(s, 1 \mathrm{H})$, 7.06-7.12 (m, 2H), 7.12-7.19 (m, 2H), 7.29-7.36 (m, 2H), 7.51 (d, $1 \mathrm{H}, J=15.5 \mathrm{~Hz}), 7.55(s, 1 \mathrm{H}) 7.62(d, 1 \mathrm{H}, J=15.9 \mathrm{~Hz}) ;{ }^{13} \mathrm{C} \mathrm{NMR}$ $\left(125.76 \mathrm{MHz}_{2} \mathrm{CDCl}_{3}\right)$ : $\delta 22.9,51.5,55.5,66.3,109.5,109.6$, $110.6,110.7,121.1,121.8,123.6,123.7,125.1,126.3,126.4$, $129.5,129.7,130.3,142.0,145.1,145.2,148.8,151.6,151.7$, 169.1, 191.8, 194.4; IR (FTIR, $\mathrm{cm}^{-1}$ ): 982, 1022, 1142, 1261, $1514,1591,1649$ and 3433; MS (ES, neg. mode) $\mathrm{m} / \mathrm{z}=620.1$ $[\mathrm{M}-\mathrm{H}]^{-}$.

N-((E)-5-(3,4-Dimethoxyphenyl)-2-((E)-3-(3,4-dimethoxyphenyl)acryloyl)-1-(2-fluorophenyl)-3-oxopent-4-enyl)acetamide (11)

$\mathrm{C}_{32} \mathrm{H}_{32} \mathrm{FNO}_{7}, \mathrm{M}=561.2$; yield: $41 \%$; m.p.: $209-212^{\circ} \mathrm{C} ;{ }^{1} \mathrm{H} N M R$ (500.13 MHz, CDCl 3$): \delta 1.98\left(s, 3 \mathrm{H}, \mathrm{CH}_{3} \mathrm{C}(\mathrm{O})\right), 3.89\left(\mathrm{~s}, 6 \mathrm{H}, \mathrm{OCH}_{3}\right)$, $3.92\left(s, 6 \mathrm{H}, \mathrm{OCH}_{3}\right), 4.92(d, 1 \mathrm{H}, J=6.5 \mathrm{~Hz}, \mathrm{C}(\mathrm{O}) \mathrm{CHC}(\mathrm{O})), 6.18(t$, $1 \mathrm{H}, J=7.6 \mathrm{~Hz}, \mathrm{NHCH}), 6.64(d, 1 \mathrm{H}, J=15.7 \mathrm{~Hz}), 6.75(d, 1 \mathrm{H}$, $J=15.8 \mathrm{~Hz}), 6.82(d, 1 \mathrm{H}, J=8.4 \mathrm{~Hz}), 6.87(d, 1 \mathrm{H} J=8.4 \mathrm{~Hz})$, 6.97-7.24 (m, 8H), 7.42-7.54 $(m, 2 \mathrm{H}), 7.68(d, 1 \mathrm{H}, J=15.8 \mathrm{~Hz})$; ${ }^{13} \mathrm{C}$ NMR $\left(125.76 \mathrm{MHz}_{1} \mathrm{CDCl}_{3}\right) \delta 22.9,47.9,55.5,64.5,109.5$, $109.6,110.5,110.6,115.0,115.1,121.6,121.8,123.6,123.7$, $123.9,126.4,128.9,129.0,129.2,144.6,145.2,148.8,148.9$, 151.5, 151.6, 169.0, 192.3, 194.4; IR (FTIR, cm $\left.{ }^{-1}\right)$ : 984, 1024, 1144, 1267, 1520, 1589, 1647, 1676 and 3296; MS (ES, neg. mode) $\mathrm{m} / \mathrm{z}=560.2[\mathrm{M}-\mathrm{H}]^{-}$.

N-((E)-1-(3,4-Difluorophenyl)-5-(3,4-dimethoxyphenyl)-2((E)-3-(3,4-dimethoxyphenyl)acryloyl)-3-oxopent-4-enyl)acetamide (12)

$\mathrm{C}_{32} \mathrm{H}_{31} \mathrm{~F}_{2} \mathrm{NO}_{7} ; \mathrm{M}=579.2$; yield: $51 \%$; m.p.: $218-220^{\circ} \mathrm{C} ;{ }^{1} \mathrm{H}$ NMR $\left(500.13 \mathrm{MHz}, \mathrm{CDCl}_{3}\right): \delta 1.97\left(\mathrm{~s}, 3 \mathrm{H}, \mathrm{CH}_{3} \mathrm{C}(\mathrm{O})\right), 4.71(\mathrm{~s}, 12 \mathrm{H}$, $\left.\mathrm{OCH}_{3}\right), 4.75(d, 1 \mathrm{H}, J=6.4 \mathrm{~Hz}, \mathrm{C}(\mathrm{O}) \mathrm{CHC}(\mathrm{O})), 5.91(t, 1 \mathrm{H}$, $J=7.8 \mathrm{~Hz}, \mathrm{NHCH}), 6.68(d, 1 \mathrm{H}, J=15.8 \mathrm{~Hz}), 6.74(d, 1 \mathrm{H}$, $J=15.8 \mathrm{~Hz}), 6.81-6.88(m, 2 \mathrm{H}), 7.03(d, 2 \mathrm{H}, J=9.1 \mathrm{~Hz})$, 7.05-7.17 $(m, 5 \mathrm{H}), 7.54(d, 1 \mathrm{H}, J=15.8 \mathrm{~Hz}), 7.62(d, 1 \mathrm{H}$, $J=15.8 \mathrm{~Hz}) ;{ }^{13} \mathrm{C}$ NMR $\left(125.76 \mathrm{MHz}_{,} \mathrm{CDCl}_{3}\right): \delta 22.8,51.3,55.5$, 66.4, 109.4, 109.7, 110.6, 110.7, 115.6, 115.8, 116.8, 116.9, $121.0,121.5,122.6,123.6,123.8,126.3,145.3,145.4,148.9$, 151.7, 151.8, 169.2, 191.7, 194.3; IR (FTIR, cm ${ }^{-1}$ ): 980, 1022, 1142, 1267, 1516, 1591, 1651 and 3292; MS (ES, neg, mode) $m / z=578.2[\mathrm{M}-\mathrm{H}]^{-}$.

N-((E)-1-(2,4-Dichlorophenyl)-5-(3,4-dimethoxyphenyl)-2((E)-3-(3,4-dimethoxyphenyl)acryloyl)-3-oxopent-4-enyl)acetamide (13)

$\mathrm{C}_{32} \mathrm{H}_{31} \mathrm{Cl}_{2} \mathrm{NO}_{7} ; \mathrm{M}=611.2$; yield: $55 \%$; m.p.: $224-226^{\circ} \mathrm{C}$; ${ }^{1} \mathrm{H}$ NMR $\left(500.13 \mathrm{MHz}, \mathrm{CDCl}_{3}\right): \delta 1.99\left(s, 3 \mathrm{H}, \mathrm{CH}_{3} \mathrm{C}(\mathrm{O})\right), 3.87$ $\left(s, 3 \mathrm{H}, \mathrm{OCH}_{3}\right), 3.89\left(s, 3 \mathrm{H}, \mathrm{OCH}_{3}\right), 3.92\left(s, 6 \mathrm{H}, \mathrm{OCH}_{3}\right), 4.99(d, 1 \mathrm{H}$, $J=4.7 \mathrm{~Hz}, \mathrm{C}(\mathrm{O}) \mathrm{CHC}(\mathrm{O})), 6.08(d d, 1 \mathrm{H}, J=8.3$ and $3.3 \mathrm{~Hz}$, 
$\mathrm{NHCH}), 6.51(d, 1 \mathrm{H}, J=15.7 \mathrm{~Hz}), 6.79-6.90(m, 3 \mathrm{H}), 6.97(s, 1 \mathrm{H})$, 7.03-7.11 $(m, 2 \mathrm{H}), 7.18(t, 2 \mathrm{H}, J=8.3 \mathrm{~Hz}), 7.36(s, 1 \mathrm{H}), 7.43(s$, $1 \mathrm{H}), 7.45(d, 1 \mathrm{H}, J=5.8 \mathrm{~Hz}), 7.52(d, 1 \mathrm{H}, J=8.3 \mathrm{~Hz}) ;{ }^{13} \mathrm{C} \mathrm{NMR}$ $\left(125.76 \mathrm{MHz} \mathrm{CDCl}_{3}\right): \delta 22.8,49.9,55.5,62.2,109.4,109.8$, $110.5,110.7,120.8,122.6,123.5,123.7,126.2,126.4,127.0$, $129.1,130.1,132.3,133.6,135.2,144.8,145.3,148.8,148.9$, 151.7, 169.1, 192.0, 194.8; IR (FTIR, $\mathrm{cm}^{-1}$ ): 982, 1022, 1140, 1265, 1514, 1591, 1649, 1672, 2359 and 3421; MS (ES, neg. mode) $\mathrm{m} / \mathrm{z}=610.2[\mathrm{M}-\mathrm{H}]^{-}$.

$N-((E)-5-(3,4-D i m e t h o x y p h e n y l)-2-((E)-3-(3,4-$ dimethoxyphenyl)acryloyl)-3-oxo-1-(4-(trifluoromethyl)phenyl)pent-4-enyl)acetamide (14)

$\mathrm{C}_{33} \mathrm{H}_{32} \mathrm{~F}_{3} \mathrm{NO}_{7} ; \mathrm{M}=611.2$; yield: $60 \%$; m.p.: $195-196^{\circ} \mathrm{C} ;{ }^{1} \mathrm{H}$ NMR (500.13 MHz, CDCl $)$ : $\delta 1.98\left(s, 3 \mathrm{H}, \mathrm{CH}_{3} \mathrm{C}(\mathrm{O})\right), 3.89\left(\mathrm{~s}, 3 \mathrm{H}, \mathrm{OCH}_{3}\right)$, $3.90\left(s, 6 \mathrm{H}, \mathrm{OCH}_{3}\right), 3.91\left(s, 3 \mathrm{H}, \mathrm{OCH}_{3}\right), 4.77(d, 1 \mathrm{H}, J=6.3 \mathrm{~Hz}$, $\mathrm{C}(\mathrm{O}) \mathrm{CHC}(\mathrm{O})), 6.01(d d$, overlapped peaks, $1 \mathrm{H}, J=7.4 \mathrm{~Hz}$, $\mathrm{NHCH}), 6.68(d, 1 \mathrm{H}, J=15.7 \mathrm{~Hz}), 6.75(d, 1 \mathrm{H}, J=15.4 \mathrm{~Hz})$, $6.84(t, 2 \mathrm{H}, J=8.4 \mathrm{~Hz}), 7.01(\mathrm{~s}, 1 \mathrm{H}), 7.04(s, 1 \mathrm{H}), 7.07-7.11(\mathrm{~m}$, $1 \mathrm{H}), 7.14(d, 2 \mathrm{H}, J=8.4 \mathrm{~Hz}), 7.49-7.58(m, 4 \mathrm{H}), 7.63(d, 1 \mathrm{H}$, $J=15.7 \mathrm{~Hz}) ;{ }^{13} \mathrm{C}$ NMR $\left(125.76 \mathrm{MHz} \mathrm{CDCl}_{3}\right): \delta 22.8,51.7,55.5$, $66.3,109.6,109.7,110.6,110.7,121.0,121.5,123.6,123.8$, $125.1,125.2,126.2,126.3,126.9,143.7,145.3,145.4,148.9$, 151.7, 151.8, 169.2, 191.8, 194.3; IR (FTIR, $\mathrm{cm}^{-1}$ ): 982, 1022, 1070, 1122, 1261, 1327, 1514, 1593, 1647, 1678 and 3294; MS (ES, neg. mode) $m / z=610.2[\mathrm{M}-\mathrm{H}]^{-}$.

N-((E)-1-(4-Bromothiophen-2-yl)-5-(3,4-dimethoxyphenyl)-2-((E)-3-(3,4-dimethoxyphenyl)acryloyl)-3oxopent-4-enyl)acetamide (15)

$\mathrm{C}_{30} \mathrm{H}_{30} \mathrm{BrNO}_{7} \mathrm{~S} ; \mathrm{M}=627.1$; yield: $35 \%$; m.p.: $188-190^{\circ} \mathrm{C}$; ${ }^{1} \mathrm{H}$ NMR $\left(500.13 \mathrm{MHz}\right.$, DMSO- $\left.d_{6}\right): \delta 1.72\left(s, 3 \mathrm{H}, \mathrm{CH}_{3} \mathrm{C}(\mathrm{O})\right)$, $3.76\left(s, 3 \mathrm{H}, \mathrm{OCH}_{3}\right), 3.77\left(\mathrm{~s}, 3 \mathrm{H}, \mathrm{OCH}_{3}\right), 3.79\left(\mathrm{~s}, 3 \mathrm{H}, \mathrm{OCH}_{3}\right), 3.80(\mathrm{~s}$, $\left.3 \mathrm{H}, \mathrm{OCH}_{3}\right), 5.24(d, 1 \mathrm{H}, J=10.8 \mathrm{~Hz}, \mathrm{C}(\mathrm{O}) \mathrm{CHC}(\mathrm{O})), 5.90(t, 1 \mathrm{H}$, $J=9.9 \mathrm{~Hz}, \mathrm{NHCH}), 6.95-7.04(m, 4 \mathrm{H}), 7.06(d, 1 \mathrm{H}, J=15.8 \mathrm{~Hz})$, $7.25(d, 1 \mathrm{H}, J=8.2 \mathrm{~Hz}), 7.28-7.34(m, 2 \mathrm{H}), 7.35(s, 1 \mathrm{H}), 7.46(s$, $1 \mathrm{H}), 7.54(d, 1 \mathrm{H}, J=15.8 \mathrm{~Hz}), 7.72(d, 1 \mathrm{H}, J=15.8 \mathrm{~Hz}), 8.51(d$, $\left.1 \mathrm{H}, J=8.7 \mathrm{~Hz}, \mathrm{C}(\mathrm{O}) \mathrm{NH}_{\imath}\right) ;{ }^{13} \mathrm{C} \mathrm{NMR}\left(125.76 \mathrm{MHz} \mathrm{CDCl}_{3}\right): \delta 22.9$, 51.7, 55.5, 55.6, 66.3, 109.6, 109.7, 110.6, 110.7, 121.0, 121.5, $123.7,123.9,125.2,126.2,126.3,126.9,143.7,145.3,145.4$, 148.9, 151.7, 151.8, 169.3, 191.8, 194.3; IR (FTIR, $\mathrm{cm}^{-1}$ ): 982, 1022, 1140, 1261, 1514, 1589, 1653 and 3427; MS (ES, neg. mode) $m / z=626.1[\mathrm{M}-\mathrm{H}]^{-}$.

$N-((E)-5-(3,4-D i m e t h o x y p h e n y l)-2-((E)-3-(3,4-d i m e t h o x y-$ phenyl)acryloyl)-3-oxo-1-(3-(trifluoromethyl)-phenyl)pent-4-enyl)acetamide (16)

$\mathrm{C}_{33} \mathrm{H}_{32} \mathrm{~F}_{3} \mathrm{NO}_{7} ; \mathrm{M}=611.2$; yield: $58 \%$; m.p.: $209-212^{\circ} \mathrm{C} ;{ }^{1} \mathrm{H} \mathrm{NMR}$ $\left(500.13 \mathrm{MHz}, \mathrm{CDCl}_{3}\right): \delta 1.98\left(s, 3 \mathrm{H}, \mathrm{CH}_{3} \mathrm{C}(\mathrm{O})\right), 3.89(s, 12 \mathrm{H}$, $\left.\mathrm{OCH}_{3}\right), 4.77(d, 1 \mathrm{H}, J=6.5 \mathrm{~Hz}, \mathrm{C}(\mathrm{O}) \mathrm{CHC}(\mathrm{O})), 6.02(t, 1 \mathrm{H}$, $J=7.4 \mathrm{~Hz}, \mathrm{NHCH}), 6.68(d, 1 \mathrm{H}, J=16.0 \mathrm{~Hz}), 6.75(d, 1 \mathrm{H}$, $J=16.0 \mathrm{~Hz}), 6.83(t, 2 \mathrm{H}, J=6.8 \mathrm{~Hz}), 7.00(s, 1 \mathrm{H}), 7.03(s, 1 \mathrm{H})$, 7.06-7.15 (m, 2H), 7.19 (s, 1H), 7.37-7.44 (m, 2H), 7.44-7.51 (m, $2 \mathrm{H}), 7.59(d, 1 \mathrm{H}, J=8.6 \mathrm{~Hz}), 7.67(s, 1 \mathrm{H}) ;{ }^{13} \mathrm{C} \mathrm{NMR}(125.76 \mathrm{MHz}$, $\left.\mathrm{CDCl}_{3}\right): \delta 22.8,51.7,55.5,66.3,109.5,109.6,110.6,121.0$, $121.6,122.7,123.2,123.6,123.7,124.0,126.3,128.6,130.8$,
145.2, 145.3, 148.8, 151.6, 169.3, 191.8, 194.3; IR (FTIR, $\left.\mathrm{cm}^{-1}\right)$ : $982,1024,1119,1163,1263,1333,1514,1593,1651,1670$ and 3294; MS (ES, neg. mode) $\mathrm{m} / \mathrm{z}=610.2[\mathrm{M}-\mathrm{H}]^{-}$.

N-((E)-1-(4-Butylphenyl)-5-(3,4-dimethoxyphenyl)-2-((E)-3(3,4-dimethoxyphenyl)acryloyl)-3-oxopent-4-en-1-yl)acetamide (17)

$\mathrm{C}_{36} \mathrm{H}_{41} \mathrm{NO}_{7} ; \mathrm{M}=599.3$; yield: $30 \%$; m.p.: $182-183^{\circ} \mathrm{C} ;{ }^{1} \mathrm{H}$ NMR $\left(500.13 \mathrm{MHz}, \mathrm{DMSO}-d_{6}\right): \delta 0.80\left(t, 3 \mathrm{H}, J=7.3 \mathrm{~Hz}, \mathrm{CH}_{3} \mathrm{CH}_{2}\right)$, 1.16-1.24 (m, 2H, $\left.\mathrm{CH}_{2} \mathrm{CH}_{2}\right), 1.38-1.47\left(\mathrm{~m}, 2 \mathrm{H}, \mathrm{CH}_{2} \mathrm{CH}_{2}\right), 1.69(\mathrm{~s}$, $\left.3 \mathrm{H}, \mathrm{CH}_{3} \mathrm{C}(\mathrm{O})\right), 2.45\left(t, 2 \mathrm{H}, J=7.5 \mathrm{~Hz}, \mathrm{CH}_{3} \mathrm{CH}_{2} \mathrm{Ph}\right), 3.76(s, 3 \mathrm{H}$, $\left.\mathrm{OCH}_{3}\right), 3.77\left(\mathrm{~s}, 3 \mathrm{H}, \mathrm{OCH}_{3}\right), 3.79\left(\mathrm{~s}, 3 \mathrm{H}, \mathrm{OCH}_{3}\right), 3.80\left(\mathrm{~s}, 3 \mathrm{H}, \mathrm{OCH}_{3}\right)$, $5.08(d, 1 \mathrm{H}, J=10.7 \mathrm{~Hz}, \mathrm{C}(\mathrm{O}) \mathrm{CHC}(\mathrm{O})), 5.72(t, 1 \mathrm{H}, J=9.9 \mathrm{~Hz}$, $\mathrm{NHCH}), 6.89(d, 1 \mathrm{H}, J=15.9 \mathrm{~Hz}), 6.95(d, 1 \mathrm{H}, J=8.2 \mathrm{~Hz})$, 6.97-7.03 $(m, 2 \mathrm{H}), 7.06(d, 2 \mathrm{H}, J=7.9 \mathrm{~Hz}), 7.18(d, 1 \mathrm{H}$, $J=8.4 \mathrm{~Hz}), 7.21-7.32(m, 4 \mathrm{H}), 7.33(s, 1 \mathrm{H}), 7.37(d, 1 \mathrm{H}$, $J=15.9 \mathrm{~Hz}), 7.65(d, 1 \mathrm{H}, J=15.9 \mathrm{~Hz}), 8.37(d, 1 \mathrm{H}, J=8.9 \mathrm{~Hz}$, $\mathrm{C}(\mathrm{O}) \mathrm{NH}) ;{ }^{13} \mathrm{C}$ NMR $\left(125.76 \mathrm{MHz}\right.$, DMSO- $\left.d_{6}\right): \delta 14.6,22.6,23.6$, $33.9,35.3,52.4,56.5,68.1,111.47,111.52,112.4,112.6,124.1$, $124.3,124.4,124.7,127.6,127.9,128.2,128.9,139.5,142.0$, 144.8, 149.8, 149.9, 152.3, 152.4, 169.0, 193.0, 193.3; IR (FTIR, $\mathrm{cm}^{-1}$ ): 983, 1022, 1140, 1265, 1514, 1591, 2359, 2933 and 3304; MS (ES, neg. mode) $\mathrm{m} / \mathrm{z}=598.2[\mathrm{M}-\mathrm{H}]^{-}$.

4-((E)-1-Acetamido-5-(3,4-dimethoxyphenyl)-2-((E)-3-(3,4dimethoxyphenyl)acryloyl)-3-oxopent-4-en-1-yl)benzoic acid (18)

$\mathrm{C}_{33} \mathrm{H}_{33} \mathrm{NO}_{9} ; \mathrm{M}=587.2$; yield: $62 \% ;{ }^{1} \mathrm{H} \quad \mathrm{NMR} \quad(500.13 \mathrm{MHz}$, DMSO- $\left.d_{6}\right): \delta 1.72\left(s, 3 \mathrm{H}, \mathrm{CH}_{3} \mathrm{C}(\mathrm{O})\right), 3.76\left(s, 3 \mathrm{H}, \mathrm{OCH}_{3}\right), 3.77$ $\left(s, 3 \mathrm{H}, \mathrm{OCH}_{3}\right), 3.80\left(s, 3 \mathrm{H}, \mathrm{OCH}_{3}\right), 3.81\left(s, 3 \mathrm{H}, \mathrm{OCH}_{3}\right), 5.19(d, 1 \mathrm{H}$, $J=10.5 \mathrm{~Hz}, \mathrm{C}(\mathrm{O}) \mathrm{CHC}(\mathrm{O})), 5.80(t, 1 \mathrm{H}, J=10.2 \mathrm{~Hz}, \mathrm{NHCH})$, 6.90-6.99 $(m, 3 \mathrm{H}), 7.00-7.06(m, 2 \mathrm{H}), 7.21(d, 1 \mathrm{H}, J=8.4 \mathrm{~Hz})$, $7.24(s, 1 \mathrm{H}), 7.32(d, 1 \mathrm{H}, J=8.4 \mathrm{~Hz}), 7.35(s, 1 \mathrm{H}), 7.42(d, 1 \mathrm{H}$, $J=15.9 \mathrm{~Hz}), 7.50(d, 2 \mathrm{H}, J=8.2 \mathrm{~Hz}), 7.70(d, 1 \mathrm{H}, J=15.9 \mathrm{~Hz})$, $7.85(d, 2 \mathrm{H}, J=8.2 \mathrm{~Hz}), 8.49(d, 1 \mathrm{H}, J=8.9 \mathrm{~Hz}, \mathrm{C}(\mathrm{O}) \mathrm{NH})$; ${ }^{13} \mathrm{C}$ NMR $\left(125.76 \mathrm{MHz}\right.$, DMSO- $\left.d_{6}\right): \delta 22.6,51.6,55.5,66.7$, $110.61,110.64,111.5,111.6,122.9,123.5,123.6,123.8,126.6$, $126.9,127.7,129.1,144.1,144.2,146.2,148.9,149.0,151.4$, 151.5, 167.0, 168.3, 191.7, 192.0. IR (FTIR, cm ${ }^{-1}$ ): 980, 1022, 1140, 1163, 1267, 1515, 1591, 1652, 2361 and 3414; MS (ES, neg. mode) $m / z=586.2[\mathrm{M}-\mathrm{H}]^{-}$.

4-((E)-1-Acetamido-5-(3,4-dimethoxyphenyl)-2-((E)-3-(3,4dimethoxy-phenyl)acryloyl)-3-oxopent-4-en-1-yl)-2methoxyphenyl acetate (19)

$\mathrm{C}_{35} \mathrm{H}_{37} \mathrm{NO}_{10}$; $\mathrm{M}=631.2$; yield: $49 \%$; m.p.: $180-182^{\circ} \mathrm{C} ;{ }^{1} \mathrm{H}$ NMR $\left(500.13 \mathrm{MHz}, \mathrm{CDCl}_{3}\right): \delta 1.94\left(s, 3 \mathrm{H}, \mathrm{CH}_{3} \mathrm{C}(\mathrm{O})\right), 2.26(\mathrm{~s}, 3 \mathrm{H}$, $\left.\mathrm{CH}_{3} \mathrm{C}(\mathrm{O}) \mathrm{O}-\mathrm{Ar}\right), 3.79\left(\mathrm{~s}, 3 \mathrm{H}, \mathrm{OCH}_{3}\right), 3.89$ (bs, overlapped peaks, $\left.12 \mathrm{H}, \mathrm{OCH}_{3}\right), 4.76(d, 1 \mathrm{H}, J=7.0 \mathrm{~Hz}, \mathrm{C}(\mathrm{O}) \mathrm{CHC}(\mathrm{O})), 5.99(t, 1 \mathrm{H}$, $J=7.7 \mathrm{~Hz}, \mathrm{NHCH}), 6.72(d, 1 \mathrm{H}, J=10.5 \mathrm{~Hz}), 6.75(d, 1 \mathrm{H}$, $J=10.5 \mathrm{~Hz}), 6.80-6.86(m, 2 \mathrm{H}), 6.93$ (bs, 2H), 7.04 (bs, overlapped peaks, 4H), 7.08-7.15 $(m, 2 \mathrm{H}), 7.56(d, 1 \mathrm{H}$, $J=15.7 \mathrm{~Hz}), 7.60(d, 1 \mathrm{H}, J=15.7 \mathrm{~Hz}) ;{ }^{13} \mathrm{C} \mathrm{NMR}(125.76 \mathrm{MHz}$, $\left.\mathrm{CDCl}_{3}\right): \delta 20.2,22.9,51.8,55.5,66.9,109.6,110.6,111.5,118.3$, $121.4,121.6,122.4,123.5,123.7,126.4,138.7,144.9,145.0$, 148.8, 151.5, 168.4, 169.0, 192.0, 194.5; IR (FTIR, $\left.\mathrm{cm}^{-1}\right)$ : 547, 
$767,802,979,1024,1143,1222,1514,1593,1647$ and 2166; MS (ES, neg. mode) $\mathrm{m} / \mathrm{z}=630.2[\mathrm{M}-\mathrm{H}]^{-}$.

4,4'-((1E,6E)-4-(Acetamido(phenyl)methyl)-3,5dioxohepta-1,6-diene-1,7-diyl)bis(2-methoxy-4,1phenylene)diacetate (20)

$\mathrm{C}_{34} \mathrm{H}_{33} \mathrm{NO}_{9}, \mathrm{M}=599.2$; yield: $45 \%$; ${ }^{1} \mathrm{H}$ NMR $(500.13 \mathrm{MHz}$, DMSO- $\left.d_{6}\right): \delta 1.98\left(s, 3 \mathrm{H}, \mathrm{CH}_{3} \mathrm{C}(\mathrm{O})\right), 2.24(\mathrm{~s}, 3 \mathrm{H}, \mathrm{OAc}), 2.26(\mathrm{~s}$, $3 \mathrm{H}, \mathrm{OAc}), 3.78\left(\mathrm{~s}, 3 \mathrm{H}, \mathrm{OCH}_{3}\right), 3.82\left(s, 3 \mathrm{H}, \mathrm{OCH}_{3}\right), 5.21(d, 1 \mathrm{H}$, $J=10.7 \mathrm{~Hz}, \mathrm{C}(\mathrm{O}) \mathrm{CHC}(\mathrm{O})), 5.80(t, 1 \mathrm{H}, J=9.8 \mathrm{~Hz}, \mathrm{CHNH}), 7.03-$ $7.52(\mathrm{~m}, 14 \mathrm{H}), 7.73(d, 1 \mathrm{H}, J=15.7 \mathrm{~Hz}), 8.46(d, 1 \mathrm{H}, J=9.1 \mathrm{~Hz})$; ${ }^{13} \mathrm{C}$ NMR $\left(125.76 \mathrm{MHz}\right.$, DMSO- $\left.d_{6}\right): \delta 21.2,23.6,52.7,56.9,68.0$, $113.3,122.6,122.9,124.2,124.3,126.4,126.7,128.1,128.4$, 129.1, 133.8, 134.1, 142.0, 142.2, 142.3, 144.0, 144.1, 152.0, 152.1, 169.1, 169.2, 169.2, 193.1, 193.5; IR (FTIR, cm $\left.{ }^{-1}\right): 594$, 702, 985, 1034, 1126, 1200, 1512, 1612, 1767, 2355 and 3319 MS (ES, neg. mode) $\mathrm{m} / \mathrm{z}=598.2[\mathrm{M}-\mathrm{H}]^{-}$.

N-((E)-1-(3-Bromophenyl)-5-(4-hydroxy-3-

methoxyphenyl)-2-((E)-3-(4-hydroxy-3-methoxyphenyl)acryloyl)-3-oxopent-4-enyl)acetamide (21)

$\mathrm{C}_{30} \mathrm{H}_{28} \mathrm{BrNO}_{7} ; \mathrm{M}=593.1$; yield: $45 \%$; m.p.: $146-148^{\circ} \mathrm{C} ;{ }^{1} \mathrm{H} \mathrm{NMR}$ $\left(500.13 \mathrm{MHz}, \mathrm{CDCl}_{3}\right): \delta 1.98\left(\mathrm{~s}, 3 \mathrm{H}, \mathrm{CH}_{3} \mathrm{C}(\mathrm{O})\right), 3.90$ (bs, 6H, $\left.\mathrm{OCH}_{3}\right), 4.72(d, 1 \mathrm{H}, J=6.3 \mathrm{~Hz}, \mathrm{C}(\mathrm{O}) \mathrm{CHC}(\mathrm{O})), 5.94(t, 1 \mathrm{H}$, $J=7.6 \mathrm{~Hz}, \mathrm{CHNH}), 6.17(\mathrm{~s}$, overlapped peaks, $2 \mathrm{H}, 2 \mathrm{xOH})$, $6.65(d, 1 \mathrm{H}, J=15.9 \mathrm{~Hz}), 6.72(d, 1 \mathrm{H}, J=15.9 \mathrm{~Hz}), 6.88(t, 3 \mathrm{H}$, $J=8.4 \mathrm{~Hz}), 6.97-7.06(m, 3 \mathrm{H}), 7.06-7.18(m, 2 \mathrm{H}), 7.31(d, 1 \mathrm{H}$, $J=7.9 \mathrm{~Hz}), 7.50(d, 1 \mathrm{H}, J=15.9 \mathrm{~Hz}), 7.55(s, 1 \mathrm{H}), 7.60(d, 1 \mathrm{H}$, $J=15.9 \mathrm{~Hz}) ;{ }^{13} \mathrm{C}$ NMR $\left(125.76 \mathrm{MHz} \mathrm{CDCl}_{3}\right): \delta 22.9,51.5,55.6$, $66.3,109.3,109.5,114.4,114.5,120.8,121.4,122.3,123.9$, 124.2, 125.1, 125.9, 129.5, 129.7, 130.3, 142.0, 145.3, 145.4, 146.4, 148.6, 148.7, 169.3, 191.8, 194.4; IR (FTIR, cm ${ }^{-1}$ ): 980 , 1032, 1124, 1163, 1273, 1429, 1514, 1578, 1653 and 3394; MS (ES, neg. mode) $\mathrm{m} / \mathrm{z}=592.1[\mathrm{M}-\mathrm{H}]^{-}$.

$N-((E)-1-(4-C h l o r o p h e n y l)-5-(4-h y d r o x y-3-m e t h o x y-$ phenyl)-2-((E)-3-(4-hydroxy-3-methoxyphenyl)acryloyl)-3oxopent-4-en-1-yl)acetamide (22)

$\mathrm{C}_{30} \mathrm{H}_{28} \mathrm{ClNO}_{7}, \mathrm{M}=549.2$; yield: $47 \%$. ${ }^{1} \mathrm{H}$ NMR $(500.13 \mathrm{MHz}$, DMSO- $\left.d_{6}\right): \delta 1.70\left(s, 3 \mathrm{H}, \mathrm{CH}_{3} \mathrm{C}(\mathrm{O})\right), 3.77\left(\mathrm{~s}, 3 \mathrm{H}, \mathrm{OCH}_{3}\right), 3.81(\mathrm{~s}$, $\left.3 \mathrm{H}, \mathrm{OCH}_{3}\right), 5.07(d, 1 \mathrm{H}, J=10.6 \mathrm{~Hz}, \mathrm{C}(\mathrm{O}) \mathrm{CHC}(\mathrm{O})), 5.71(t, 1 \mathrm{H}$, $J=9.9 \mathrm{~Hz}, \mathrm{NHCH}), 6.76(d, 1 \mathrm{H}, J=8.2 \mathrm{~Hz}), 6.81(d, 1 \mathrm{H}$, $J=8.0 \mathrm{~Hz}), 6.86(d, 1 \mathrm{H}, J=15.7 \mathrm{~Hz}), 6.94(d, 1 \mathrm{H}, J=15.7 \mathrm{~Hz})$, $7.09(d, 1 \mathrm{H}, J=8.0 \mathrm{~Hz}), 7.19(d, 1 \mathrm{H}, J=8.0 \mathrm{~Hz}), 7.22(b s, 1 \mathrm{H})$, $7.29-7.43(m, 6 \mathrm{H}), 7.64(d, 1 \mathrm{H}, J=15.7 \mathrm{~Hz}), 8.42(d, 1 \mathrm{H}, \mathrm{C}(\mathrm{O}) \mathrm{NH}$, $J=8.9 \mathrm{~Hz}), 9.73(2 \mathrm{xs}$, overlapped peaks, $2 \mathrm{H}, 2 \mathrm{xOH}) ;{ }^{13} \mathrm{C} \mathrm{NMR}$ $\left(125.76 \mathrm{MHz}\right.$, DMSO- $\left.d_{6}\right)$ : $\delta 21.7,51.3,55.7,67.0,111.6,115.6$, $115.7,122.0,122.5,123.7,124.1,125.4,125.7,128.1,129.4$, $131.6,140.5,144.6,144.7,147.9,148.0,150.0,150.1,168.3$, 191.7, 192.0; MS (ES, neg. mode) $\mathrm{m} / \mathrm{z}=548.1[\mathrm{M}-\mathrm{H}]^{-}$.

$N-((E)-5-(3-H y d r o x y-4-m e t h o x y p h e n y l)-2-((E)-3-(3-$ hydroxy-4-methoxyphenyl)acryloyl)-3-oxo-1-phenyl-pent4-en-1-yl)acetamide (23)

$\mathrm{C}_{30} \mathrm{H}_{29} \mathrm{NO}_{7} ; \mathrm{M}=515.2$; yield: $45 \%$; m.p.: $182-185^{\circ} \mathrm{C} ;{ }^{1} \mathrm{H}$ NMR $\left(500.13 \mathrm{MHz}, \mathrm{DMSO}-d_{6}\right)$ : $\delta 1.65\left(s, 3 \mathrm{H}, \mathrm{CH}_{3} \mathrm{C}(\mathrm{O})\right), 3.78(s, 3 \mathrm{H}$,
$\left.\mathrm{OCH}_{3}\right), 3.80\left(s, 3 \mathrm{H}, \mathrm{OCH}_{3}\right), 5.40(d, 1 \mathrm{H}, J=10.8 \mathrm{~Hz}, \mathrm{C}(\mathrm{O}) \mathrm{CHC}(\mathrm{O}))$, $5.74(t, 1 \mathrm{H}, J=10.1 \mathrm{~Hz}, \mathrm{NHCH}), 6.72(d, 1 \mathrm{H}, J=15.7 \mathrm{~Hz}), 6.86(d$, $1 \mathrm{H}, J=15.7 \mathrm{~Hz}), 6.94-6.99(m, 1 \mathrm{H}), 7.02-7.08(m, 2 \mathrm{H}), 7.02-$ $7.08(m, 2 \mathrm{H}), 7.09-7.18(m, 2 \mathrm{H}), 7.25(t, 2 \mathrm{H}, J=7.5 \mathrm{~Hz}), 7.30(d$, $2 \mathrm{H}, J=10.4 \mathrm{~Hz}), 7.36(d, 1 \mathrm{H}, J=7.5 \mathrm{~Hz}), 7.55(d, 1 \mathrm{H}, J=15.7$ $\mathrm{Hz}), 8.39(d, 1 \mathrm{H}, \mathrm{C}(\mathrm{O}) \mathrm{NH}, J=9.0 \mathrm{~Hz}), 9.19(\mathrm{~s}, 1 \mathrm{H}, \mathrm{OH}) ; 9.24(\mathrm{~s}$, $1 \mathrm{H}, \mathrm{OH}) ;{ }^{13} \mathrm{C}$ NMR $\left(125.76 \mathrm{MHz}, \mathrm{DMSO}-d_{6}\right): \delta 22.6,51.8,55.6$, 67.6, 112.0, 112.1, 114.1, 114.2, 114.3, 122.2, 122.3, 122.6, $126.7,127.1,127.5,128.1,140.4,141.3,144.0,146.7,146.0$, 150.6, 168.1, 192.0, 192.3; IR (FTIR, $\mathrm{cm}^{-1}$ ): 982, 1020, 1072, 1130, 1269, 1439, 1512, 1578 and 3325; (ES, neg. mode) $m / z=514.1[\mathrm{M}-\mathrm{H}]^{-}$.

$N-((E)-5-(4-H y d r o x y-3-m e t h o x y p h e n y l)-2-((E)-3-(4-$ hydroxy-3-methoxyphenyl)acryloyl)-3-oxo-1-(4(trifluoromethyl)phenylpent-4-en-1-yl)acetamide (24) $\mathrm{C}_{31} \mathrm{H}_{28} \mathrm{~F}_{3} \mathrm{NO}_{7} ; \mathrm{M}=583.2$; yield: $42 \%$; m.p.: $144-147^{\circ} \mathrm{C} ;{ }^{1} \mathrm{H}$ NMR $\left(500.13 \mathrm{MHz}, \mathrm{DMSO}-d_{6}\right)$ : $\delta 1.74\left(s, 3 \mathrm{H}, \mathrm{CH}_{3} \mathrm{C}(\mathrm{O})\right), 3.74(s, 3 \mathrm{H}$, $\left.\mathrm{OCH}_{3}\right), 3.82\left(\mathrm{~s}, 3 \mathrm{H}, \mathrm{OCH}_{3}\right), 5.15(d, 1 \mathrm{H}, J=10.9 \mathrm{~Hz}, \mathrm{C}(\mathrm{O}) \mathrm{CHC}(\mathrm{O}))$, $5.79(t, 1 \mathrm{H}, J=9.7 \mathrm{~Hz}, \mathrm{NHCH}), 6.77(d, 1 \mathrm{H}, J=8.0 \mathrm{~Hz}), 6.83(d$, $1 \mathrm{H}, J=7.6 \mathrm{~Hz}), 6.88(d, 1 \mathrm{H}, J=15.2 \mathrm{~Hz}), 6.96(d, 1 \mathrm{H}, J=17.3$ $\mathrm{Hz}), 7.10(d, 1 \mathrm{H}, J=8.0 \mathrm{~Hz}), 7.17-7.27(m, 2 \mathrm{H}), 7.33(s, 1 \mathrm{H}), 7.39$ $(d, 1 \mathrm{H}, J=15.2 \mathrm{~Hz}), 7.57-7.71(m, 5 \mathrm{H}), 8.50(d, 1 \mathrm{H}, J=8.8 \mathrm{~Hz}$, $\mathrm{C}(\mathrm{O}) \mathrm{NH}), 9.74$ (bs, 2H, 2xOH); ${ }^{13} \mathrm{C}$ NMR $(125.76 \mathrm{MHz}$, DMSO$\left.d_{6}\right): \delta 22.6,51.5,55.6,55.7,66.6,111.6,115.6,115.7,121.9$, $122.4,123.7,124.0,124.9,125.0,125.3,125.6,128.3,144.6$, 144.7, 146.1, 147.8, 147.9, 149.9, 150.0, 168.3, 191.5, 191.8; IR $\left(F T I R, \mathrm{~cm}^{-1}\right)$ : 1070, 1124, 1165, 1279, 1327, 1520, 1574, 1647 and 3460; (ES, neg. mode) $\mathrm{m} / \mathrm{z}=582.2[\mathrm{M}-\mathrm{H}]^{-}$.

4-((E)-1-Acetamido-5-(4-hydroxy-3-methoxyphenyl)-2((E)-3-(4-hydroxy-3-methoxyphenyl)acryloyl)-3-oxopent-4en-1-yl)benzoic acid (25)

$\mathrm{C}_{31} \mathrm{H}_{29} \mathrm{NO}_{9}, \mathrm{M}=559.2$; yield: $48 \%$; m.p.: $199-201{ }^{\circ} \mathrm{C} ;{ }^{1} \mathrm{H}$ NMR $\left(500.13 \mathrm{MHz}, \mathrm{DMSO}-d_{6}\right): \delta 1.72\left(s, 3 \mathrm{H}, \mathrm{CH}_{3} \mathrm{C}(\mathrm{O})\right), 3.77(\mathrm{~s}, 3 \mathrm{H}$, $\left.\mathrm{OCH}_{3}\right), 3.82\left(s, 3 \mathrm{H}, \mathrm{OCH}_{3}\right), 5.14(d, 1 \mathrm{H}, J=10.6 \mathrm{~Hz}, \mathrm{C}(\mathrm{O}) \mathrm{CHC}(\mathrm{O}))$, $5.78(t, 1 \mathrm{H}, J=10.0 \mathrm{~Hz}, \mathrm{NHCH}), 6.78(d, 1 \mathrm{H}, J=8.2 \mathrm{~Hz}), 6.81-$ $6.89(m, 2 \mathrm{H}), 6.97(d, 1 \mathrm{H}, J=16.0 \mathrm{~Hz}), 7.09(d d$, overlapped peaks, $1 \mathrm{H}, J=8.2 \mathrm{~Hz}), 7.18-7.24(\mathrm{~m}, 2 \mathrm{H}), 7.33(\mathrm{bs}, 1 \mathrm{H}), 7.38(d$, $1 \mathrm{H}, J=15.7 \mathrm{~Hz}), 7.50(d, 2 \mathrm{H}, J=8.2 \mathrm{~Hz}), 7.65(d, 1 \mathrm{H}, J=16.0$ $\mathrm{Hz}), 7.84(d, 2 \mathrm{H}, J=8.2 \mathrm{~Hz}), 8.50(d, 1 \mathrm{H}, J=9.0 \mathrm{~Hz}, \mathrm{C}(\mathrm{O}) \mathrm{NH})$, 9.80 (bs, $2 \mathrm{H}, 2 \times \mathrm{OH}) ;{ }^{13} \mathrm{C}$ NMR $\left(125.76 \mathrm{MHz}, \mathrm{DMSO}-d_{6}\right): \delta 22.6$, 51.6, 55.6, 55.7, 66.7, 111.5, 111.6, 115.6, 115.7, 122.0, 122.5, 123.7, 124.0, 125.3, 125.7, 127.6, 129.1, 144.5, 144.6, 146.1, 147.9, 148.0, 150.0, 150.1, 167.1, 168.3, 191.6, 191.9; IR (FTIR, $\mathrm{cm}^{-1}$ ): 982, 1032, 1124, 1167, 1273, 1429, 1514, 1579, 1647, 2357 and 3396; (ES, neg. mode) $\mathrm{m} / \mathrm{z}=558.2[\mathrm{M}-\mathrm{H}]^{-}$.

N-((E)-5-(4-Hydroxy-3-methoxyphenyl)-2-((E)-3-(4hydroxy-3-methoxyphenyl)acryloyl)-3-oxo-1-phenylpent4-en-1-yl)acetamide (26)

$\mathrm{C}_{30} \mathrm{H}_{29} \mathrm{NO}_{7} ; \mathrm{M}=515.2$; yield: $40 \%$; m.p.: $145-147^{\circ} \mathrm{C} ;{ }^{1} \mathrm{H} \mathrm{NMR}$ $\left(500.13 \mathrm{MHz}, \mathrm{CDCl}_{3}\right): \delta 1.97\left(\mathrm{~s}, 3 \mathrm{H}, \mathrm{CH}_{3} \mathrm{C}(\mathrm{O})\right), 3.89$ (bs, 6H, $\left.\mathrm{OCH}_{3}\right), 4.78(d, 1 \mathrm{H}, J=6.5 \mathrm{~Hz}, \mathrm{C}(\mathrm{O}) \mathrm{CHC}(\mathrm{O})), 6.02(t, 1 \mathrm{H}$, $J=8.2 \mathrm{~Hz}, \mathrm{CHNH}), 6.22(s, 1 \mathrm{H}, \mathrm{OH}), 6.30(\mathrm{~s}, 1 \mathrm{H}, \mathrm{OH}), 6.70(t$, $2 \mathrm{H}, J=16.9 \mathrm{~Hz}), 6.87(t, 2 \mathrm{H}, J=8.9 \mathrm{~Hz}), 6.97-7.04(m, 3 \mathrm{H}), 7.06$ 
$(d, 1 \mathrm{H}, J=8.4 \mathrm{~Hz}), 7.10(d, 1 \mathrm{H}, J=9.1 \mathrm{~Hz}), 7.18-7.24(m, 1 \mathrm{H})$, $7.29(t, 2 \mathrm{H}, J=7.3 \mathrm{~Hz}), 7.35-7.41(m, 1 \mathrm{H}), 7.50(d, 1 \mathrm{H}$, $J=15.7 \mathrm{~Hz}), 7.59(d, 1 \mathrm{H}, J=15.7 \mathrm{~Hz}) ;{ }^{13} \mathrm{C} \mathrm{NMR}(125.76 \mathrm{MHz}$, $\left.\mathrm{CDCl}_{3}\right): \delta 22.9,51.9,55.5,66.6,109.3,109.5,114.4,114.5$, $121.0,121.5,123.9,124.2,126.0,126.3,127.2,128.2,139.6$, 145.0, 145.1, 146.5, 148.6, 169.2, 192.2, 194.7; IR (FTIR, cm ${ }^{-1}$ ): $979,1032,1122,1163,1269,1429,1514,1579,1655,2362$ and 3390; (ES, neg. mode) $m / z=514.2[\mathrm{M}-\mathrm{H}]^{-}$.

$N-((E)-1-(3,4-D i f l u o r o p h e n y l)-5-(4-h y d r o x y-3-$

methoxyphenyl)-2-((E)-3-(4-hydroxy-3-methoxyphenyl)acryloyl)-3-oxopent-4-en-1-yl)acetamide (27)

$\mathrm{C}_{30} \mathrm{H}_{27} \mathrm{~F}_{2} \mathrm{NO}_{7} ; \mathrm{M}=551.2$; yield: $40 \%$; m.p.: $163-166^{\circ} \mathrm{C} ;{ }^{1} \mathrm{H}$ NMR (500.13 MHz, DMSO- $\left.d_{6}\right): \delta 1.73\left(s, 3 \mathrm{H}, \mathrm{CH}_{3} \mathrm{C}(\mathrm{O})\right), 3.78(s, 3 \mathrm{H}$, $\left.\mathrm{OCH}_{3}\right), 3.82(\mathrm{~s}, 3 \mathrm{H}, \mathrm{OCH})_{3}, 5.09(d, 1 \mathrm{H}, J=10.4 \mathrm{~Hz}, \mathrm{C}(\mathrm{O}) \mathrm{CHC}(\mathrm{O}))$, $5.71(t, 1 \mathrm{H}, J=9.5 \mathrm{~Hz}, \mathrm{NHCH}), 6.78(d, 1 \mathrm{H}, J=7.6 \mathrm{~Hz}), 6.83(d$, $1 \mathrm{H}, J=8.0 \mathrm{~Hz}), 6.90(d d$, overlapped peaks, $2 \mathrm{H}, J=16.5 \mathrm{~Hz})$, $7.11(d, 1 \mathrm{H}, J=7.6 \mathrm{~Hz}), 7.17-7.27(m, 3 \mathrm{H}), 7.32(\mathrm{bs}, 2 \mathrm{H}), 7.40(d$, $1 \mathrm{H}, J=16.1 \mathrm{~Hz}), 7.67(d, 1 \mathrm{H}, J=15.8 \mathrm{~Hz}), 8.41(d, 1 \mathrm{H}, \mathrm{C}(\mathrm{O}) \mathrm{NH}$, $J=8.2 \mathrm{~Hz}), 9.71$ (bs, $2 \mathrm{H}, 2 \times \mathrm{OH}) ;{ }^{13} \mathrm{C}$ NMR $(125.76 \mathrm{MHz}$, DMSO- $\left.d_{6}\right): \delta 22.6,51.0,55.6,55.7,66.7,111.6,115.6,115.7$, $116.3,116.4,116.9,117.1,121.9,122.5,123.7,124.0,125.3$, $125.6,139.2,144.6,144.8,147.9,148.0,149.9,150.1,168.3$, 191.6, 191.8; IR (FTIR, $\mathrm{cm}^{-1}$ ): 987, 1124, 1211, 1263, 1419, 1516, 1587, 1622, 2366 and 3442; (ES, neg. mode) $\mathrm{m} / \mathrm{z}=550.2$ $[\mathrm{M}-\mathrm{H}]^{-}$.

$N-((E)-5-(3-H y d r o x y p h e n y l)-2-((E)-3-(3-h y d r o x y p h e n y l)-$ acryloyl)-3-oxo-1-phenylpent-4-en-1-yl)acetamide (28) $\mathrm{C}_{28} \mathrm{H}_{25} \mathrm{NO}_{5}$; 455.2; yield: $51 \%$; m.p.: $200-202^{\circ} \mathrm{C}$; ${ }^{1} \mathrm{H}$ NMR $\left(500.13 \mathrm{MHz}, \mathrm{DMSO}-d_{6}\right): \delta 1.70\left(\mathrm{~s}, 3 \mathrm{H}, \mathrm{C}(\mathrm{O}) \mathrm{CH}_{3}\right), 5.14(d, 1 \mathrm{H}$, $J=10.7 \mathrm{~Hz}, \mathrm{C}(\mathrm{O}) \mathrm{CHC}(\mathrm{O})), 5.77(t, 1 \mathrm{H}, J=10.1 \mathrm{~Hz}, \mathrm{NHCH}), 6.77-$ $6.93(m, 3 \mathrm{H}), 7.00(d, 1 \mathrm{H}, J=9.7 \mathrm{~Hz}), 7.05(d, 1 \mathrm{H}, J=10.6 \mathrm{~Hz})$, 7.08 (bs, 1H), 7.13-7.21 (m, 4H), 7.22-7.30 (m, 3H), 7.32 (s, 1H), $7.37(t, 2 \mathrm{H}, J=5.3$ and $7.6 \mathrm{~Hz}), 7.60(d, 1 \mathrm{H}, J=15.9 \mathrm{~Hz}), 8.42(d$, $1 \mathrm{H}, J=9.4 \mathrm{~Hz}), 9.61(s, 1 \mathrm{H}, \mathrm{OH}), 9.66(s, 1 \mathrm{H}, \mathrm{OH}) ;{ }^{13} \mathrm{C} N M R$ $\left(125.76 \mathrm{MHz}\right.$, DMSO- $\left.d_{6}\right): \delta 22.7,51.9,67.4,114.8,114.9,118.2$, $118.3,119.9,125.1,125.2,127.2,127.5,128.2,130.0,130.1$, 135.2, 135.5, 141.1, 143.9, 157.7, 157.8, 168.2, 192.4 and 192.7; IR (FTIR, $\left.\mathrm{cm}^{-1}\right)$ : 980, 1072, 1173, 1234, 1267, 1452, 1545, 1645 and 3369; MS (ES, neg. mode) $\mathrm{m} / \mathrm{z}=454.2[\mathrm{M}-\mathrm{H}]^{-}$.

$\mathrm{N}-((E)-5-(4-B r o m o t h i o p h e n-2-y l)-2-((E)-3-(4-$

bromothiophen-2-yl)acryloyl)-3-oxo-1-phenylpent-4-en-1yl)acetamide (29)

$\mathrm{C}_{24} \mathrm{H}_{19} \mathrm{Br}_{2} \mathrm{NO}_{3} \mathrm{~S}_{2} ; \mathrm{M}=590.9$; yield: $39 \%$; m.p.: $148-150^{\circ} \mathrm{C}$; ${ }^{1} \mathrm{H}$ NMR $\left(500.13 \mathrm{MHz}, \mathrm{DMSO}-d_{6}\right): \delta 1.71\left(s, 3 \mathrm{H}, \mathrm{C}(\mathrm{O}) \mathrm{CH}_{3}\right)$, $5.07(d, 1 \mathrm{H}, \mathrm{C}(\mathrm{O}) \mathrm{CHC}(\mathrm{O}), J=10.7 \mathrm{~Hz}), 5.69(t, 1 \mathrm{H}, \mathrm{NHCH}$, $J=10.3 \mathrm{~Hz}), 6.75(d, 1 \mathrm{H}, J=15.7 \mathrm{~Hz}), 6.89(d, 1 \mathrm{H}, J=15.8 \mathrm{~Hz})$, 7.16-7.22 $(m, 1 \mathrm{H}), 7.27(t, 2 \mathrm{H}, J=7.5 \mathrm{~Hz}), 7.36(d, 2 \mathrm{H}$, $J=7.5 \mathrm{~Hz}), 7.52(d, 1 \mathrm{H}, J=15.4 \mathrm{~Hz}), 7.59(s, 1 \mathrm{H}), 7.64(s, 1 \mathrm{H})$, $7.80(d, 1 \mathrm{H}, J=5.8 \mathrm{~Hz}), 7.84(\mathrm{~s}, 1 \mathrm{H}), 7.88(\mathrm{~s}, 1 \mathrm{H}), 8.41(\mathrm{~d}, 1 \mathrm{H}$, $J=8.8 \mathrm{~Hz}) .{ }^{13} \mathrm{C}$ NMR $\left(125.76 \mathrm{MHz}\right.$, DMSO- $\left.d_{6}\right): \delta 23.4,48.1,56.5$, $67.5,108.5,111.5,112.5,112.6,123.5,123.9,124.3,124.6$, $124.9,127.6,127.8,128.0,145.4,145.5,147.6,149.86,149.89$, 152.4, 152.5, 169.4, 192.3; IR (FTIR, $\left.\mathrm{cm}^{-1}\right): 578,696,825,954$,
1070, 1290, 1392, 1502, 1593, 1980, 2177 and 3277; MS (ES, neg. mode) $\mathrm{m} / \mathrm{z}=589.9[\mathrm{M}-\mathrm{H}]^{-}$.

$N-((E)-5-(3,5-D i h y d r o x y p h e n y l)-2-((E)-3-(3,5-$ dihydroxyphenyl)acryloyl)-3-oxo-1-phenylpent-4-en-1-yl)acetamide (30)

$\mathrm{C}_{28} \mathrm{H}_{25} \mathrm{NO}_{7} ; \mathrm{M}=487.16$; yield: $35 \%$; m.p.: $145-148^{\circ} \mathrm{C} ;{ }^{1} \mathrm{H}$ NMR $\left(500.13 \mathrm{MHz}, \mathrm{DMSO}-d_{6}\right): \delta 1.71\left(\mathrm{~s}, 3 \mathrm{H}, \mathrm{C}(\mathrm{O}) \mathrm{CH}_{3}\right), 5.09(d, 1 \mathrm{H}$, $J=11.3 \mathrm{~Hz}, \mathrm{C}(\mathrm{O}) \mathrm{CHC}(\mathrm{O})), 5.75(t, 1 \mathrm{H}, J=9.1 \mathrm{~Hz}, \mathrm{NHCH}), 6.31(d$, $2 \mathrm{H}, J=16.9 \mathrm{~Hz}), 6.45(s, 2 \mathrm{H}), 6.55(s, 2 \mathrm{H}), 6.74(d, 1 \mathrm{H}$, $J=15.9 \mathrm{~Hz}), 6.91(d, 1 \mathrm{H}, J=15.9 \mathrm{~Hz}), 7.18(t, 1 \mathrm{H}, J=7.5 \mathrm{~Hz})$, 7.24-7.30 $(m, 3 \mathrm{H}), 7.37(d, 2 \mathrm{H}, J=7.9 \mathrm{~Hz}), 7.47(d, 1 \mathrm{H}$, $J=15.8 \mathrm{~Hz}), 8.40(d, 1 \mathrm{H}, J=8.9 \mathrm{~Hz}), 9.45(2 \mathrm{H}, 2 \times \mathrm{OH})$ and $9.49(2 \mathrm{H} 2 \times \mathrm{OH}) ;{ }^{13} \mathrm{C}$ NMR $\left(125.76 \mathrm{MHz}, \mathrm{DMSO}-d_{6}\right): \delta 22.9$, $51.8,64.9,105.4,105.5,106.6,124.7,124.8,127.2,127.5,128.1$, $135.5,135.8,141.0,144.1,144.2,158.7,158.8,168.1,192.3$ and 192.6; IR (FTIR, $\left.\mathrm{cm}^{-1}\right): 516,603,669,839,960,1147,1300$, $1598,1975,2164$ and 3209; MS (ES, neg. mode) $\mathrm{m} / \mathrm{z}=486.2$ $[\mathrm{M}-\mathrm{H}]^{-}$.

$\mathrm{N}-((E)-5-(4-H y d r o x y p h e n y l)-2-((E)-3-(4-h y d r o x y p h e n y l)-$ acryloyl)-3-oxo-1-phenylpent-4-en-1-yl)acetamide (31) $\mathrm{C}_{28} \mathrm{H}_{25} \mathrm{NO}_{5} ; \mathrm{M}=455.17$; yield: $47 \%$; m.p.: $179-182^{\circ} \mathrm{C} .{ }^{1} \mathrm{H}$ NMR $\left(500.13 \mathrm{MHz}, \mathrm{DMSO}-d_{6}\right): \delta 1.70\left(\mathrm{~s}, 3 \mathrm{H}, \mathrm{C}(\mathrm{O}) \mathrm{CH}_{3}\right), 5.01(d, 1 \mathrm{H}$, $J=10.2 \mathrm{~Hz}, \mathrm{C}(\mathrm{O}) \mathrm{CHC}(\mathrm{O})), 5.76(t, 1 \mathrm{H}, J=9.3 \mathrm{~Hz}, \mathrm{NHCH}), 6.74-$ $6.80(m, 3 \mathrm{H}), 6.83(d, 2 \mathrm{H}, J=8.6 \mathrm{~Hz}), 6.91(d, 1 \mathrm{H}, J=15.7 \mathrm{~Hz})$, $7.16(t, 1 \mathrm{H}, J=7.3 \mathrm{~Hz}), 7.27(t, 2 \mathrm{H}, J=7.6 \mathrm{~Hz}), 7.33-7.40(m$, $3 \mathrm{H}), 7.50(d, 2 \mathrm{H}, J=8.6 \mathrm{~Hz}), 7.58(d, 2 \mathrm{H}, J=8.6 \mathrm{~Hz}), 7.62(s, 1 \mathrm{H})$, $8.39(d, 1 \mathrm{H}, J=9.1 \mathrm{~Hz}), 10.11(s, 2 \mathrm{H}, 2 \times \mathrm{OH}) ;{ }^{13} \mathrm{C} \mathrm{NMR}$ $\left(125.76 \mathrm{MHz}\right.$, DMSO- $\left.d_{6}\right): \delta 22.6,51.8,67.6,115.8,115.9$, $121.9,124.9,125.2,127.0,127.5,128.0,130.8,130.9,141.3$, 143.8, 143.9, 160.3, 160.4, 168.1, 191.9 and 192.2; IR (FTIR, $\mathrm{cm}^{-1}$ ): 978, 1092, 1171, 1275, 1439, 1513, 1578, 1601, 1641, 2355 and 3408; MS (ES, neg. mode) $\mathrm{m} / \mathrm{z}=454.2[\mathrm{M}-\mathrm{H}]^{-}$.

$N-((E)-5-(4-F l u o r o p h e n y l)-2-((E)-3-(4-f l u o r o p h e n y l)-$ acryloyl)-3-oxo-1-phenylpent-4-en-1-yl)acetamide (32) $\mathrm{C}_{28} \mathrm{H}_{23} \mathrm{~F}_{2} \mathrm{NO}_{3} ; \mathrm{M}=459.16$; yield: $56 \%$; m.p.: $209-212^{\circ} \mathrm{C}$. ${ }^{1} \mathrm{H}$ NMR $\left(500.13 \mathrm{MHz}, \mathrm{DMSO}-d_{6}\right): \delta 1.70\left(\mathrm{~s}, 3 \mathrm{H}, \mathrm{C}(\mathrm{O}) \mathrm{CH}_{3}\right)$, $5.14(d, 1 \mathrm{H}, J=10.8 \mathrm{~Hz}, \mathrm{C}(\mathrm{O}) \mathrm{CHC}(\mathrm{O})), 5.78(t, 1 \mathrm{H}, J=9.5 \mathrm{~Hz}$, $\mathrm{NHCH}), 6.98(d, 1 \mathrm{H}, J=16.2 \mathrm{~Hz}), 7.10(d, 1 \mathrm{H}, J=15.8 \mathrm{~Hz}), 7.17$ $(t, 1 \mathrm{H}, J=7.1 \mathrm{~Hz}), 7.21-7.34(m, 6 \mathrm{H}), 7.39(d, 2 \mathrm{H}, J=8.1 \mathrm{~Hz})$, $7.44(d, 1 \mathrm{H}, J=16.2 \mathrm{~Hz}), 7.69(s, 1 \mathrm{H}), 7.71-7.76(m, 2 \mathrm{H}), 7.82(t$, $2 \mathrm{H}, J=8.1 \mathrm{~Hz}), 8.42(d, 1 \mathrm{H}, J=8.6 \mathrm{~Hz}) ;{ }^{13} \mathrm{C}$ NMR $(125.76 \mathrm{MHz}$, DMSO- $\left.d_{6}\right): \delta 22.6,51.8,67.3,115.9(d), 116.1(d), 125.1,125.3$, $127.2,127.4\left(2 \times \mathrm{CH}_{\mathrm{Ar}}\right), 128.1\left(2 \times \mathrm{CH}_{\mathrm{Ar}}\right), 130.6(\mathrm{~m}), 130.9$, $131.0(d), 131.1(d), 141.0,142.4\left(2 \times \mathrm{CH}_{\mathrm{Ar}}\right), 162.5,164.5,168.1$, 192.2 and 192.5; IR (FTIR, $\mathrm{cm}^{-1}$ ): 980, 1092, 1161, 1238, 1511, 1597, 1658, 1678 and 3313; MS (ES, neg. mode) $\mathrm{m} / \mathrm{z}=458.2$ $[\mathrm{M}-\mathrm{H}]^{-}$.

4,4'-((E)-5-Acetamido-4-((E)-3-(4-carboxyphenyl)acryloyl)3-oxopent-1-ene-1,5-diyl)dibenzoic acid (33)

$\mathrm{C}_{31} \mathrm{H}_{25} \mathrm{NO}_{9} ; \mathrm{M}=555.18$; yield: $49 \%$; m.p.: $220^{\circ} \mathrm{C}$ (decomposed). ${ }^{1} \mathrm{H}$ NMR $\left(500.13 \mathrm{MHz}\right.$, DMSO- $\left.d_{6}\right): \delta 1.71(s, 3 \mathrm{H}), 5.29(d, 1 \mathrm{H}$, $J=10.7 \mathrm{~Hz}), 5.80(t, 1 \mathrm{H}, J=9.7 \mathrm{~Hz}), 7.14(d, 1 \mathrm{H}, J=15.7 \mathrm{~Hz})$, 
$7.24(d, 1 \mathrm{H}, J=16.8 \mathrm{~Hz}), 7.45-7.55(m, 3 \mathrm{H}), 7.60(d, 1 \mathrm{H}$, $J=8.1 \mathrm{~Hz}), 7.72-7.80(m, 3 \mathrm{H}), 7.82-7.88(m, 3 \mathrm{H}), 7.91(d, 2 \mathrm{H}$, $J=7.7 \mathrm{~Hz}), 7.98(d, 2 \mathrm{H}, J=8.2 \mathrm{~Hz}), 8.52(d, 1 \mathrm{H}, J=9.0 \mathrm{~Hz})$; ${ }^{13} \mathrm{C}$ NMR $\left(125.76 \mathrm{MHz}\right.$, DMSO- $\left.d_{6}\right): \delta 22.6,51.7,66.8,127.1$, $127.6,127.7,128.8,128.9,129.3,129.7,129.8,132.4,137.9$, 138.3, 142.4, 142.5, 145.8, 166.7, 166.8, 167.0, 168.4, 192.3, 192.6; IR (FTIR, cm ${ }^{-1}$ ): 541, 781, 1234, 1610, 1695 and 2164; MS (ES, neg. mode) $\mathrm{m} / \mathrm{z}=554.2[\mathrm{M}-\mathrm{H}]^{-}$.

4-((4E,6E)-1-Acetamido-7-(4-hydroxy-3-methoxyphenyl)2-((2E,4E)-5-(4-hydroxy-3-methoxyphenyl)penta-2,4dienoyl)-3-oxohepta-4,6-dienyl)benzoic acid (34)

$\mathrm{C}_{35} \mathrm{H}_{33} \mathrm{NO}_{9} ; \mathrm{M}=611.17$; yield: $39 \%$; m.p.: $137-139^{\circ} \mathrm{C} .{ }^{1} \mathrm{H}$ NMR $\left(500.13 \mathrm{MHz}, \mathrm{DMSO}-d_{6}\right): \delta 1.72(s, 3 \mathrm{H}), 3.78(s, 3 \mathrm{H}), 3.81(s, 3 \mathrm{H})$, $4.91(d, 1 \mathrm{H}, J=10.6 \mathrm{~Hz}), 5.91(t, 1 \mathrm{H}, J=9.7 \mathrm{~Hz}), 6.31(d, 1 \mathrm{H}$, $J=15.8 \mathrm{~Hz}), 6.44(d, 1 \mathrm{H}, J=14.8 \mathrm{~Hz}), 6.70-7.26(m, 11 \mathrm{H}), 7.41-$ $7.51(m, 3 \mathrm{H}), 7.82-7.88(m, 2 \mathrm{H}), 8.45(d, 2 \mathrm{H}, J=9.5 \mathrm{~Hz}), 9.51$ (bs, $2 \mathrm{H}) ;{ }^{13} \mathrm{C}$ NMR $\left(125.76 \mathrm{MHz}\right.$, DMSO- $\left.d_{6}\right): \delta 23.5,52.6,56.5,56.5$, $68.1,111.2,116.5,123.1,123.2,124.6,124.8,127.0,127.1$, 128.36, 128.43, 128.6, 130.1, 130.5, 144.6, 144.7, 146.2, 146.3, 147.0, 148.8, 148.8, 149.5, 167.9, 169.1, 192.5, 192.7; IR (FTIR, $\mathrm{Cm}^{-1}$ ): 995, 1032, 1124, 1284, 1514, 1572, 1653, 2357 and 3367; MS (ES, neg. mode) $m / z=610.2[\mathrm{M}-\mathrm{H}]^{-}$.

$N-((4 E, 6 E)-7-(4-H y d r o x y-3-m e t h o x y p h e n y l)-2-((2 E, 4 E)-5-$ (4-hydroxy-3-methoxyphenyl)penta-2,4-dienoyl)-3-oxo-1phenylhepta-4,6-dienyl)acetamide (35)

$\mathrm{C}_{34} \mathrm{H}_{33} \mathrm{NO}_{7} ; \mathrm{M}=567.12$; yield: $41 \%$; m.p.: $125^{\circ} \mathrm{C}$ (decomposed). ${ }^{1} \mathrm{H}$ NMR $\left(500.13 \mathrm{MHz}\right.$, DMSO- $\left.d_{6}\right): \delta 1.71(s, 3 \mathrm{H}), 3.78(s, 3 \mathrm{H})$, $3.81(s, 3 \mathrm{H}), 4.83(d, 1 \mathrm{H}, J=11.1 \mathrm{~Hz}), 5.82(t, 1 \mathrm{H}, J=9.8 \mathrm{~Hz}), 6.29$ $(d, 1 \mathrm{H}, J=15.5 \mathrm{~Hz}), 6.45(d, 1 \mathrm{H}, J=15.2 \mathrm{~Hz}), 6.72-6.80(m, 3 \mathrm{H})$, 6.84-7.39 $(m, 14 \mathrm{H}), 8.37(d, 1 \mathrm{H}, J=7.3 \mathrm{~Hz}), 9.50(s, 2 \mathrm{H})$; ${ }^{13} \mathrm{C}$ NMR $\left(125.76 \mathrm{MHz}\right.$, DMSO- $\left.d_{6}\right): \delta 23.5,52.7,56.5,56.5,68.7$, $111.2,116.5,123.1,123.1,124.7,124.8,126.9,127.3,128.0$, $128.4,128.5,129.0,142.1,144.4,144.5,146.0,146.0,148.8$, 148.8, 149.5, 168.9, 192.7, 193.0; IR (FTIR, $\mathrm{cm}^{-1}$ ): 1001, 1030, 1120, 1161, 1282, 1514, 1566, 1655, 2362 and 3313; MS (ES, neg. mode) $\mathrm{m} / \mathrm{z}=566.1[\mathrm{M}-\mathrm{H}]^{-}$.

$N-((E)-5-(5-(H y d r o x y m e t h y l) f u r a n-2-y l)-2-((E)-3-(5-$ (hydroxymethyl)furan-2-yl)acryloyl)-3-oxo-1-phenylpent4-en-1-yl)acetamide (36)

$\mathrm{C}_{26} \mathrm{H}_{25} \mathrm{NO}_{7} ; \mathrm{M}=463.16$; yield: $38 \%$; m.p.: $129-132^{\circ} \mathrm{C} ;{ }^{1} \mathrm{H} \mathrm{NMR}$ (500.13 MHz, DMSO- $\left.d_{6}\right): \delta 1.70\left(s, 3 \mathrm{H}, \mathrm{C}(\mathrm{O}) \mathrm{CH}_{3}\right), 4.45(d, 4 \mathrm{H}$, $J=16.8 \mathrm{~Hz}), 4.96(d, 1 \mathrm{H}, J=10.7 \mathrm{~Hz}, \mathrm{C}(\mathrm{O}) \mathrm{CHC}(\mathrm{O})), 5.41$ (bs, $2 \mathrm{H}$, $2 \times \mathrm{OH}), 5.78(t, 1 \mathrm{H}, J=10.0 \mathrm{~Hz}, \mathrm{NHCH}), 6.45(d, 1 \mathrm{H}, J=2.2 \mathrm{~Hz})$, $6.49(d, 1 \mathrm{H}, J=2.2 \mathrm{~Hz}), 6.58(d, 1 \mathrm{H}, J=15.8 \mathrm{~Hz}), 6.74(d, 1 \mathrm{H}$, $J=15.7 \mathrm{~Hz}), 6.92(d, 1 \mathrm{H}, J=2.2 \mathrm{~Hz}), 6.99(d, 1 \mathrm{H}, J=2.2 \mathrm{~Hz})$, 7.15-7.30 (m, 4H), $7.35(d, 2 \mathrm{H}, J=7.2 \mathrm{~Hz}), 7.45(d, 1 \mathrm{H}$, $J=15.7 \mathrm{~Hz}), 8.39\left((d, 1 \mathrm{H}, J=9.1 \mathrm{~Hz}) ;{ }^{13} \mathrm{C} \mathrm{NMR}(125.76 \mathrm{MHz}\right.$, $\left.\mathrm{CDCl}_{3}\right): \delta 22.9,51.9,55.7,55.8,68.2,110.2,110.3,119.3,119.5$, $120.5,120.8,127.1,127.4\left(2 \times \mathrm{CH}_{\mathrm{Ar}}\right), 128.1\left(2 \times \mathrm{CH}_{\mathrm{Ar}}\right), 129.9$, 130.1, 140.1, 149.5, 149.8, 159.4, 159.6, 168.1, 191.4, 191.9; IR (FTIR, $\mathrm{cm}^{-1}$ ): 541, 698, 964, 1016, 1599, 2019, 2166 and 3298; MS (ES, neg. mode) $\mathrm{m} / \mathrm{z}=462.2[\mathrm{M}-\mathrm{H}]^{-}$.
$N-((E)-5-(3,4-D i m e t h o x y p h e n y l)-2-((E)-3-(3,4-$

dimethoxyphenyl)acryloyl)-3-oxo-1-phenylpent-4-en-1-yl)acrylamide (37)

$\mathrm{C}_{33} \mathrm{H}_{33} \mathrm{NO}_{7} ; \mathrm{M}=555.2$; yield: $57 \%$; m.p.: $186-189^{\circ} \mathrm{C} ;{ }^{1} \mathrm{H}$ NMR $\left(500.13 \mathrm{MHz} \mathrm{CDCl}_{3}\right): \delta 3.88$ (bs, $\left.12 \mathrm{H}, 4 \times \mathrm{XCH}_{3}\right), 4.86(d, 1 \mathrm{H}$, $J=6.3 \mathrm{~Hz}, \mathrm{C}(\mathrm{O}) \mathrm{CHC}(\mathrm{O})), 7.06-7.16(m, 2 \mathrm{H}) 6.21(d, 1 \mathrm{H}$, $J=17.1 \mathrm{~Hz}), 6.74(t, 2 \mathrm{H}, J=15.8 \mathrm{~Hz}), 6.79-6.87(m, 2 \mathrm{H})$ 6.99-7.04 $(m, 2 \mathrm{H}), 7.07(d, 1 \mathrm{H}, J=8.2 \mathrm{~Hz}), 7.10(d, 1 \mathrm{H}$, $J=8.2 \mathrm{~Hz}), 7.16-7.23(\mathrm{~m}, 1 \mathrm{H}), 7.28(t, 2 \mathrm{H}, J=7.6 \mathrm{~Hz}), 7.36(d$, $1 \mathrm{H}, J=9.0 \mathrm{~Hz}), 7.41(d, 2 \mathrm{H}, J=7.5 \mathrm{~Hz}), 7.52(d, 1 \mathrm{H}, J=15.8 \mathrm{~Hz})$, $7.61(d, 1 \mathrm{H}, J=15.8 \mathrm{~Hz}) ;{ }^{13} \mathrm{C}$ NMR $\left(125.76 \mathrm{MHz} \mathrm{CDCl}_{3}\right): \delta 52.1$, 55.5, 66.6, 109.6, 109.7, 110.5, 110.6, 121.3, 121.9, 123.5, $123.7,126.40,126.48,126.52,127.2,128.2,130.3,139.5$, $144.9,145.0,148.8,151.54,151.56,164.5,192.1,194.7$; IR $\left(F T I R, \mathrm{~cm}^{-1}\right.$ ): 545, 700, 759, 979, 1022, 1141, 1258, 1512, 1593, 1652, 1967, 2054 and 2166; MS (ES, neg. mode) $\mathrm{m} / \mathrm{z}=554.2$ $[\mathrm{M}-\mathrm{H}]^{-}$.

$N-((E)-5-(3,4-D i m e t h o x y p h e n y l)-2-((E)-3-(3,4-$

dimethoxyphenyl)acryloyl)-1-(4-fluorophenyl)-3-oxopent4-en-1-yl)acrylamide (38)

$\mathrm{C}_{33} \mathrm{H}_{32} \mathrm{FNO}_{7} ; \mathrm{M}=573.2$; yield: $55 \%$; ${ }^{1} \mathrm{H}$ NMR $(500.13 \mathrm{MHz}$, DMSO- $\left.d_{6}\right)$ : $\delta 3.76\left(s, 6 \mathrm{H}, 2 \times \mathrm{OCH}_{3}\right), 3.79\left(\mathrm{~s}, 3 \mathrm{H}, \mathrm{OCH}_{3}\right), 3.80(\mathrm{~s}$, $\left.3 \mathrm{H}, \mathrm{OCH}_{3}\right), 5.20(d, 1 \mathrm{H}, J=10.9 \mathrm{~Hz}, \mathrm{C}(\mathrm{O}) \mathrm{CHC}(\mathrm{O})), 5.52(d, 1 \mathrm{H}$, $J=10.2 \mathrm{~Hz}, \mathrm{CHH}=\mathrm{CH}), 5.82(t, 1 \mathrm{H}, J=9.3 \mathrm{~Hz}, \mathrm{CHNH}), 5.99(d$, $1 \mathrm{H}, J=16.8 \mathrm{~Hz}, \mathrm{CH}=\mathrm{CH}), 6.13(d d, 1 \mathrm{H}, J=10.2 \mathrm{~Hz}, \mathrm{CHH}=\mathrm{CH})$, 6.90-6.98 (m, 2H), 6.98-7.05 (m, 2H), 7.11 (bs, 3H), 7.17-7.27 $(m, 2 \mathrm{H}), 7.27-7.37(m, 2 \mathrm{H}), 7.38-7.49(m, 2 \mathrm{H}), 7.69(d, 1 \mathrm{H}$, $J=16.1 \mathrm{~Hz}), 8.66(d, 1 \mathrm{H}, J=8.6 \mathrm{~Hz}, \mathrm{C}(\mathrm{O}) \mathrm{NH}) ;{ }^{13} \mathrm{C} N \mathrm{NMR}$ $\left(125.76 \mathrm{MHz}\right.$, DMSO- $\left.d_{6}\right): \delta 51.3,55.6,67.0,110.6,111.6$, $111.7,114.8,115.0,123.0,123.4,123.7,123.9,125.7,126.7$, $126.9,129.5,129.6,131.5,137.4,144.3,144.4,148.9,149.0$, 151.5, 151.6, 163.7, 191.7, 192.0; IR (FTIR, cm ${ }^{-1}$ ): 980, 1022, 1142, 1263, 1512, 1589, 1649, 1674 and 3415; MS (ES, neg. mode) $m / z=572.2[\mathrm{M}-\mathrm{H}]^{-}$.

$N-((E)-5-(3,4-D i m e t h o x y p h e n y l)-2-((E)-3-(3,4-$

dimethoxyphenyl)acryloyl)-3-oxo-1-(3-(trifluoromethyl)phenyl)pent-4-en-1-yl)acrylamide (39)

$\mathrm{C}_{34} \mathrm{H}_{32} \mathrm{~F}_{3} \mathrm{NO}_{7} ; \mathrm{M}=623.2$; yield: $54 \%$; m.p.: $184-186^{\circ} \mathrm{C}$; ${ }^{1} \mathrm{H}$ NMR $\left(500.13 \mathrm{MHz}\right.$, DMSO- $\left.d_{6}\right): \delta 3.75\left(s, 3 \mathrm{H}, \mathrm{OCH}_{3}\right)$, $3.76\left(s, 3 \mathrm{H}, \mathrm{OCH}_{3}\right), 3.79\left(\mathrm{~s}, 3 \mathrm{H}, \mathrm{OCH}_{3}\right), 3.80\left(\mathrm{~s}, 3 \mathrm{H}, \mathrm{OCH}_{3}\right), 5.28$ $(d, 1 \mathrm{H}, J=10.8 \mathrm{~Hz}, \mathrm{C}(\mathrm{O}) \mathrm{CHC}(\mathrm{O})), 5.54(d, 1 \mathrm{H}, J=10.2 \mathrm{~Hz}$, $\mathrm{CHH}=\mathrm{CH}), 5.88(d d$, overlapped peaks, $1 \mathrm{H}, J=9.5 \mathrm{~Hz}$, $\mathrm{CHNH}), 6.01(d, 1 \mathrm{H}, J=17.2 \mathrm{~Hz}, \mathrm{CHHCH}), 6.16(d d, 1 \mathrm{H}$, $J=10.2 \mathrm{~Hz}, \mathrm{CHHCH}), 6.92-7.09(m, 5 \mathrm{H}), 7.20(d, 1 \mathrm{H}, J=8.3$ $\mathrm{Hz}), 7.24(s, 1 \mathrm{H}), 7.31(d, 1 \mathrm{H}, J=8.3 \mathrm{~Hz}), 7.35(s, 1 \mathrm{H}), 7.43(d$, $1 \mathrm{H}, J=15.7 \mathrm{~Hz}), 7.51-7.57(\mathrm{~m}, 2 \mathrm{H}), 7.69(s, 1 \mathrm{H}), 7.78(s, 1 \mathrm{H})$, $8.74\left(d, 1 \mathrm{H}, \mathrm{C}(\mathrm{O}) \mathrm{NH}, J=8.5 \mathrm{~Hz} ;{ }^{13} \mathrm{C} \mathrm{NMR}(125.76 \mathrm{MHz}\right.$, DMSO- $\left.d_{6}\right): \delta 51.7,55.6,66.6,110.6,110.7,111.6,111.7$, $122.8,123.5,123.8,124.0,124.1,126.0,126.6,126.9,129.3$, $131.3,131.9,142.5,144.4,144.6,148.9,149.0,151.6,151.7$, 163.9, 191.6, 191.8; IR (FTIR, $\mathrm{cm}^{-1}$ ): 982, 1024, 1122, 1140, 1163, 1265, 1333, 1514, 1593, 1655, 1670 and 3288; MS (ES, neg. mode) $\mathrm{m} / \mathrm{z}=622.2[\mathrm{M}-\mathrm{H}]^{-}$. 
4-((E)-1-Acrylamido-5-(3,4-dimethoxyphenyl)-2-((E)-3-(3,4dimethoxyphenyl)acryloyl)-3-oxopent-4-en-1-yl)benzoic acid (40)

$\mathrm{C}_{34} \mathrm{H}_{33} \mathrm{NO}_{9} ; \mathrm{M}=599.2$; yield: $63 \%$; m.p.: $218-220^{\circ} \mathrm{C} ;{ }^{1} \mathrm{H}$ NMR $\left(500.13 \mathrm{MHz}, \mathrm{DMSO}-d_{6}\right): \delta 3.76\left(s, 3 \mathrm{H}, \mathrm{OCH}_{3}\right), 3.78(s, 3 \mathrm{H}$, $\left.\mathrm{OCH}_{3}\right), 3.80\left(\mathrm{~s}, 3 \mathrm{H}, \mathrm{OCH}_{3}\right), 3.81\left(\mathrm{~s}, 3 \mathrm{H}, \mathrm{OCH}_{3}\right), 5.27(d, 1 \mathrm{H}$, $J=10.6 \mathrm{~Hz}, \mathrm{C}(\mathrm{O}) \mathrm{CHC}(\mathrm{O})), 5.54(d d$, overlapped peaks, $1 \mathrm{H}$, $J=10.2 \mathrm{~Hz}, \mathrm{CHH}=\mathrm{CH}), 5.88(t, 1 \mathrm{H}, J=9.5 \mathrm{~Hz}, \mathrm{CHNH}), 6.01(\mathrm{~d} d$, overlapped peaks, $1 \mathrm{H}, J=17.0 \mathrm{~Hz}, \mathrm{CH} H=\mathrm{CH}), 6.16(d d, 1 \mathrm{H}$, $J=6.8$ and $17.0 \mathrm{~Hz}, \mathrm{CHH}=\mathrm{CH}), 6.93-6.99(\mathrm{~m}, 2 \mathrm{H}), 6.99-7.06(\mathrm{~m}$, $2 \mathrm{H}), 7.21(d, 1 \mathrm{H}, J=8.3 \mathrm{~Hz}), 7.24(s, 1 \mathrm{H}), 7.31(d, 1 \mathrm{H}, J=8.3 \mathrm{~Hz})$, $7.35(s, 1 \mathrm{H}), 7.43(d, 1 \mathrm{H}, J=15.9 \mathrm{~Hz}), 7.53(d, 2 \mathrm{H}, J=8.3 \mathrm{~Hz})$, $7.71(d, 1 \mathrm{H}, J=15.9 \mathrm{~Hz}), 7.86(d, 1 \mathrm{H}, J=7.8 \mathrm{~Hz}), 8.71(d, 1 \mathrm{H}$, $J=8.9 \mathrm{~Hz}, \quad \mathrm{C}(\mathrm{O}) \mathrm{NH}), \quad 12.8$ (bs, $1 \mathrm{H}, \mathrm{C}(\mathrm{O}) \mathrm{OH}) ;{ }^{13} \mathrm{C} \mathrm{NMR}$ $\left(125.76 \mathrm{MHz}\right.$, DMSO- $d_{6}$ ): $\delta$ 51.7, $55.5,66.5,110.6,111.5$, $111.6,122.9,123.4,123.6,123.9,125.8,126.6,126.9,127.7$, $129.2,129.6,131.3,144.2,144.4,145.9,148.9,149.0,151.5$, 151.6, 163.7, 166.9, 191.5, 191.8; IR (FTIR, $\mathrm{cm}^{-1}$ ): 555, 800, 1024, 1131, 1261, 1512, 1593, 1716 and 2160; MS (ES, neg. mode) $m / z=598.2[\mathrm{M}-\mathrm{H}]^{-}$.

$N-((E)-5-(4-H y d r o x y-3-m e t h o x y p h e n y l)-2-((E)-3-(4-$ hydroxy-3-methoxyphenyl)acryloyl)-3-oxo-1-phenylpent4-en-1-yl)acrylamide (41)

$\mathrm{C}_{34} \mathrm{H}_{33} \mathrm{NO}_{9} ; \mathrm{M}=599.22$; yield: $63 \%$; m.p.: $218-220^{\circ} \mathrm{C} ;{ }^{1} \mathrm{H}$ NMR $\left(500.13 \mathrm{MHz}, \mathrm{DMSO}-d_{6}\right): \delta 3.76\left(s, 3 \mathrm{H}, \mathrm{OCH}_{3}\right), 3.78(s, 3 \mathrm{H}$, $\left.\mathrm{OCH}_{3}\right), 3.80\left(\mathrm{~s}, 3 \mathrm{H}, \mathrm{OCH}_{3}\right), 3.81\left(\mathrm{~s}, 3 \mathrm{H}, \mathrm{OCH}_{3}\right), 5.27(d, 1 \mathrm{H}$, $J=10.6 \mathrm{~Hz}, \mathrm{C}(\mathrm{O}) \mathrm{CHC}(\mathrm{O})), 5.54$ (dd, overlapped peaks, $1 \mathrm{H}$, $J=10.2 \mathrm{~Hz}, \mathrm{CHH}=\mathrm{CH}), 5.88(t, 1 \mathrm{H}, J=9.5 \mathrm{~Hz}, \mathrm{CHNH}), 6.01$ (dd, overlapped peaks, $1 \mathrm{H}, J=17.0 \mathrm{~Hz}, \mathrm{CHH}=\mathrm{CH}), 6.16(d d, 1 \mathrm{H}$, $J=6.8$ and $17.0 \mathrm{~Hz}, \mathrm{CHH}=\mathrm{CH}), 6.93-6.99(m, 2 \mathrm{H}), 6.99-7.06(m$, $2 \mathrm{H}), 7.21(d, 1 \mathrm{H}, J=8.3 \mathrm{~Hz}), 7.24(s, 1 \mathrm{H}), 7.31(d, 1 \mathrm{H}, J=8.3 \mathrm{~Hz})$, $7.35(s, 1 \mathrm{H}), 7.43(d, 1 \mathrm{H}, J=15.9 \mathrm{~Hz}), 7.53(d, 2 \mathrm{H}, J=8.3 \mathrm{~Hz})$, $7.71(d, 1 \mathrm{H}, J=15.9 \mathrm{~Hz}), 7.86(d, 1 \mathrm{H}, J=7.8 \mathrm{~Hz}), 8.71(d, 1 \mathrm{H}$, $J=8.9 \mathrm{~Hz}, \quad \mathrm{C}(\mathrm{O}) \mathrm{NH}), \quad 12.8$ (bs, $1 \mathrm{H}, \mathrm{C}(\mathrm{O}) \mathrm{OH}) ;{ }^{13} \mathrm{C} \quad \mathrm{NMR}$ $\left(125.76 \mathrm{MHz}\right.$, DMSO- $\left.d_{6}\right): \delta 51.7,55.5,66.5,110.6,111.5$, $111.6,122.9,123.4,123.6,123.9,125.8,126.6,126.9,127.7$, $129.2,129.6,131.3,144.2,144.4,145.9,148.9,149.0,151.5$, 151.6, 163.7, 166.9, 191.5, 191.8; IR (FTIR, $\mathrm{cm}^{-1}$ ): 978, 1028, 1122, 1165, 1207, 1269, 1514, 1589, 1662, 2359 and 3404; MS (ES, neg. mode) $\mathrm{m} / \mathrm{z}=598.2[\mathrm{M}-\mathrm{H}]^{-}$.

\section{4,4'-((1E,6E)-4-(Acrylamido(phenyl)methyl)-3,5-}

dioxohepta-1,6-diene-1,7-diyl)dibenzoic acid (42)

$\mathrm{C}_{31} \mathrm{H}_{25} \mathrm{NO}_{7} ; \mathrm{M}=523.16$; yield: $42 \%$; m.p.: $179-182^{\circ} \mathrm{C}^{1}{ }^{1} \mathrm{H}$ NMR $\left(500.13 \mathrm{MHz}, \mathrm{DMSO}-d_{6}\right): \delta 5.32(d, 1 \mathrm{H}, J=11.3 \mathrm{~Hz}), 5.50(d, 1 \mathrm{H}$, $J=11.2 \mathrm{~Hz}), 5.85(t, 1 \mathrm{H}, J=9.9 \mathrm{~Hz}), 5.99(d, 1 \mathrm{H}, J=16.5 \mathrm{~Hz})$, $6.14(d d, 1 \mathrm{H}, J=9.9$ and $16.5 \mathrm{~Hz}), 7.10-7.24(m, 3 \mathrm{H}), 7.25-7.33$ $(m, 3 \mathrm{H}), 7.42(d, 2 \mathrm{H}, J=7.24 \mathrm{~Hz}), 7.50(d, 1 \mathrm{H}, J=15.8 \mathrm{~Hz}), 7.72-$ $7.80(m, 2 \mathrm{H}), 7.84(d, 2 \mathrm{H}, J=7.9 \mathrm{~Hz}), 7.92(d, 2 \mathrm{H}, J=8.1 \mathrm{~Hz})$, $7.98(d, 2 \mathrm{H}, J=8.2 \mathrm{~Hz}), 8.69(d, 1 \mathrm{H}, J=8.9 \mathrm{~Hz}) ;{ }^{13} \mathrm{C} \mathrm{NMR}$ $\left(125.76 \mathrm{MHz}\right.$, DMSO- $\left.d_{6}\right)$ : $\delta 52.0,67.1,125.9,127.2,127.4$, $127.5,127.6,128.3,128.8,128.9,129.7,129.8,131.4,132.4$, 138.0, 138.2, 140.7, 142.3, 142.4, 163.7, 166.7, 166.8, 192.3, 192.6; IR (FTIR, $\mathrm{cm}^{-1}$ ): 985, 1084, 1173, 1288, 1410, 1533, 1610, 1659, 1693 and 3063; MS (ES, neg. mode) $\mathrm{m} / \mathrm{z}=522.2[\mathrm{M}-\mathrm{H}]^{-}$.
$N-((E)-5-(3,5-D i h y d r o x y p h e n y l)-2-((E)-3-(3,5-$ dihydroxyphenyl)acryloyl)-3-oxo-1-phenylpent-4-en-1-yl)acrylamide (43)

$\mathrm{C}_{29} \mathrm{H}_{25} \mathrm{NO}_{7} ; \mathrm{M}=499.16$; yield: $31 \%$; m.p.: $149^{\circ} \mathrm{C}$ (decomposed); ${ }^{1} \mathrm{H}$ NMR $\left(500.13 \mathrm{MHz}\right.$, DMSO- $\left.d_{6}\right): \delta 5.19(d, 1 \mathrm{H}$, $J=10.1 \mathrm{~Hz}, \mathrm{C}(\mathrm{O}) \mathrm{CHC}(\mathrm{O})), 5.53(d, 1 \mathrm{H}, J=9.0 \mathrm{~Hz}), 5.81(t, 1 \mathrm{H}$, $J=10.1 \mathrm{~Hz}, \mathrm{NHCH}), 6.00(d, 1 \mathrm{H}, J=15.8 \mathrm{~Hz}), 6.14(d d, 1 \mathrm{H}$, $J=10.1$ and $9.0 \mathrm{~Hz}), 6.20-6.61(m, 5 \mathrm{H}), 6.77(d, 1 \mathrm{H}, J=15.8 \mathrm{~Hz})$, $6.91(d, 1 \mathrm{H}, J=16.3 \mathrm{~Hz}), 7.10-7.54(m, 6 \mathrm{H}), 8.62(d, 1 \mathrm{H}$, $J=7.9 \mathrm{~Hz}), 9.34-9.55(4 \mathrm{H}, 4 \times \mathrm{OH}) ;{ }^{13} \mathrm{C} N M R(125.76 \mathrm{MHz}$, DMSO- $\left.d_{6}\right): \delta 51.9,67.1,105.5,106.7\left(2 \times \mathrm{CH}_{\mathrm{Ar}}\right), 124.7,124.8$, 125.8, 127.3, $127.5\left(2 \times \mathrm{CH}_{\text {Ar }}\right), 128.3\left(2 \times \mathrm{CH}_{\text {Ar }}\right), 131.4,135.5$, $135.8,140.8,144.4,144.5,158.6,158.7,163.6,192.2$ and 192.4; IR (FTIR, $\mathrm{cm}^{-1}$ ): 970, 1151, 1275, 1511, 1591, 1653, 2357 and 3375; MS (ES, neg. mode) $\mathrm{m} / \mathrm{z}=498.2[\mathrm{M}-\mathrm{H}]^{-}$.

N-((E)-5-(3-Hydroxyphenyl)-2-((E)-3-(3-hydroxyphenyl)acryloyl)-3-oxo-1-phenylpent-4-en-1-yl)-acrylamide (44) $\mathrm{C}_{29} \mathrm{H}_{25} \mathrm{NO}_{5} ; \mathrm{M}=467.17$; yield: $50 \%$; m.p.: $194-196^{\circ} \mathrm{C} ;{ }^{1} \mathrm{H} \mathrm{NMR}$ $\left(500.13 \mathrm{MHz}, \mathrm{DMSO}-d_{6}\right): \delta 5.22(d, 1 \mathrm{H}, J=10.6 \mathrm{~Hz}, \mathrm{C}(\mathrm{O}) \mathrm{CHC}$ $(\mathrm{O})), 5.52(d, 1 \mathrm{H}, J=11.1 \mathrm{~Hz}), 5.82(t, 1 \mathrm{H}, J=10.3 \mathrm{~Hz}, \mathrm{NHCH})$, $5.99(d, 1 \mathrm{H}, J=16.6 \mathrm{~Hz}), 6.13(d d, 1 \mathrm{H}, J=10.3 \mathrm{~Hz}), 6.83(d d, 2 \mathrm{H}$, $J=7.5$ and $8.0 \mathrm{~Hz}), 6.91(d, 1 \mathrm{H}, J=16.3 \mathrm{~Hz}), 6.96-7.44(m, 13 \mathrm{H})$, $7.60(d, 1 \mathrm{H}, J=16.3 \mathrm{~Hz}), 8.64(d, 1 \mathrm{H}, J=9.1 \mathrm{~Hz}), 9.61(s, 1 \mathrm{H}$, $\mathrm{OH}), 9.65(s, 1 \mathrm{H}, \mathrm{OH}) ;{ }^{13} \mathrm{C}$ NMR $\left(125.76 \mathrm{MHz}, \mathrm{DMSO}-d_{6}\right): \delta 51.9$, 67.1, 114.8, 114.9, $118.3\left(2 \times \mathrm{CH}_{\mathrm{Ar}}\right), 119.9\left(2 \times \mathrm{CH}_{\mathrm{Ar}}\right), 125.1$ $\left(2 \times \mathrm{CH}_{\mathrm{Ar}}\right), 125.8,127.3,127.6\left(2 \times \mathrm{CH}_{\mathrm{Ar}}\right), 128.3\left(2 \times \mathrm{CH}_{\mathrm{Ar}}\right)$, 130.0, 130.1, 131.5, 135.2, 135.5, 140.9, 144.0, 144.1, 157.7, 157.8, 163.7, 192.3 and 192.5; IR (FTIR, $\mathrm{cm}^{-1}$ ): 978, 1093, 1157, 1263, 1579, 1610, 1684, 2359 and 3290; MS (ES, neg. mode) $\mathrm{m} /$ $\mathrm{z}=466.2[\mathrm{M}-\mathrm{H}]^{-}$.

4-((E)-1-Acrylamido-5-(3,5-dihydroxyphenyl)-2-((E)-3-(3,5dihydroxyphenyl)acryloyl)-3-oxopent-4-en-1-yl)benzoic acid (45)

$\mathrm{C}_{30} \mathrm{H}_{25} \mathrm{NO}_{9}, \mathrm{M}=543.15$; yield: $34 \%$; m.p.: $122^{\circ} \mathrm{C}$ (decomposed); ${ }^{1} \mathrm{H}$ NMR $\left(500.13 \mathrm{MHz}, \mathrm{DMSO}-d_{6}\right)$ : $\delta 5.27(d, 1 \mathrm{H}, \mathrm{C}(\mathrm{O})$ $\mathrm{CHC}(\mathrm{O}), J=11.0 \mathrm{~Hz}), 5.54(d d, 1 \mathrm{H}, J=10.8$ and $1.7 \mathrm{~Hz}), 5.85(t$, $1 \mathrm{H}, J=9.5 \mathrm{~Hz}, \mathrm{NHCH}), 6.00(d, 1 \mathrm{H}, J=17.1 \mathrm{~Hz}), 6.15(d d, 1 \mathrm{H}$, $J=10.2$ and $10.5 \mathrm{~Hz}), 6.20-6.27(\mathrm{~m}, 1 \mathrm{H}), 6.30(\mathrm{bs}, 1 \mathrm{H}), 6.33$ (bs, $1 \mathrm{H}), 6.37(t, 1 \mathrm{H}, J=8.1 \mathrm{~Hz}), 6.41-6.45(m, 1 \mathrm{H}), 6.47(d, 2 \mathrm{H}$, $J=2.2 \mathrm{~Hz}), 6.55(d, 2 \mathrm{H}, J=2.2 \mathrm{~Hz}), 6.79(d, 1 \mathrm{H}, J=15.9 \mathrm{~Hz}), 6.91$ $(d, 1 \mathrm{H}, J=15.9 \mathrm{~Hz}), 7.28(d, 1 \mathrm{H}, J=15.9 \mathrm{~Hz}), 7.35-7.45(m, 1 \mathrm{H})$, 7.47-7.54 $(m, 3 \mathrm{H}), 7.85(d, 2 \mathrm{H}, J=8.5 \mathrm{~Hz}), 8.69(d, 1 \mathrm{H}$, $J=8.9 \mathrm{~Hz}), 9.53$ (bs, $1 \mathrm{H}, \mathrm{COOH}) ;{ }^{13} \mathrm{C} \mathrm{NMR}(125.76 \mathrm{MHz}$, DMSO- $\left.d_{6}\right): \delta 52.0,67.5,105.5,105.6,106.6,106.8\left(2 \times \mathrm{CH}_{\mathrm{Ar}}\right)$, 124.7, 124.8, 127.3, $127.6\left(2 \times \mathrm{CH}_{\mathrm{Ar}}\right), 128.2\left(2 \times \mathrm{CH}_{\mathrm{Ar}}\right), 135.6$, $135.9,141.1,144.3,144.4,158.6,158.7,158.8,168.2,192.4$ and 192.8; IR (FTIR, $\mathrm{cm}^{-1}$ ): 514, 1151, 1269, 1587, 1652, 1975, 2158 and 3238; MS (ES, neg. mode) $m / z=542.2[\mathrm{M}-\mathrm{H}]^{-}$.

$N-((E)-3-O x o-5-(4-0 x o-4 H-c h r o m e n-3-y l)-2-((E)-3-(4-0 x o-$ 4H-chromen-3-yl)acryloyl)-1-phenylpent-4-en-1-yl)acrylamide (46)

$\mathrm{C}_{35} \mathrm{H}_{25} \mathrm{NO}_{7} ; \mathrm{M}=571.16$; yield: $45 \%$; m.p.: $185-187^{\circ} \mathrm{C} ;{ }^{1} \mathrm{H}$ NMR $\left(500.13 \mathrm{MHz}\right.$, DMSO- $\left.d_{6}\right): \delta 5.05(d, 1 \mathrm{H}, J=11.2 \mathrm{~Hz}), 5.52(d, 1 \mathrm{H}$, 
$J=10.2 \mathrm{~Hz}), 5.81(t, 1 \mathrm{H}, J=10.2 \mathrm{~Hz}), 5.99(d, 1 \mathrm{H}, J=17.1 \mathrm{~Hz})$, $6.12(d d, 1 \mathrm{H}, J=10.0$ and $16.9 \mathrm{~Hz}), 7.17(t, 1 \mathrm{H}, J=7.4 \mathrm{~Hz}), 7.24-$ $7.30(m, 2 \mathrm{H}), 7.39(d, 2 \mathrm{H}, J=7.4 \mathrm{~Hz}), 7.48(d, 1 \mathrm{H}, J=15.6 \mathrm{~Hz})$, $7.53(d d, 3 \mathrm{H}, J=13.5$ and $6.9 \mathrm{~Hz}), 7.60(d, 1 \mathrm{H}, J=15.8 \mathrm{~Hz}), 7.66$ $(d, 1 \mathrm{H}, J=8.4 \mathrm{~Hz}), 7.69(d, 1 \mathrm{H}, J=8.4 \mathrm{~Hz}), 7.76-7.85(m, 3 \mathrm{H}), 8.1$ $(d, 1 \mathrm{H}, J=8.4 \mathrm{~Hz}), 8.12(d, 1 \mathrm{H}, J=7.4 \mathrm{~Hz}), 8.67(d, 1 \mathrm{H}$, $J=8.9 \mathrm{~Hz}), 8.83(s, 1 \mathrm{H}), 8.90(s, 1 \mathrm{H}) ;{ }^{13} \mathrm{C}$ NMR $(125.76 \mathrm{MHz}$, DMSO- $\left.d_{6}\right): \delta 52.0,68.6,117.8,118.1,118.5,123.4,123.5,125.4$, $125.5,125.7,126.2,126.8,127.3,127.6,128.2,131.5,134.7$, 136.1, 136.3, 140.8 155.0, 163.5, 175.1, 175.2, 192.3, 192.6; IR (FTIR, cm ${ }^{-1}$ ): 978, 1261, 1292, 1354, 1406, 1464, 1614, 1655, 2341 and 3350; MS (ES, neg. mode) $\mathrm{m} / \mathrm{z}=570.2[\mathrm{M}-\mathrm{H}]^{-}$.

$N-((E)-1-(4-F l u o r o p h e n y l)-5-(3-h y d r o x y p h e n y l)-2-((E)-3-(3-$ hydroxyphenyl)acryloyl)-3-oxopent-4-en-1-yl)acrylamide (47)

$\mathrm{C}_{29} \mathrm{H}_{24} \mathrm{FNO}_{7} ; \mathrm{M}=485.16$; yield: $53 \%$; m.p.: $200-201^{\circ} \mathrm{C} ;{ }^{1} \mathrm{H}$ NMR $\left(500.13 \mathrm{MHz}, \mathrm{DMSO}-d_{6}\right): \delta 5.22(d, 1 \mathrm{H}, J=10.9 \mathrm{~Hz}), 5.52(d$, $1 \mathrm{H}, J=11.6 \mathrm{~Hz}), 5.81(t, 1 \mathrm{H}, J=9.7 \mathrm{~Hz}), 5.99(d, 1 \mathrm{H}, J=17.4$ $\mathrm{Hz}), 6.13(d d, 1 \mathrm{H}, J=9.7$ and $16.8 \mathrm{~Hz}), 6.84(t, 2 \mathrm{H}, J=10.3 \mathrm{~Hz})$, $6.92(d, 1 \mathrm{H}, J=15.5 \mathrm{~Hz}), 7.00(s, 2 \mathrm{H}), 7.02-7.26(m, 7 \mathrm{H}), 7.37$ $(d, 1 \mathrm{H}, J=16.1 \mathrm{~Hz}), 7.40-7.47(m, 2 \mathrm{H}), 7.62(d, 1 \mathrm{H}, J=16.1 \mathrm{~Hz})$, $8.65(d, 1 \mathrm{H}, J=9.0 \mathrm{~Hz}), 9.62(s, 1 \mathrm{H}), 9.66(s, 1 \mathrm{H}) ;{ }^{13} \mathrm{C} N M R$ $\left(125.7 \mathrm{MHz}\right.$, DMSO- $\left.d_{6}\right): \delta 51.3,67.1,114.9,115.0,115.1$, $118.2,118.3,119.9,120.0,125.0,125.1,125.9,129.5,129.6$, 130.0, 130.1, 131.4, 135.1, 135.4, 137.1, 144.1, 144.2, 157.7, 157.8, 163.7, 192.1, 192.3; IR (FTIR, $\mathrm{cm}^{-1}$ ): 982, 1093, 1163, 1225, 1511, 1581, 1601, 1659, 1678 and 3286; MS (ES, neg. mode) $m / z=484.2[\mathrm{M}-\mathrm{H}]^{-}$.

$N-((4 E, 6 E)-7-(4-M e t h o x y p h e n y l)-2-((2 E, 4 E)-5-(4-$ methoxyphenyl)penta-2,4-dienoyl)-3-oxo-1-phenylhepta4,6-dienyl)acrylamide (48)

$\mathrm{C}_{35} \mathrm{H}_{33} \mathrm{NO}_{5} ; \mathrm{M}=547.24$; yield: $31 \%$; m.p.: $172-175^{\circ} \mathrm{C} ;{ }^{1} \mathrm{H}$ NMR $\left(500.13 \mathrm{MHz}, \mathrm{DMSO}-d_{6}\right): \delta 4.97(d, 1 \mathrm{H}, J=8.2 \mathrm{~Hz}), 5.52(d, 1 \mathrm{H}$, $J=6.7 \mathrm{~Hz}), 5.76(t, 1 \mathrm{H}, J=9.6 \mathrm{~Hz}), 5.94-6.17(m, 3 \mathrm{H}), 6.29-6.52$ $(m, 3 \mathrm{H}), 6.74-7.66(m, 23 \mathrm{H}), 8.60(s, 1 \mathrm{H}) ;{ }^{13} \mathrm{CNMR}(125.76 \mathrm{MHz}$, DMSO- $\left.d_{6}\right): \delta 52.8,56.2,68.3,115.3,125.3,125.5,126.5,127.6$, $127.8,128.1,128.4,129.0,129.37,129.43,129.8,130.0,132.4$, 141.8, 143.6, 143.7, 145.9, 146.0, 161.3, 164.4, 192.6, 192.9; IR (FTIR, $\mathrm{cm}^{-1}$ ): 999, 1028, 1174, 1254, 1510, 1578, 1660, 2359 and 3338; MS (ES, neg. mode) $\mathrm{m} / \mathrm{z}=546.2[\mathrm{M}-\mathrm{H}]^{-}$.

$N-((E)-5-(3-H y d r o x y p h e n y l)-2-((E)-3-(3-h y d r o x y p h e n y l)-$ acryloyl)-3-oxo-1-phenylpent-4-en-1-yl)cinnamamide (49) $\mathrm{C}_{35} \mathrm{H}_{29} \mathrm{NO}_{5} ; \mathrm{M}=543.20$; yield: $47 \%$; m.p.: $187-190^{\circ} \mathrm{C} ;{ }^{1} \mathrm{H}$ NMR $\left(500.13 \mathrm{MHz}, \mathrm{DMSO}-d_{6}\right): \delta 5.27(d, 1 \mathrm{H}, J=10.2 \mathrm{~Hz}, \mathrm{C}(\mathrm{O}) \mathrm{CHC}$ $(\mathrm{O})), 5.90(t, 1 \mathrm{H}, J=9.4 \mathrm{~Hz}), 6.56(d, 1 \mathrm{H}, J=15.5 \mathrm{~Hz}), 6.77-7.50$ $(m, 22 \mathrm{H}), 7.61(d, 1 \mathrm{H}, J=15.5 \mathrm{~Hz}), 8.68(d, 1 \mathrm{H}, J=9.0 \mathrm{~Hz}), 9.62$ $(s, 1 \mathrm{H}, \mathrm{OH}), 9.68(s, 1 \mathrm{H}, \mathrm{OH}) ;{ }^{13} \mathrm{C}$ NMR $\left(125.76 \mathrm{MHz}, \mathrm{DMSO}-d_{6}\right)$ : $\delta 52.0,67.1,114.8,114.9,118.2,118.3,119.9,121.9,125.1$, $125.2,127.3,127.5,127.6,128.2,128.8,129.5,130.0,130.1$, 134.7, 135.2, 135.4, 139.3, 140.9, 144.0, 144.1, 157.7, 157.8, 164.0, 192.3, 192.6; IR (FTIR, cm ${ }^{-1}$ ): 545, 696, 972, 1258, 1512, 1573, 1977, 2164 and 3280; MS (ES, neg. mode) $\mathrm{m} / \mathrm{z}=542.2$ $[\mathrm{M}-\mathrm{H}]^{-}$.
$N-((4 E, 6 E)-7-(4-H y d r o x y-3-m e t h o x y p h e n y l)-2-((2 E, 4 E)-5-$ (4-hydroxy-3-methoxyphenyl)penta-2,4-dienoyl)-3-oxo-1phenylhepta-4,6-dienyl)cinnamamide (50)

$\mathrm{C}_{41} \mathrm{H}_{37} \mathrm{NO}_{7} ; \mathrm{M}=655.26$; yield: $36 \%$; m.p.: $128-131^{\circ} \mathrm{C} ;{ }^{1} \mathrm{H}$ NMR $\left(500.13 \mathrm{MHz}\right.$, DMSO- $\left.d_{6}\right): \delta 3.79(s, 3 \mathrm{H}), 3.80(s, 3 \mathrm{H}), 4,97(d, 1 \mathrm{H}$, $J=10.9 \mathrm{~Hz}), 5.83(t, 1 \mathrm{H}, J=9.9 \mathrm{~Hz}), 6.34(d, 2 \mathrm{H}, J=14.9 \mathrm{~Hz})$, $6.47(d, 2 \mathrm{H}, J=15.2 \mathrm{~Hz}), 6.54(d, 2 \mathrm{H}, J=15.9 \mathrm{~Hz}), 6.74-6.79(m$, $3 \mathrm{H}), 6.87-7.54(m, 17 \mathrm{H}), 8.64(d, 1 \mathrm{H}, J=8.9 \mathrm{~Hz}), 9.50(s, 1 \mathrm{H})$, $9.51(s, 1 \mathrm{H}) ;{ }^{13} \mathrm{C}$ NMR $\left(125.76 \mathrm{MHz}, \mathrm{DMSO}-d_{6}\right): \delta 52.9,56.47$, $56.49,68.4,111.2,116.5,122.8,123.10,123.14,124.7,124.8$, $127.0,127.3,128.1,128.4,129.1,129.7,130.4,135.6,140.1$, $141.9,144.5,144.6,146.1,146.2,148.8,149.5,149.5,164.8$, 192.7, 193.0; IR (FTIR, $\mathrm{cm}^{-1}$ ): 997, 1034, 1165, 1282, 1514, 1564, 1660, 2355 and 3327; MS (ES, neg. mode) $\mathrm{m} / \mathrm{z}=654.2[\mathrm{M}-\mathrm{H}]^{-}$.

$N-((E)-5-(3,5-D i h y d r o x y p h e n y l)-2-((E)-3-(3,5-$ dihydroxyphenyl)acryloyl)-3-oxo-1-phenylpent-4-en-1-yl)cinnamamide (51)

$\mathrm{C}_{35} \mathrm{H}_{29} \mathrm{NO}_{7} ; \mathrm{M}=575.19$; yield: $52 \%$; m.p.: $206-209^{\circ} \mathrm{C} ;{ }^{1} \mathrm{H}$ NMR $\left(500.13 \mathrm{MHz}, \mathrm{DMSO}-d_{6}\right): \delta 5.23(d, 1 \mathrm{H}, J=10.8 \mathrm{~Hz}), 5.87(t, 1 \mathrm{H}$, $J=9.7 \mathrm{~Hz}), 6.31(d, 2 \mathrm{H}, J=8.2 \mathrm{~Hz}), 6.47(s, 2 \mathrm{H}), 6.54(s, 2 \mathrm{H}), 6.81$ $(d, 1 \mathrm{H}, J=16.1 \mathrm{~Hz}), 6.93(d, 1 \mathrm{H}, J=16.1 \mathrm{~Hz}), 7.18(t, 1 \mathrm{H}$, $J=7.1 \mathrm{~Hz}), 7.24-7.38(m, 8 \mathrm{H}), 7.42(d, 2 \mathrm{H}, J=6.4 \mathrm{~Hz}), 7.45-7.52$ $(m, 3 \mathrm{H}), 8.66(d, 1 \mathrm{H}, J=8.9 \mathrm{~Hz}), 9.46(s, 2 \mathrm{H}), 9.48(s, 2 \mathrm{H}))$; ${ }^{13} \mathrm{C}$ NMR $\left(125.76 \mathrm{MHz}\right.$, DMSO- $\left.d_{6}\right): \delta 52.0,67.1,105.4,105.5$, $106.7,121.9,124.8,124.9,127.3,127.5,127.6,128.2,128.9$, $129.5,134.7,135.5,135.8,139.3,140.9,144.4,144.5,158.7$, 158.8, 164.0, 192.3, 192.6; IR (FTIR, $\mathrm{cm}^{-1}$ ): 974, 1088, 1159, 1271, 1350, 1452, 1506, 1601, 1606, 2353 and 3263; MS (ES, neg. mode) $m / z=574.2[\mathrm{M}-\mathrm{H}]^{-}$.

$N-((E)-5-(3,4-D i m e t h o x y p h e n y l)-2-((E)-3-(3,4-$ dimethoxyphenyl)acryloyl)-3-oxo-1-phenylpent-4-en-1-yl)cinnamamide (52)

$\mathrm{C}_{39} \mathrm{H}_{37} \mathrm{NO}_{7} ; \mathrm{M}=631.26$; yield: $51 \%$; m.p.: $212-215^{\circ} \mathrm{C} ;{ }^{1} \mathrm{H}$ NMR $\left(500.13 \mathrm{MHz}\right.$, DMSO- $\left.d_{6}\right): \delta 3.73-3.82(m, 12 \mathrm{H}), 5.24(d, 1 \mathrm{H}$, $J=10.5 \mathrm{~Hz}), 5.90(t, 1 \mathrm{H}, J=9.5 \mathrm{~Hz}), 6.56(d, 1 \mathrm{H}, J=15.2 \mathrm{~Hz})$, 6.90-7.08 $(m, 4 \mathrm{H}), 7.14-7.39(m, 11 \mathrm{H}), 7.39-7.52(m, 5 \mathrm{H}), 7.68$ $(d, 1 \mathrm{H}, J=16.2 \mathrm{~Hz}), 8.67(d, 1 \mathrm{H}, J=9.5 \mathrm{~Hz}) ;{ }^{13} \mathrm{C} \quad$ NMR $\left(125.76 \mathrm{MHz}\right.$, DMSO- $\left.d_{6}\right): \delta 52.0,55.6,66.9,110.6,111.6,111.7$, $122.0,123.1,123.5,123.6,123.9,126.7,127.0,127.2,127.5$, $127.6,128.2,128.9,129.5,134.7,139.2,141.2,144.1,144.2$, 148.9, 149.0, 151.5, 151.6, 163.2, 191.9, 192.3; IR (FTIR, $\mathrm{cm}^{-1}$ ): 984, 1020, 1138, 1263, 1516, 1591, 1659, 1676, 2337 and 3415; MS (ES, neg. mode) $\mathrm{m} / \mathrm{z}=630.2[\mathrm{M}-\mathrm{H}]^{-}$.

\section{Biological evaluation}

Cell toxicity measurements with novel curcumin analogues A549 and H1975 lung adenocarcinoma cell lines were maintained in DMEM supplemented with $10 \%$ FBS. Cell toxicity measurements were carried out in 96-well microtitre plates with 6000 cells seeded in each well. Test measurements were carried out in duplicate, and control measurements in quadruplicate. After an overnight incubation, cells were treated with an increasing concentration of the analogue. At $72 \mathrm{~h}$ post-treatment, the cell viability was measured by means 
of the homogeneous, colourimetric MTS assay (Promega). Cell viability of treated cells was calculated relative to that of DMSO vehicle controls. $\mathrm{Gl}_{50}$ values were calculated using GraphPad Prism 5 software, non-linear regression fitting with variable slope was applied.

\section{Holographic cell analysis}

Digital holographic images were taken with the HoloMonitor M3 instrument (Phase Holographic Imaging $A B$, Phiab, Sweden). Cells of lung cancer line A549 were treated with either $25 \mu \mathrm{M} 1$ or $0.5 \mu \mathrm{M} 44$ in T25 flasks and incubated for $24 \mathrm{~h}$. Holographic images of the same culture area were captured before and after treatment. Time-matched untreated cells were imaged as controls. Cell morphological changes were analysed with Holostudio 2.4 software (Phase Holographic Imaging $A B$, Phiab, Sweden). Each point in the scatterplot represents data on a single cell. Parameters reflecting changes in cell morphology (e.g. area, thickness, roughness, eccentricity, irregularity and volume) were recorded [37].

\section{$N F-\kappa B$ activation assay}

The B16 cell line was maintained in RPMI medium (Lonza) supplemented with $10 \%$ FCS (Lonza). The NF- $\mathrm{BB}$ reporter cell lines were created by transfection with the pNF-кB-Luc/neo reporter construct with the Lipofectamine 2000 reagent (Invitrogen). Stable cell lines were selected by G418 (Sigma) treatment. One-day-old cultures of B16/NF-кB-Luc cells, grown on luminoplates (Corning-Costar) were used. Cells $\left(5 \times 10^{4}\right.$ cells/well) were induced with 100 units $/ \mathrm{mL}$ TNF-alpha. Each measurement was carried out in duplicate. After $6 \mathrm{~h}$ of incubation with inducers, the medium was removed, and cells were washed and lysed for $10 \mathrm{~min}$ at room temperature in Cell Culture Lysis Reagent ( $20 \mu \mathrm{L} /$ well; Promega). Substrate was then added ( $20 \mu \mathrm{L} /$ well; Promega), and luciferase activity was measured in a Luminoskan Ascent (Thermo Electron Corporation) scanning luminometer. Cell viability was routinely determined through use of the Trypan blue exclusion test during the assays to make sure that assays were always carried out on viable cells. A total of $80 \%$ of all measurements displayed a variation of less than $20 \%$. IC $C_{50}$ values were calculated using GraphPad Prism 5 software, non-linear regression fitting with variable slope was applied.

\section{UPR-related gene expression analysis}

Total RNA was purified from $4 \times 10^{5}$ 1-treated, analoguetreated or control ( $0.2 \%$ DMSO) cells $(6 \mathrm{~h}$ after treatment) with the AccuZol ${ }^{\mathrm{TM}}$ RNA purification kit (Bioneer, Daeleon, Korea) according to the manufacturer's protocol. All treatments and controls involved three biological replicates. Total RNA $(2 \mu \mathrm{g})$ was converted into cDNA with the High-Capacity cDNA Archive Kit (Applied Biosystems, Foster City, CA, USA) in a total volume of $20 \mu \mathrm{L}$; and without purification, the mixture was diluted fivefold, and $2 \mu \mathrm{L}$ was subjected to QRT-PCR analysis. QRT-PCR was performed on the Exicycler instrument (Bioneer) with gene-specific primers (heat shock protein family A (Hsp70) member 5, HSPA5_F: 5'-agcagggccttcaccaat3'; HSPA5_R: 5'-cgtcgcctactcggcttat-3'; DNA damage inducible transcript 3, DDIT3_F: 5'-cagagctggaacctgaggag-3'; DDIT3_R: 5'-tggatcagtctggaaaagca-3'; activating transcription factor 4, ATF4_F: 5'-tctccagcgacaaggctaa-3'; ATF4_R: 5'-caatctgtcccggagaagg-3'; X-box binding protein 1, XBP1_F: 5'-ggagttaagacagcgcttgg-3'; ATF4_F: 5'-tctccagcgacaaggctaa-3'; ATF4_R: 5'-caatctgtcccggagaagg- $3^{\prime} ;$ XBP1_R: 5'-cactggcctcacttcattcc-3'; actin beta, ACTB_F: 5'-attggcaatgagcggttc-3'; ACTB_R: 5'cgtggatgccacaggact- $3^{\prime}$ ) and the SybrGreen protocol [45]. Curves were analysed by using dynamic tube and slope correction methods, data from cycles close to the baseline being ignored. Relative expression ratios were normalized to beta-actin. Statistical analysis: Student's t-test was performed in Excel.

\section{Autophagy-related gene expression analysis}

Total RNA was purified from $2 \times 10^{5}$ A549 cells. Three biological replicates were applied for all conditions. Treatments included: $500 \mathrm{nM}$ rapamycin, $0.5,1$ and $5 \mu \mathrm{M} 44$ and untreated controls. Samples were collected after 6, 12 and $24 \mathrm{~h}$ after treatments ( $6 \mathrm{~h}$ after treatment) with the AccuZol ${ }^{\mathrm{TM}}$ RNA purification kit (Bioneer, Daeleon, Korea) according to the manufacturer's protocol. All treatments and controls involved three biological replicates. Total RNA $(1.5 \mu \mathrm{g})$ was converted into CDNA with the High-Capacity CDNA Archive Kit (Applied Biosystems, Foster City, CA, USA) in a total volume of $20 \mu \mathrm{L}$; and without purification, the mixture was diluted fivefold, and $2 \mu \mathrm{L}$ was subjected to QRT-PCR analysis. QRT-PCR was performed on the Light Cycler Nano Instrument (Roche) with gene-specific primers (autophagy related 5, ATG5_F: 5'tggatttcgttatatccctttag-3'; ATG5_R: 5'-cctagtgtgtgcaactg tcca-3'; beclin 1, BECN1_F: 5'-ggatggtgtctctcgcagat-3'; BECN1_R: 5'-ttggcactttctgtggacat-3'; GABA type A receptorassociated protein, GABARAP_F: 5'-ggtgccttctgatctcacagt-3'; GABARAP_R: 5'-tgggtggaatgacattgttg-3'; microtubule associated protein 1 light chain 3 beta, MAP1LC3B_F: $5^{\prime}$-cgcaccttcgaacaaagag-3'; MAP1LC3B_R: 5'-ctcaccttgtatcgttctattatca-3'; sequestosome 1, SQSTM1_F: 5'-agctgccttgtacccacatc-3'; SQSTM1_R: 5'-cagagaagcccatggacag-3') and the SybrGreen protocol [45]. Relative expression ratios were normalised to beta-actin. Statistical analysis: Student's t-test was performed in Excel.

\section{Western blot analysis}

Western blot samples were obtained by scraping $1 \times 10^{6} \mathrm{~A} 549$ cells from 6 -well plates in $200 \mu \mathrm{L}$ of hot Laemmli buffer. A total of $20 \mu \mathrm{L}$ samples were run on a $20 \%$ SDS-PAGE and blotted onto Immobilon P PVDF membrane (Millipore, $0.45 \mu \mathrm{m}$ pore size). After blocking with $0.5 \%$ non-fat dry milk powder in PBS containing $0.05 \%$ Tween 20, membranes were labeled 1:175 with MAP1LC3B antibody (ABfinity ${ }^{\mathrm{TM}}$ rabbit monoclonal, ThermoFisher Scientific, \#700712). Proteins were visualised using the ECL system (Luminata Forte Western HRP substrate, Millipore, \#WBLUF0100). Quantification was carried out using ImageJ software by referring the 
relative density of $\mathrm{LC} 3 \mathrm{~B}$ bands to the untreated sample relative density. Data are shown adjusted relative density of these ratios. For loading control Ponceau staining was applied [46].

\section{Spinning disk confocal microscopy}

HeLa cells stably expressing the GFP-mRFP-LC3 construct were seeded onto poly-D-lysine (Sigma) treated $19 \mathrm{~mm}$ cover glass (Menzel-Glaser) in 6-well plates at a density of 15000 cells/well and grown overnight. They were treated with $500 \mathrm{nM}$ rapamycin (AK Scientific), $100 \mathrm{nM}$ bafilomycin A1 (Sigma) or $0.5,1$ and $5 \mu \mathrm{M}$ 44. After $6 \mathrm{~h}$ incubation, images were recorded using Visitron spinning disk confocal system having Yokogawa CSU-W1 spinning disk unit (pinhole diameter, $50 \mu \mathrm{m})$, Andor Zyla 4.2 Plus camera and Olympus IX83 inverted microscope (Visitron systems $\mathrm{GmBH}$, Germany). Images of multiple fields of each coverglass were recorded in multiwell dishes using LUCPlan FL $40 \times$ long working distance objective with correction collar (numerical aperture 0.6) and at $1024 \times 1024$ resolution. GFP images were captured using $700 \mathrm{~ms}$ camera exposure and $30 \% 488 \mathrm{~nm}$ laser. For mRFP imaging, $500 \mathrm{~ms}$ camera exposure and $60 \% 561 \mathrm{~nm}$ laser were used. Images were psudocolored as green (GFP) and red (mRFP). Images were prepared by using Adobe Photoshop software.

\section{Liposome encapsulation of compound 44}

Liquid-phase 44-containing liposomes were prepared by dissolving lipid powder CHOL/PC/DSPE-mPEG (62/28/0.6 mol $\%)$ and $44(9.2 \mathrm{~mol} \%)$ in EtOH. After the solvent had been removed, the lipid film was hydrated and redispersed in PBS solution to a final 44 concentration of $1 \mathrm{mg} / \mathrm{mL}$. The dispersion was subjected to size extrusion $(0.45 \mu \mathrm{m}$ pore size) and finally filtered through sterile filters $(0.22 \mu \mathrm{m}$ pore size $)$.

In vivo effects of $\mathbf{4 4}$ on lung cancer xenografts

Male SCID mice ( 6 weeks old, 22-24 g body weight) supplied by Innovo Ltd. (Budapest, Hungary) were housed in sterile cages. They were fed autoclaved food and sterile water ad libitum. For inoculation into SCID mice, the A549 cells were trypsinised, washed and resuspended in sterile PBS. With this suspension $\left(6 \times 10^{6}\right.$ cells in $\left.0.2 \mathrm{~mL}\right)$, the mice were injected subcutaneously in the dorsal region, unilaterally. All operative procedures and animal care conformed strictly to the Hungarian Council on Animal Care guidelines. The administration of 44-containing liposomes into the tail veins of SCID mice started on day 4 and was continued three times a week for 4 weeks in a dose of $3 \mathrm{mg} / \mathrm{kg}$. The tumour size was measured with calipers every 2-3 days and the volume was calculated via the formula $L \times W \times H$, where $L$ was the length, $W$ the width and $H$ the height of the tumour.

This work was supported by the following grant: GOP-1.1.111-2011-0003.

The authors have declared no conflicts of interest.

\section{References}

[1] P. Fresco, F. Borges, M. P. Marques, C. Diniz, Curr. Pharm. Des. 2010, 16, 114-134.

[2] B. B. Aggarwal, A. Kumar, A. C. Bharti, Anticancer Res. 2003, 23, 363-398.

[3] B. B. Aggarwal, B. Sung, Trends Pharmacol. Sci. 2009, 30, 85-94.

[4] H. Zhou, C. S. Beevers, S. Huang, Curr. Drug. Targets 2011, 12, 332-347.

[5] S. Singh, A. Khar, Anticancer Agents Med. Chem. 2006, 6, 259-270.

[6] F. H. Sarkar, Y. Li, Z. Wang, D. Kong, Cell. Signal. 2009, 21, 1541-1547.

[7] Z. Wang, Y. Zhang, S. Banerjee, Y. Li, F. H. Sarkar, Cancer 2006, 106, 2503-2513.

[8] S. Singh, B. B. Aggarwal, J. Biol. Chem. 1995, 270, 24995-25000.

[9] T. Leu, M. Maa, Curr. Med. Chem.: Anti-Cancer Agents 2002, 2, 357-370.

[10] V. Milacic, S. Banerjee, K. Landis-Piwowar, F. Sarkar, A. Majumdar, Q. Dou, Cancer Res. 2008, 68, 7283-7292.

[11] J. M. Holy, Mutat. Res. 2002, 518, 71-84.

[12] K. Wolanin, A. Magalska, G. Mosieniak, R. Klinger, S. McKenna, S. Vejda, E. Sikora, K. Piwocka, Mol. Cancer Res. 2006, 4, 457-469.

[13] H. Aoki, Y. Takada, S. Kondo, R. Sawaya, B. B. Aggarwal, Y. Kondo, Mol. Pharmacol. 2007, 72, 29-39.

[14] G. O'Sullivan-Coyne, G. C. O'Sullivan, T. R. O'Donovan, K. Piwocka, S. L. McKenna, Br. J. Cancer 2009, 101, 1585-1595.

[15] M. X. Ye, Y. Li, H. Yin, J. Zhang, Int. J. Mol. Sci. 2012, 13, 3959-3978.

[16] Y. J. Lee, N. Y. Kim, Y. A. Suh, C. Lee, Korean J. Physiol. Pharmacol. 2011, 15, 1-7.

[17] P. Anand, A. B. Kunnumakkara, R. A. Newman, B. B. Aggarwal, Mol. Pharm. 2007, 4, 807-818.

[18] H. Hatcher, R. Planalp, J. Cho, F. M. Torti, S. V. Torti, Cell Mol. Life Sci. 2008, 65, 1631-1652.

[19] B. K. Adams, E. M. Ferstl, M. C. Davis, M. Herold, S. Kurtkaya, R. F. Camalier, M. G. Hollingshead, G. Kaur, E. A. Sausville, F. R. Rickles, J. P. Snyder, D. C. Liotta, M. Shoji, Bioorg. Med. Chem. 2004, 12, 3871-3883.

[20] D. Simoni, M. Rizzi, R. Rondanin, R. Baruchello, P. Marchetti, F. P. Invidiata, M. Labbozzetta, P. Poma, V. Carina, M. Notarbartolo, A. Alaimo, N. D'Alessandro, Bioorg. Med. Chem. Lett. 2008, 18, 845-849.

[21] J. R. Fuchs, B. Pandit, D. Bhasin, J. P. Etter, N. Regan, D. Abdelhamid, C. Li, J. Lin, P. Li, Bioorg. Med. Chem. Lett. 2009, 19, 2065-2069.

[22] A. Katsori, M. Chatzopoulou, K. Dimas, C. Kontogiorgis, A. Patsilinakos, T. Trangas, D. Hadjipavlou-Litina, Eur. J. Med. Chem. 2011, 46, 2722-2735.

[23] P. Lagisetty, P. Vilekar, K. Sahoo, S. Anant, V. Awasthi, Bioorg. Med. Chem. 2010, 18, 6109-6120. 
[24] G. Liang, L. Shao, Y. Wang, C. Zhao, Y. Chu, J. Xiao, Y. Zhao, X. Li, S. Yang, Bioorg. Med. Chem. 2009, 17, 2623-2631.

[25] L. Lin, Q. Shi, A. K. Nyarko, K. F. Bastow, C. C. Wu, C. Y. Su, C. C. Shih, K. H. Lee, J. Med. Chem. 2006, 49, 3963-3972.

[26] G. Z. Zhou, S. N. Zhang, L. Zhang, G. C. Sun, X. B. Chen, Pharm. Biol. 2014, 52, 111-116.

[27] G. Z. Zhou, S. L. Xu, G. C. Sun, X. B. Chen, Mol. Med. Rep. 2014, 10, 441-446.

[28] G. Z. Zhou, G. C. Sun, S. N. J. Zhang, Biochem. Mol. Toxicol. 2015, 29, 267-273.

[29] Y. Q. Xia, X. Y. Wei, W. L. Li, K. Kanchana, C. C. Xu, D. H. nChen, P. H. Chou, R. Jin, J. Z. Wu, G. Liang, Asian Pac. J. Cancer Prev. 2014, 15, 6893-6898.

[30] X. Qiu, Y. Du, B. Lou, Y. Zuo, W. Shao, Y. Huo, J. Huang, Y. Yu, B. Zhou, J. Du, H. Fu, X. Bu, J. Med. Chem. 2010, 53, 8260-8273.

[31] C. Chen, Y. Liu, Y. Chen, J. Xu, Cancer. Biol. Ther. 2011, 12, 797-807.

[32] M. W. Amolins, L. B. Peterson, B. S. J. Blagg, Bioorg. Med. Chem. 2009, 17, 360-367.

[33] H. Mao, J. Wan, Y. Pan, Tetrahedron 2009, 65, 1026-1032.

[34] K. Zumbansen, A. Döhring, B. List, Adv. Synth. Catal. 2010, 352, 1135-1138.

[35] S. Venkateswarlu, M. S. Ramachandra, G. V. Subbaraju, Bioorg. Med. Chem. 2005, 13, 6374-6380.

[36] A. Mölder, M. Sebesta, M. Gustafsson, L. Gisselson, A. G. Wingren, K. Alm, J. Microsc. 2008, 232, 240-247.
[37] R. Madácsi, I. Kanizsai, L. Z. Fehér, M. Gyuris, B. Ozsvári, A. Erdélyi, J. Wölfling, L. G. Puskás, Med. Chem. 2013, 9, 911-919.

[38] B. Hoesel, J. A. Schmid, Mol. Cancer 2013, 12, 86.

[39] A. C. Bharti, N. Donato, S. Singh, B. B. Aggarwal, Blood 2003, 101, 1053-1062.

[40] S. Bernales, F. R. Papa, P. Walter, Annu. Rev. Cell. Dev. Biol. 2006, 22, 487-508.

[41] M. Zheng, Q. Zhang, Y. Joe, B. H. Lee, D. G. Ryu, K. B. Kwon, S. W. Ryter, H. T. Chung, Int. Immunopharmacol. 2013, 15, 517-523.

[42] V. Basile, S. Belluti, E. Ferrari, C. Gozzoli, S. Ganassi, D. Quaglino, M. Saladini, C. Imbriano, PLoS ONE 2013, 8, e53664.

[43] J. X. Song, Y. R. Sun, I. Peluso, Y. Zeng, X. Yu, J. H. Lu, Z. Xu, M. Z. Wang, L. F. Liu, Y. Y. Huang, L. L. Chen, S. S. Durairajan, H. J. Zhang, B. Zhou, H. Q. Zhang, A. Lu, A. Ballabio, D. L. Medina, Z. Guo, M. Li, Autophagy 2016, 12(8), 1372-1389.

[44] C. Settembre, C. Di Malta, V. A. Polito, M. G. Arencibia, F. Vetrini, S. Erdin, S. U. Erdin, T. Huynh, D. Medina, P. Colella, M. Sardiello, D. C. Rubinsztein, A. Ballabio, Science 2011, 332, 1429-1433.

[45] A. Catalá, A. Zvara, L. G. Puskás, K. Kitajka, J. Pineal. Res. 2007, 42, 43-49.

[46] I. Romero-Calvo, B. Ocón, P. Martínez-Moya, M. D. Suárez, A. Zarzuelo, O. Martínez-Augustin, F. S. de Medina, Anal. Biochem. 2010, 401, 318-320. 